Aus der Klinik für Unfallchirurgie und Orthopädie - Abteilung Unfallchirurgie, Plastischeund Wiederherstellungschirurgie

(Prof. Dr. med. K. M. Stürmer)

im Zentrum Chirurgie

der Medizinischen Fakultät der Universität Göttingen

\title{
Relevanz des Musculus vastus medialis obliquus als Prädispositionsfaktor für die Entstehung der akuten und chronischen Patellaluxation
}

\author{
INAUGURAL-DISSERTATION \\ zur Erlangung des Doktorgrades \\ der Medizinischen Fakultät der \\ Georg-August-Universität zu Göttingen
}

vorgelegt von

Swantje Oberthür

aus

Wilhelmshaven

Göttingen 2015 
Dekan:

I. Berichterstatter: PD Dr. med. P. Balcarek

II. Berichterstatter/in:

III. Berichterstatter/in:

Tag der mündlichen Prüfung:
Prof. Dr. rer. nat. H. K. Kroemer 


\section{Inhaltsverzeichnis}

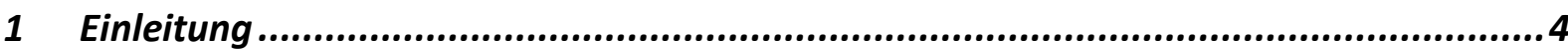

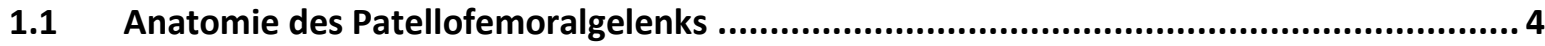

1.1.1 Knöcherne Strukturen des Patellofemoralgelenks ........................................................................ 4

1.1.2 Ligamentäre Strukturen des Patellofemoralgelenks ............................................................... 5

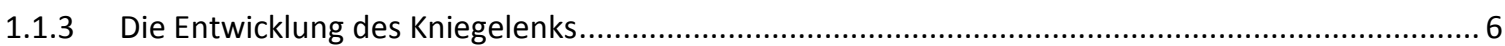

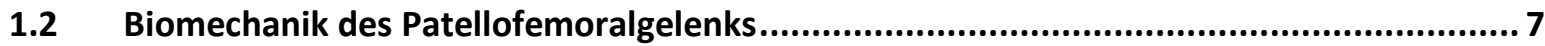

1.2.1 Patellofemoraler Bewegungsablauf und die besondere Bedeutung der Patella ............................. 7

1.2.2 Die Komponenten der Stabilität des Patellofemoralgelenks......................................................... 8

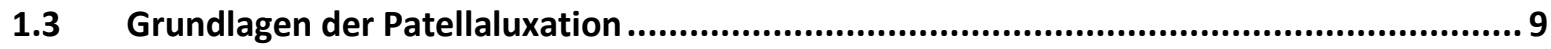

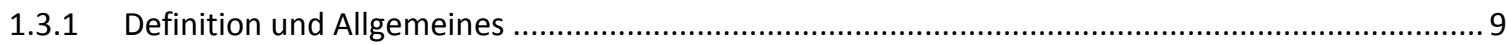

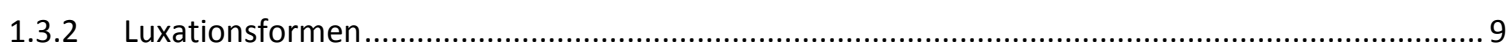

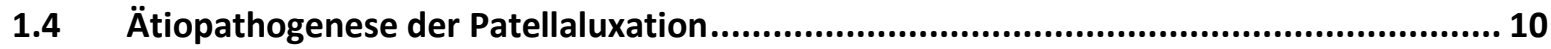

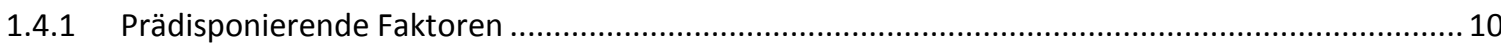

1.5 Der M. vastus medialis obliquus und seine Funktion im Patellofemoralgelenk ............... 13

1.5.1 Anatomie und Biomechanik des VMO und seine Bedeutung für die Stabilität des PFG ...............13

1.5.2 Der VMO im Kontext des Krankheitsbildes der Patellaluxation und des patellofemoralen

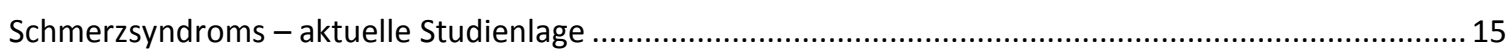

1.5.3 Prinzipien der konservativen Patellaerstluxationstherapie und die Rolle des VMO ...................... 17

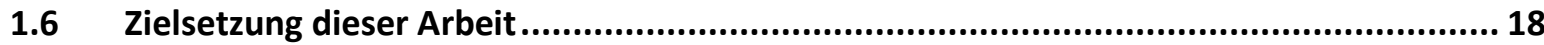

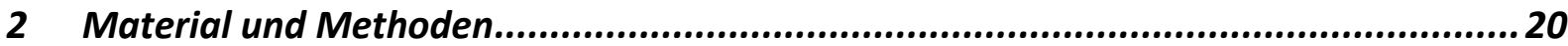

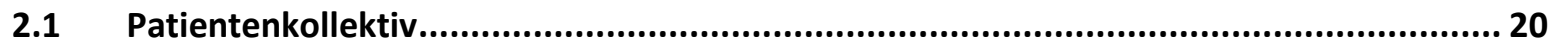

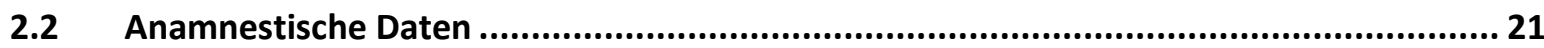

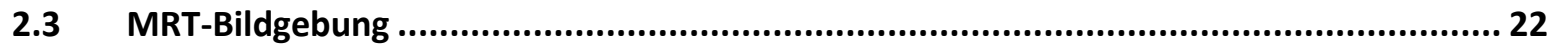

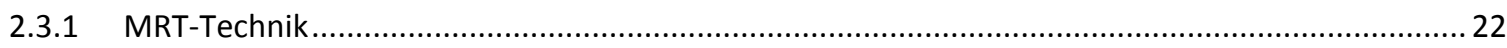

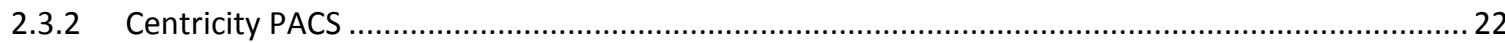

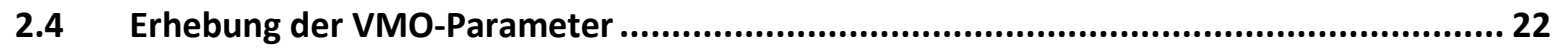

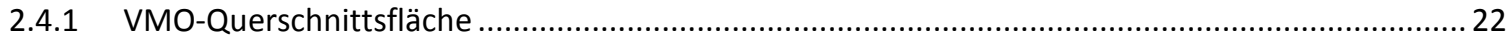

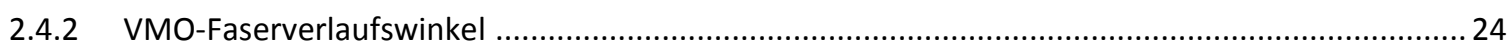

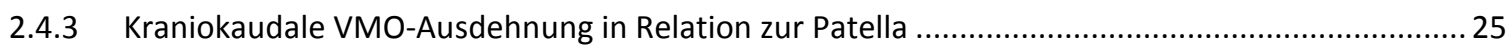

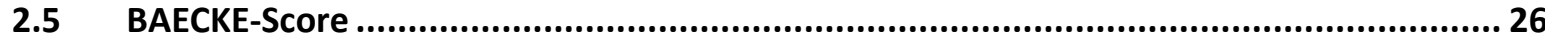

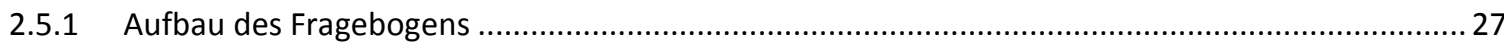




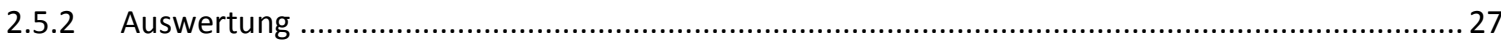

2.6 Erhebung der anatomischen Hauptparameter der Patellaluxation................................ 28

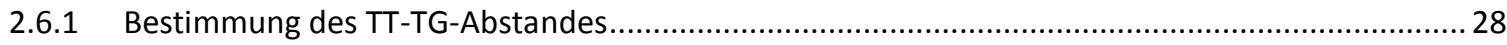

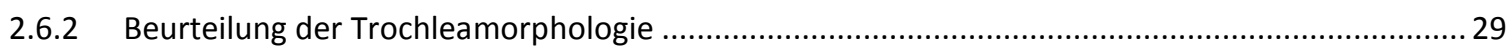

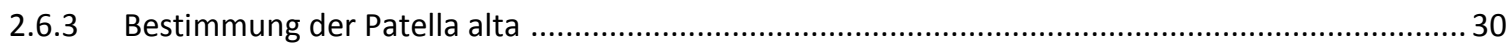

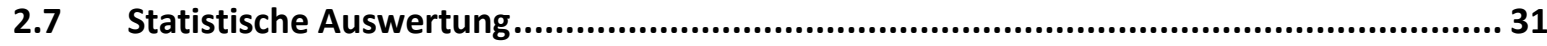

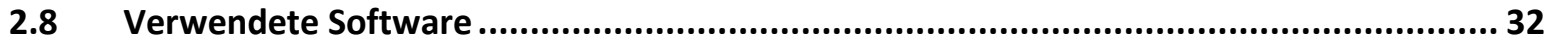

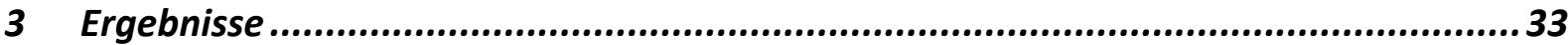

3.1 Demographische Daten des Patientenkollektivs.......................................................... 33

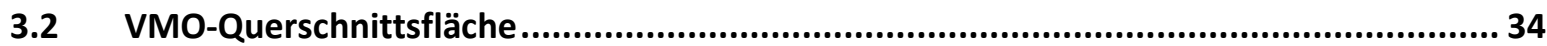

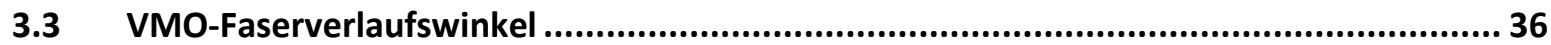

3.4 Kraniokaudale VMO-Ausdehnung in Relation zur Patella ............................................. 37

3.5 Anatomische Hauptparameter der Patellaluxation....................................................... 38

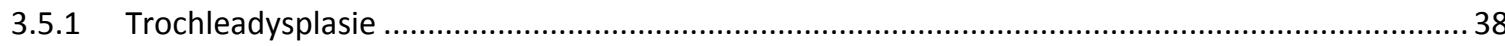

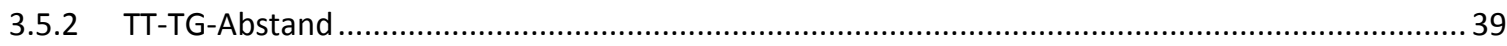

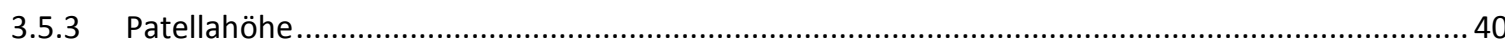

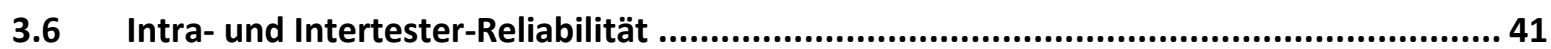

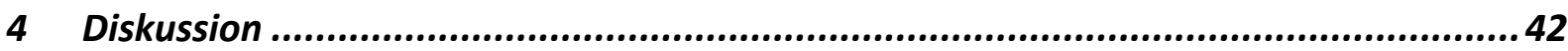

4.1 Demographische Daten des Patientenkollektivs......................................................... 42

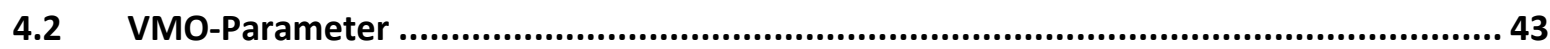

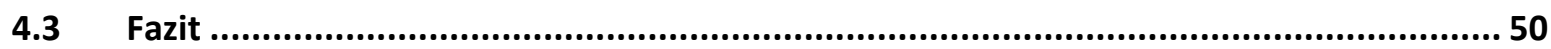

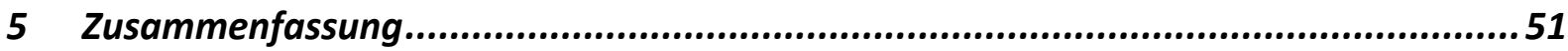

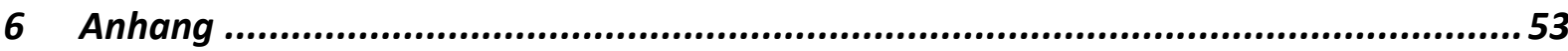

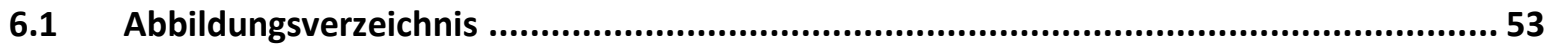

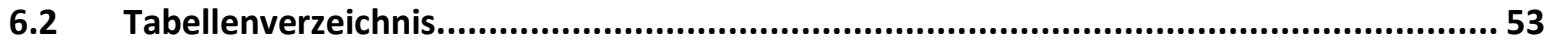

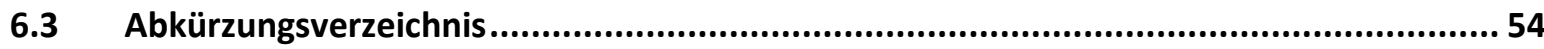

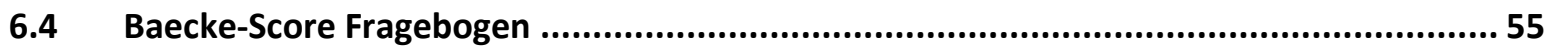

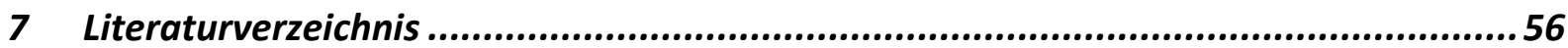




\section{Einleitung}

Die Luxation der Patella ist ein häufiges Krankheitsbild in der Unfallchirurgie und Orthopädie. Die Verrenkung der Patella nach lateral macht ca. 2-3\% aller Knieverletzungen aus und ist gleichzeitig die zweithäufigste Ursache eines Hämarthros im Kniegelenk (ARENDT et al. 2002).

In den letzten Jahren thematisierten zahlreiche klinische und experimentelle Studien die patellofemorale Instabilität, wodurch sich das Verständnis dieser Erkrankung deutlich verbessern konnte. Gleichzeitig hat dieser Erkenntnisgewinn bewirkt, dass das Krankheitsbild der Patellaluxation mehr und mehr ins wissenschaftliche und klinische Interesse gerückt ist. Trotz dieser Fortschritte bleiben verschiedene Fragen bezüglich einiger Prädispositionsfaktoren und der entsprechenden Therapie ungeklärt und werden weiterhin kontrovers diskutiert.

Die Ätiopathogenese des Krankheitsbildes ist vielfältig. An der Stabilität des Patellofemoralgelenks sind knöcherne, ligamentäre und muskuläre Strukturen beteiligt. Um das jeweilige Instabilitätsrisiko zu identifizieren und damit verbunden die entsprechende Therapie zu wählen, ist es entscheidend und von großem klinischem Wert, diese relevanten Stabilitätsfaktoren des Patellofemoralgelenks im Kontext der individuellen Anatomie des einzelnen Patienten zu verstehen (BALCAREK et al. 2010 a, BALCAREK et al. 2010 b).

\subsection{Anatomie des Patellofemoralgelenks}

\subsubsection{Knöcherne Strukturen des Patellofemoralgelenks}

Im Kniegelenk artikulieren drei Knochen miteinander: Femur, Tibia und Patella (Kniescheibe). Dabei wird das Patellofemoralgelenk vom distalen Femur und der Patella gebildet.

Die Patella ist ein dreieckförmiger Knochen. Die Rückseite bildet die Facies articularis patellae, die Vorderseite wird als Facies anterior bezeichnet. Die Gelenkfläche Facies articularis patellae ist mit einer 6-7 mm dicken Knorpelschicht überzogen, der dicksten Gelenkknorpelschicht im Skelettsystem (WHITE und SHERMAN 2009), und wird durch eine vertikale Leiste (Patellafirst) in eine größere mediale und eine kleinere laterale Facette geteilt. Dabei ist der mediale Teil häufig durch eine zweite Leiste noch einmal unterteilt. An der Innenseite der medialen Facette befindet sich eine kleine ovale Knorpelfläche, die so genannte odd facet (GOODFELLOW et al. 1976). Die odd facet tritt bei Flexion $>90^{\circ}$ mit 
dem medialen Femurkondylus in Kontakt und verringert so die druckaufnehmende Fläche im Patellofemoralgelenk.

Die verbreiterten Enden des distalen Femurs bilden den Condylus medialis und lateralis femoris. Dorsal sind die Kondylen durch die Fossa intercondylaris getrennt, während sie ventral durch die Vorderfläche, die Facies patellaris femoris, verbunden werden (MUTSCHLER 1982). Die Facies patellaris femoris wird auch als Trochlea femoris bezeichnet. Sie bildet das Gleitlager für die Patella. Die laterale Femurkondyle prägt dabei die Seitenwand des Patellofemoralgelenks. Sie ist ca. 3,4 mm höher als die mediale Kondyle und spielt deshalb eine wichtige Rolle für die patellofemorale Stabilität. Die normale Trochleatiefe beträgt durchschnittlich 5,2 mm (WALSH 2003).

\subsubsection{Ligamentäre Strukturen des Patellofemoralgelenks}

Das Lig. patellae verbindet die Patella mit der Tibia und ist die bandhafte Fortsetzung der Sehne des M. quadriceps femoris. Es entspringt als kräftiges, flaches Band am unteren Pol der Kniescheibe und inseriert an der Tuberositas tibiae.

Medial und lateral der Patella setzen Sehnenzüge des M. vastus medialis und M. vastus lateralis an und bilden die Retinacula patellae. Sie inserieren beidseits der Tuberositas tibiae und verstärken so die Gelenkkapsel. Die Retinacula gehören zum passiven Stabilisationssystem des Patellofemoralgelenks und teilen sich in eine oberflächliche, eine mittlere und eine tiefe Schicht (WARREN und MARSHALL 1979). Das dünnere oberflächliche Retinaculum verläuft schräg und verbindet die Patella und das Lig. patellae medial mit der Sehne des M. sartorius und der tiefen Beinfaszie. Lateral geht die oberflächliche Schicht in die Fascia lata über (CONLAN et al. 1993, MERICAN et al. 2009). In der mittleren Schicht befindet sich der oberflächliche Teil des Lig. collaterale mediale und das Lig. patellofemorale mediale (MPFL). Am MPFL lassen sich drei Regionen unterscheiden: Femoraler Ursprungsbereich, Mittelteil und Patellaansatzbereich. Seine mittlere Breite beträgt ca. $13 \mathrm{~mm}$ (CONLAN et al. 1993), die durchschnittliche Länge etwa 55-56 mm (TUXOE et al. 2002, MOCHIZUKI et al. 2013). Der Ursprung des MPFL liegt zwischen Tuberculum adductorium und Epikondylus medialis. Von dort verläuft es zum medialen Patellarand und inseriert je nach Studie im Bereich der oberen $2 / 3$ (WARREN und MARSHALL 1979, NOMURA et al. 2005) bzw. in der proximalen 1/2 (AMIS et al. 2003, CONLAN et al. 1993) der medialen Facette. Am Tuberculum adductorium setzt das MPFL gemeinsam mit dem oberflächlichen Teil des Lig. collaterale mediale und der Sehne des M. adductor magnus an (CONLAN et al. 1993). Häufig besteht es aus zwei funktionellen Faserbündeln, wobei einige Fasern zum oberflächlichen Teil des Lig. collaterale mediale ziehen und dort ansetzen (AMIS et al. 2003). Im Verlauf des MPFL kommt es zur Auffächerung der Fasern, sodass sich der patellare Ansatz gewöhnlich breiter darstellt als 
der Ursprungspunkt zwischen dem Tuberculum adductorium und dem Epicondylus medialis (AMIS et al. 2003) und das MPFL sanduhrförmig erscheint. Die distalen Fasern des MPFL sind außerdem mit der tiefen Schicht des medialen Retinaculums verflochten. Dieses ist wiederum mit dem medialen Rand der Patellarsehne verbunden (MOCHIZUKI et al. 2013). Außerdem werden die Patella-nahen Fasern des MPFL teilweise durch distale Fasern des M. vastus medialis obliquus (VMO) überdeckt (AMIS et al. 2003).

Der tiefer gelegene Teil der Retinacula zieht von der Patella zum medialen bzw. lateralen Epikondylus des Femurs und zum vorderen Abschnitt der Menisken (CONLAN et al. 1993, MERICAN et al. 2009). In dieser Schicht befinden sich das Lig. patellotibiale mediale und das Lig. patellomeniscale. Während das Lig. patellomeniscale sekundär stabilisierend auf die Patella wirkt, ist das Lig. patellotibiale mediale funktionell gesehen eher zu vernachlässigen (CONLAN et al. 1993). Ihre Aufgabe ist vor allem propriozeptives Feedback zu geben, um eine Stellungskontrolle der Patella zu erreichen (BODEN et al. 1997).

\subsubsection{Die Entwicklung des Kniegelenks}

Die Morphologie der Trochlea femoris ist schon in utero determiniert. Aus diesem Grund scheinen genetische Ursachen für die Ausprägung einer Patellainstabilität eine entscheidende Rolle zu spielen (GLARD et al. 2005).

Bereits in der 8. Schwangerschaftswoche erreichen die Trochlea bzw. der knorpelige Sulcus die endgültige Form. Die Patella ist so weit entwickelt, dass Bewegungen möglich sind. Die Patella entsteht aus einer kartilaginären Anlage im Bereich der Patellarsehne (GARDNER und O'RAHILLY 1968). Bei der Geburt ist die Kniescheibe knorpelig. Die Verknöcherung beginnt in der frühen Kindheit (CONLAN et al. 1993). Es lassen sich bis zu sechs Ossifikationszentren unterscheiden, die dann zu einem Ossifikationskern zusammenwachsen. Im Normalfall bilden sich zwei patellare Gelenkflächen aus. Diese mediale und laterale Facette sind im physiologischen Fall kongruent zu den Gelenkflächen der korrespondieren Trochlea (NIETOSVAARA 1994).

NIETOSVAARA konnte 1994 in Ultraschalluntersuchungen an Neugeborenen zeigen, dass sowohl die Trochleatiefe wie auch das Patellofemoralgelenk schon bei der Geburt entwickelt sind und dass die Kniegelenksgeometrie bereits denen Erwachsener gleicht. Der knorpelige Sulcuswinkel bei gesunden Kindern ( 0 bis 18 Jahren) betrug in seinen Untersuchungen dabei $134^{\circ}-155^{\circ}$. Gleichzeitig lag der Winkel bei Kindern im Alter von 12 bis 18 Jahren mit bereits vorliegender patellofemoraler Instabilität konsistent erhöht bei $154^{\circ}-195^{\circ}$ (NIETOSVAARA 1994). 


\subsection{Biomechanik des Patellofemoralgelenks}

\subsubsection{Patellofemoraler Bewegungsablauf und die besondere Bedeutung der Patella}

Die Patella ist als das größte Sesambein des menschlichen Skeletts in die Sehne des M. quadriceps femoris eingelagert. Ihre Hauptaufgabe ist die Übertragung von Zugkräften. Dadurch kommt es zu einer Erhöhung des Hebelarms der Extensorenmuskulatur bei Flexions- und Extensionsbewegungen des Kniegelenks und somit $\mathrm{zu}$ einer Kraftverstärkung des M. quadriceps femoris (BACHMANN und PAGENSTERT 2010). Ohne Patella müsste sich die Zugkraft in der Patellarsehne um ca. $40 \%$ erhöhen, um die gleiche Extensionswirkung zu erzielen (KAUFER 1971, BULL und AMIS 2005). Die patellare Führung wird dabei durch die Form der Trochlea, die Form der Patella, die Kraft und Richtung der muskulären Aktivität und durch die Spannung der Retinacula beeinflusst (AMIS et al. 2006).

Das Gleiten der Patella in der Trochlea des Femurs wird auch als Patellatracking bezeichnet (BACHMANN und PAGENSTERT 2010). Bei der Bewegung der Patella gegenüber dem Femur können sechs Freiheitsgrade unterschieden werden: Translation in der mediolateralen, anteroposterioren und kraniokaudalen Richtung sowie Rotation um die Patellalängsachse, um die senkrechte Achse durch das Patellazentrum und um die transversale femorale Achse (BULL et al. 2002).

Die Patella befindet sich dabei zwischen Flexion und Extension in verschiedenen Positionen.

Im vollständig gestreckten Knie rückt die Patella proximal aus dem Patellagleitlager und ist daher in dieser Stellung am mobilsten. Zu Beginn der Flexion befindet sie sich noch nicht vollständig in der Gleitrinne des Femurs, sodass zunächst lediglich der distale Pol der Patella in Kontakt mit dem proximal-lateralen Rand der Trochlea femoris kommt.

In der Flexionsbewegung von $0^{\circ}$ bis $20^{\circ}$ kommt es zu einer Medialisierung der Patella um durchschnittlich $5 \mathrm{~mm}$ (AMIS et al. 2006). Der nach medial gerichteten Bewegung bis $20^{\circ}$ folgt eine Lateralisierung um ca. $6,5 \mathrm{~mm}$ bis in $90^{\circ}$ Flexionsstellung (AMIS et al. 2006). Dabei verhindert die Neigung der lateralen Trochleafacette (Slope) eine Auslenkung der Patella nach lateral (AMIS et al. 2006). Diese Translation wird zudem von einer mediolateralen Kippung (Tilt) der Patella in der Frontalebene begleitet (AMIS et al. 2006).

$\mathrm{Ab} \mathrm{ca} .20^{\circ}$ Flexion wird sie zunehmend durch die knöcherne und knorpelige Geometrie der Trochlea geführt (SENAVONGSE et al. 2003). Bei zunehmender Flexion wandert die Kontaktfläche nach proximal, bis bei $90^{\circ}$ Beugung die gesamte Gelenkfläche der Patella, mit Ausnahme der odd facet, mit der Trochlea femoris artikuliert. Ab $90^{\circ}$ Flexion liegt die Patella quer über der Fossa intercondylaris und befindet sich somit vollständig in der Notch (Bull und AMIS 2005). 


\subsubsection{Die Komponenten der Stabilität des Patellofemoralgelenks}

Im Patellofemoralgelenk werden Stabilität und Mobilität durch ein komplexes Zusammenspiel von aktiven (Muskeln und Sehnen), passiven (Gelenkkapsel und Bänder) und statischen (knöcherne Strukturen) Stabilisatoren bedingt. Beim gesunden Knie interagieren diese Faktoren synergistisch und verhindern so einen Stabilitätsverlust während der Kniebewegung (SENAVONGSE und AMIS 2005, SCHÖTTLE et al. 2009).

a) Aktive Stabilisatoren

Zu den aktiven Stabilisatoren zählen die Muskeln, deren Kräfte an der Patella ansetzen. Von Bedeutung ist hier vor allem der M. quadriceps femoris. Seine vier Köpfe inserieren als gemeinsame Quadrizepssehne am oberen Pol und seitlichen Rand der Patella. Kaudal erfolgt die Fortsetzung als Lig. patellae. Der M. quadriceps besteht aus sechs funktionellen Einheiten. Der M. rectus femoris ist ein zweigelenkiger Muskel. Er überkreuzt das Hüftgelenk. Seine Kraft wirkt dabei parallel zum Femurschaft. Der M. vastus intermedius entwickelt seine Kraft ebenso. Die Mm. vastus lateralis und medialis haben jeweils zwei Funktionseinheiten, einen langen und einen schräg ziehenden Anteil (BULL und AMIS 2005). Biomechanisch betrachtet ist der Quadrizeps der stärkste aktive Stabilisator der Kniescheibe (HUBERTI et al. 1984).

Des Weiteren haben die Muskeln, die am Pes anserinus superficialis ansetzen und zur Muskelgruppe der Beuger gehören, eine aktiv stabilisierende Wirkung. Der Pes anserinus hat dabei auf zwei Ebenen synergistische Auswirkungen. Erstens auf die aktive Spannung im Retinaculum, zweitens auf die Schlussrotation (Screw-home-Mechanismus) bei der endgradigen Streckung im Kniegelenk. Der Unterschenkel und somit die Insertionsstelle des Lig. patellae wird dadurch nach außen gedreht und die lateralisierende Kraft so verstärkt (OLK und HENNIG 2002).

b) Passive Stabilisatoren

Die passiven Stabilisatoren werden durch die Retinacula und insbesondere durch das MPFL geprägt. Das MPFL wirkt der Lateralisierungstendenz der Patella vor allem in Extension und beginnender Flexion $\left(0^{\circ}\right.$ bis $\left.20^{\circ}\right)$ entgegen. Biomechanische Studien haben gezeigt, dass es in diesem Bereich bis zu $60 \%$ der medialen Stabilität bewirkt (CONLAN et al. 1993, DESIO et al. 1998, SENAVONGSE und AMIS 2005) und somit der stärkste passive Stabilisator gegen laterale Instabilität ist (SCHÖTTLE et al. 2009). Bei Knieflexion ab $30^{\circ}$ nimmt die Funktion des MPFL ab, es wirkt jedoch weiterhin stabilisierend auf das Patellofemoralgelenk (SCHÖTTLE et al. 2009, HENSLER und SCHÖTTLE 2010).

c) Statische Stabilisatoren

Dritter wesentlicher Stabilitätsfaktor ist die knöcherne Gelenkgeometrie. Entscheidend für das Gelenk ist dabei die Form der Trochlea (AMIS et al. 2006). Für die Stabilität und Führung der Patella ist die Ausprägung der Trochlearinne verantwortlich (DEJOUR 1994). 
Die aktiven, passiven und statischen Stabilisatoren haben in Abhängigkeit der Kniegelenkstellung unterschiedliche Bedeutung. Während die Patella im Bereich von $0^{\circ}$ bis $30^{\circ}$ eher weichteilig geführt wird, überwiegt die knöcherne Führung bei stärkerer Flexion (FULKERSON 2004).

Im gestreckten Knie wird die Patella vor allem durch das mediale Retinaculum, insbesondere durch das MPFL, und durch die Muskulatur gesichert. Sie wird dabei durch das MPFL in medial-posteriorer Richtung gehalten. Dieses ist aufgrund der Quadrizepsaktivität im gestreckten Knie gespannt. Die Kraft des Quadrizeps zieht die Patella nach proximal und leicht nach lateral (SENAVONGSE und AMIS 2005).

Sobald das Kniegelenk gebeugt wird, beginnt der Lauf der Patella nach medial. Dadurch kommt es gleichzeitig zu einem Spannungsverlust des MPFL, was wiederum eine Minderung seiner stabilisierenden Funktion zur Folge hat (SENAVONGSE und AMIS 2005). Wird das Knie mehr als $30^{\circ}$ gebeugt, rückt die Patella vollständig ins Patellagleitlager und die knöchernen Strukturen stabilisieren die Patella nun nach medial und lateral (OSTERMEIER und BECHER 2010). Zusätzlich wird sie durch die Quadrizepsmuskulatur gesichert, da die Kraftvektoren des M. vastus medialis in dieser Position entgegen der Luxationsrichtung wirken (SCHÖTTLE et al. 2009).

\subsection{Grundlagen der Patellaluxation}

\subsubsection{Definition und Allgemeines}

Die Patellaluxation bezeichnet eine Verrenkung der Kniescheibe aus ihrem femoralen Gleitlager. Dies geschieht meist während der Knieflexion. Die Luxation erfolgt in der Regel nach lateral (STROBL und GRILL 1998, DIEHL und GARRETT 2003, NIKKU et al. 2009). Davon abzugrenzen ist die Subluxation, bei der die Patella seitlich verkippt, das femorale Gleitlager aber nur teilweise verlässt.

\subsubsection{Luxationsformen}

Man unterscheidet nach der Ätiologie des Luxationsereignisses verschiedene Formen der patellofemoralen Instabilität: die akute traumatische Patellaluxation, die akute dispositionelle Patellaluxation, die rezidivierende Luxation, die habituelle Patellaluxation, die chronische Luxation, die kongenitale und die iatrogene Patellaluxation (LEITLINIE Patellaluxation 2014).

Ursache der akuten traumatischen Luxation sind häufig ein direktes adäquates Trauma durch Sturz auf das Knie, Verdrehunfälle beim Sport oder ein seitliches Anpralltrauma 
(DEJOUR et al 1994, NIKKU et al. 2009, BALCAREK et al. 2010 a). Die Patella luxiert nach lateral. Das Luxationsereignis wird meist als sehr schmerzhaft beschrieben (HAWKINS et al. 1986) und ist häufig mit einem Sturz verbunden. Es kommt zu einem plötzlichen Funktionsverlust des Kniegelenks. Die Reposition erfolgt entweder spontan bei reflektorischer Anspannung des Quadrizeps oder sollte sonst schonend unter Analgesie durchgeführt werden. Bei spontaner Reposition wird das eigentliche Luxationsereignis vom Patienten teilweise gar nicht als solches wahrgenommen. Bleibt nach einer ersten traumatisch bedingten Luxation eine Instabilität mit Luxationsneigung bestehen, so spricht man von einer chronisch-rezidivierenden Patellaluxation (LEITLINIE Patellaluxation 2014).

Die akute dispositionelle Patellaluxation hingegen wird durch inadäquate Traumata oder Gelegenheitsursachen bedingt durch prädisponierende Faktoren (siehe 2.4.1) ausgelöst (LEITLINIE Patellaluxation 2014). Bei dieser anlagebedingten Patellainstabilität kommt es schon bei Alltagsbewegungen zu einer Luxation oder Subluxation nach lateral. Es ist keine oder nur eine sehr geringe Wirkung von außen notwendig, um die Stabilität im Patellofemoralgelenk zu stören. Die Reposition erfolgt in den meisten Fällen spontan (HAWKINS et al. 1986, BUSCH 2001). Eng damit verbunden ist die habituelle Patellaluxation. Diese beschreibt willkürlich auslösbare Luxationsereignisse (LEITLINIE Patellaluxation 2014).

Bei der kongenitalen Form ist die Patella bereits bei der Geburt luxiert. Dies tritt meist in Kombination mit einem Genu valgum auf.

Bei der iatrogenen Patellaluxation kommt es als Folge der Therapie einer lateralen Instabilität zu einer Luxation oder Subluxation der Kniescheibe nach medial (LEITLINIE Patellaluxation 2014).

\section{4 Ätiopathogenese der Patellaluxation}

\subsubsection{Prädisponierende Faktoren}

Das Patellofemoralgelenk ist durch die Interaktion der verschiedenen stabilisierenden Faktoren prädisponiert für Instabilitätsleiden. Pathologische Veränderungen der anatomischen Gegebenheiten durch Traumata oder genetisch bedingte Deformitäten können das Gleichgewicht aus statischen, dynamischen und passiven Stabilisatoren stören und so zu einem veränderten Bewegungsverhalten der Patella und damit zu einer patellofemoralen Instabilität führen (SCHÖTTLE et al. 2009).

Nach DEJOUR et al. (1994) können Haupt- und Nebenfaktoren der patellofemoralen Instabilität unterschieden werden: Zu den Hauptfaktoren zählen die Trochleadysplasie, der Patellahochstand (Patella alta), ein erhöhter Abstand der Tuberositas tibiae zur trochleären 
Grube (Tuberositas tibiae-trochlear groove, TT-TG-Abstand) und eine erhöhte nach lateral gerichtete Verkippung der Patella in der Frontalebene (Patella-Tilt). Nebenfaktoren sind eine erhöhte femorale Antetorsion, eine erhöhte tibiale Außenrotation, das Genu valgum und das Genu recurvatum. Zusätzliche Risikofaktoren können eine Hypoplasie des M. vastus medialis, hyperlaxe ligamentäre Strukturen und ein straffer lateraler Bandapparat sein (DEJOUR et al 1994, NIKKU et al. 2009, BALCAREK et al. 2010 a, LEITLINIE Patellaluxation 2014).

In biomechanischen Studien wurden die Auswirkungen der Trochlea-Geometrie, der Retinacula und die Folgen von muskulären Defiziten auf die Patellastabilität untersucht.

Sehr häufige Ursachen der Patellainstabilität sind knöcherne Pathologien. Die Trochleadysplasie konnte durch verschiedene Studien als Hauptrisikofaktor für Patellaluxationen identifiziert werden: bei etwa $96 \%$ der Patienten mit patellofemoraler Instabilität wurde begleitend eine Trochleadysplasie diagnostiziert (DEJOUR et al. 1994, ARENDT et al. 2002, PALMU et al. 2008). Eine abgeflachte Trochlea reduziert die laterale Stabilität bei $30^{\circ}$ Knieflexion um 70 \% (SENAVONGSE und AMIS 2005).

Die Klassifikation der Trochleadysplasie nach Dejour erfolgte 1998 und sieht eine qualitative Einteilung in die Schweregrade Typ A bis D vor. Typ A als leichte Form der Dysplasie beschreibt eine Abflachung der Trochlea mit erhaltenem, aber vermindertem Sulcuswinkel $>145^{\circ}$, Typ B eine flache oder beginnend konvexe Form, Typ C eine konvexe laterale Facette und eine hypoplastische mediale Facette und Typ D zeigt eine vollständige Asymmetrie mit knöchernen Vorsprüngen („cliff pattern“) (DEJOUR et al. 1998).

Zur quantitativen Beurteilung der Trochleadysplasie können der Sulcuswinkel, die Trochleatiefe oder die Trochlea-Facetten-Asymmetrie gemessen werden (NIETOSVAARA 1994, PFIRRMANN et al. 2000). Zur Analyse dieser Veränderungen eignen sich transversale MRT-Schnittbilder oder aber exakt seitliche Röntgenbilder. Parameter im konventionellen Röntgenbild sind das crossing sign, der supratrochleäre Sporn und die Doppelkontur (DEJOUR et al. 1994). Bei einer abgeflachten oder sogar konvex geformten Trochlea fehlt das femorale Gleitlager. Dadurch kommt es in Kombination mit dem dabei gleichbleibenden Quadrizepsvektor zu einem erhöhten patellaren Tilt und Shift (SCHÖTTLE et al. 2009).

Ein weiterer Risikofaktor für ein instabiles Patellofemoralgelenk ist der Patellahochstand, die Patella alta. Bei einer Patella alta gleitet die Kniescheibe erst bei stärker gebeugtem Kniegelenk sicher in der Trochlea. Dadurch nimmt der Bewegungsbereich, in dem die Patella primär durch Weichteilstrukturen gesichert ist, zu. Dies erhöht das Risiko für Instabilitäten (WALSH 2003, OSTERMEIER und BECHER 2010). Um die Patellahöhe zu bestimmen, nutzt man am häufigsten den Index von INSALL und SALVATI: Verhältnis der 
Patellalänge zur Länge des Lig. patellae. Ein Wert von 0,8 bis 1,2 gilt als normal, Werte $>1,2$ als Patellahochstand (INSALL und SALVATI 1971).

Ein zusätzlicher Faktor für die patellofemorale Instabilität kann ein erhöhter Tuberositas-tibiae-Trochlear-groove-Abstand (TT-TG-Abstand) sein. Dieser kann mittels MRT- oder CT- Aufnahmen bestimmt werden und repräsentiert den nach lateral gerichteten Zugvektor des M. quadriceps in der Frontalebene. Die Größe des TT-TG-Abstandes kann isoliert erhöht sein, wird aber auch durch die femorale Antetorsion, die tibiale Außentorsion und durch die Ausprägung der Trochleadysplasie mitbestimmt. (OSTERMEIER und BECHER 2010). In bisherigen Studien konnte kein einheitlicher Wert festgestellt werden, ab dem der TT-TG-Abstand als pathologisch angenommen werden sollte. Die Angaben variieren dabei je nach Autor zwischen 15 und 20 mm (DEJOUR et al. 1994, KOETER et al. 2007). Die Messung des TT-TG-Abstandes hat mittlerweile die klinische Bestimmung des Q-Winkels aufgrund der besseren Reproduzierbarkeit abgelöst und kann als Indikator für einen distalen Korrektureingriff herangezogen werden (BALCAREK und FROSCH 2012).

$\mathrm{Zu}$ den Torsionsfehlern gehören eine vermehrte Antetorsion des Femurs und eine verstärkte tibiale Außentorsion (LEITLINIE Patellaluxation 2014).

Bei bestehender knöcherner Pathologie mit Valgus- und Innentorsionskomponente des Femurs kommt es zu einer relativen Lateralisation der Patella durch die Medialisierung der Trochlea. Die Patella kann bei zunehmender Flexion nicht mehr in das Patellagleitlager eintauchen - es kommt zur dauerhaften Subluxation. Die Patella „reitet" auf der lateralen Femurkondyle (BALCAREK und FROSCH 2012).

Neben den genannten, die Gelenkgeometrie betreffenden Prädispositionen sind die passiven und dynamischen Stabilisatoren für die patellofemorale Stabilität mitentscheidend.

In verschiedenen Studien wurde dabei insbesondere die Funktion des MPFL untersucht. Diese Untersuchungen zeigten, dass eine Schwächung bzw. Verletzung des MPFL gravierende Auswirkungen auf die patellofemorale Stabilität haben können (CONLAN et al. 1993, DESIO et al. 1998). Den stärksten Effekt hat eine MPFL-Ruptur in strecknaher Stellung des Kniegelenks mit einer Reduktion der lateralen Stabilität um $49 \%$ (SENAVONGSE und AMIS 2005). Folglich wird ein funktionell eingeschränktes MPFL als eine der Hauptursachen für rezidivierende Luxationen angesehen (NOMURA 1999).

Häufig ist das MPFL bereits bei Kindern chronisch insuffizient. Als Grund wird eine früh einsetzende Fehlstellung der Patella postuliert. Häufigste Ursache der Patellalateralisation ist die Trochleadysplasie. Bei einer abgeflachten oder sogar konvex geformten Trochlea fehlt das femorale Gleitlager. Dadurch kommt es in Kombination mit dem dabei gleichbleibenden Quadrizepsvektor zu einem erhöhten patellaren Tilt und Shift 
(SCHÖTTLE et al. 2009). Gleichzeitig können Valgus- und Innentorsionsfehlstellungen die Lateralisation der Patella begünstigen. Bei diesen Achsfehlstellungen liegt der Sulcus der Trochlea femoris medialisiert, während die Patella dadurch relativ gesehen lateralisiert ist. Als Konsequenz kann sich das MPFL nicht funktionsentsprechend entwickeln (SCHÖTTLE et al. 2009). Es kommt zur MPFL-Insuffizienz bzw. zur MPFL-Hyperlaxizität.

\subsection{Der M. vastus medialis obliquus und seine Funktion im Patello- femoralgelenk}

\subsubsection{Anatomie und Biomechanik des VMO und seine Bedeutung für die Stabilität des PFG}

Der $\mathrm{M}$. vastus medialis obliquus (VMO) ist der distale, schrägverlaufende Anteil des $\mathrm{M}$. vastus medialis (s. Abb. 1). Seine Muskelfasern ziehen in einem Winkel von ca. $50^{\circ}$ und setzen horizontal am medialen Patellarand an. Die Insertionsstelle befindet sich dabei in der oberen Hälfte der Patella (LIN et al. 2008). Die Angaben zu den VMO-FaserverlaufswinkeIn variieren je nach Autor. In der Studie von SAKAI et al. (2000) betrug der Winkel zwischen VMO und $M$. rectus femoris im Mittel 42,5 $\left( \pm 1,5^{\circ}\right)$. HINTON und SHARMA (2003) gaben für die Ausrichtung der Muskelfasern Werte von etwa 50-60 an. Nach FAHRAMAND et al. (1998) zogen die Fasern des VMO in einem Winkel von ca. $47^{\circ}\left( \pm 5^{\circ}\right)$ zur Femurlängsachse, und RAIMONDO et al. beschrieben 1998 in ihren Untersuchungen einen Muskelfaserwinkel von etwa $52^{\circ}$ sowie im Durchschnitt eine Querschnittsfläche von 3,1 $( \pm 1,3) \mathrm{cm}^{2}$.

Patella-nah besteht häufig eine Verbindung zwischen dem VMO und dem MPFL. Die distalen Fasern des VMO überdecken die Fasern des MPFL, und es entsteht ein gemeinsamer Ansatzpunkt von VMO und MPFL am medialen Patellarand (AMIS et al. 2003). In einer aktuellen Studie von MOCHIZUKI et al. (2013) ergaben sich diesbezüglich abweichende Ergebnisse. Laut ihren Untersuchungen seien die proximalen Fasern des MPFL hauptsächlich am medialen Rand der Sehne des M. vastus intermedius befestigt.

Eine feste Anheftungsstelle zwischen MPFL und M. vastus medialis zeigte sich hingegen in ihrer Studie nicht; hier ergab sich nur eine lose Verbindung zwischen MPFL und dem distalen medialen Bereich des M. vastus medialis. Die distalen MPFL-Fasern seien vor allem mit der tiefen Schicht des medialen Retinaculums verflochten. Das MPFL sei demnach direkt mit dem M. vastus intermedius und der Patella sowie indirekt über die distalen MPFL-Fasern mit der Patellarsehne verbunden. Die Breite des MPFL-Ansatzes am medialen Rand des M. vastus intermedius belief sich in der Studie von MOCHIZUKI et al. (2013) auf ca. $24 \mathrm{~mm}$. 
Des Weiteren wurde von MOCHIZUKI et al. (2013) ein schräger Verlauf des Hauptteils der Muskelfasern des M. vastus medialis beschrieben. Der Großteil der Fasern inserierte an der Sehne des M. vastus intermedius, während nur wenige distal gelegene Fasern direkt am superior-medialen Rand der Patella ansetzten

Topographisch betrachtet schließen MPFL, VMO und die Sehne des M. adductor magnus einen dreieckigen Bereich ein (s. Abb. 1). Dieser befindet sich distal des VMOs, proximal des MPFLs und anterior der Sehne des M. adductor magnus (AMIS et al. 2003).

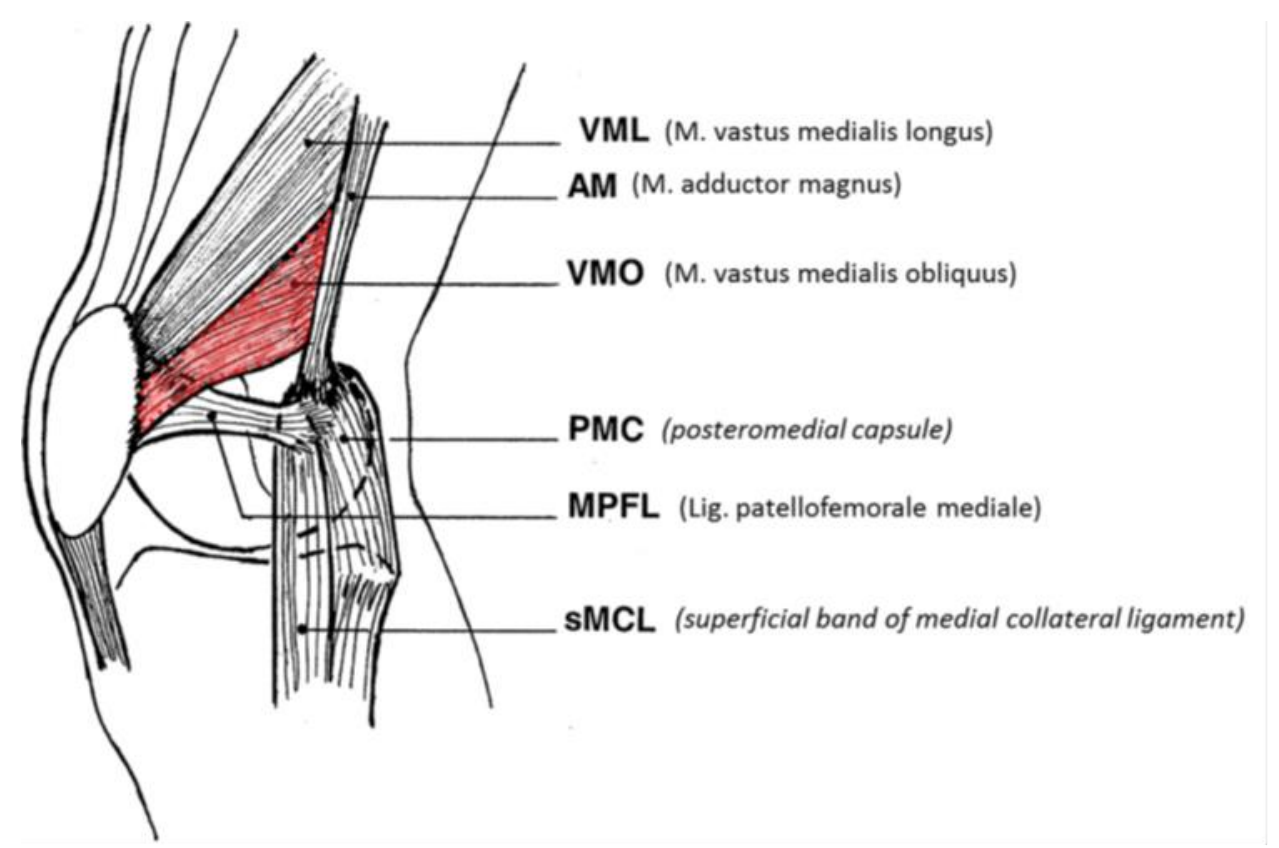

Abbildung 1: Topographische Lage des MPFL zu seinen benachbarten Strukturen (modifiziert nach Amis et al. 2003, S. 216).

Elektromyographische Studien, die die unterschiedliche Aktivierung von VMO und M. vastus lateralis untersuchten, haben gezeigt, dass der VMO im Bereich von 0-30 Flexion stärker aktiviert wird. Im Gegensatz dazu kommt dem lateralen Anteil des M. quadriceps zwischen $30^{\circ}$ und $90^{\circ}$ Knieflexion stärkere Bedeutung zu (MARIANI und CARUSO 1979). Insgesamt stehen M. vastus medialis und lateralis jedoch in synergistischer Beziehung und im gesunden Knie im Gleichgewicht zueinander. Ihre distalen schräg verlaufenden Anteile (VMO und VLO) üben von medial und lateral eine transversal gerichtete Kraft auf die Patella aus (BENNET et al. 1993). Sie sind somit neben Faktoren wie z.B. einer möglichen Achsfehlstellung, einer veränderten knöchernen Gelenkgeometrie oder geschwächten Weichteilstrukturen mitentscheidend für die Positionierung der Patella in der Trochlea (FAHRAMAND et al. 1998, LIN et al. 2008). Daher wird auch vermutet, dass diese Komponenten trotz ihres eigentlich geringen Anteils relativ zum Gesamtquerschnitt des M. quadriceps den größten Anteil am Gleichgewicht der medio-lateralen Patellabewegung haben (FAHRAMAND et al. 1998). 
Es darf angenommen werden, dass der VMO als Teil des M. quadriceps femoris aufgrund seiner schräg verlaufenden Fasern eine entscheidende Rolle als medial dynamisch wirkender Stabilisator für das patellofemorale Tracking und die patellofemorale Stabilität spielt (LIEB und PERRY 1968, GOH et al. 1995, HINTON und SHARMA 2003). Durch seinen Muskelfaserverlauf ist seine Kraft nach medial, posterior und proximal gerichtet (FAHRAMAND et al. 1998, BULL und AMIS 2005). LIEB und PERRY beschrieben 1968 in ihren Untersuchungen vor allem die große nach medial gerichtete Kraftkomponente, während BOSE et al. (1980) den nach posterior gerichteten Kraftvektor hervorhoben. Durch diese kombinierte Wirkung wird die Patella bei Kontraktion des VMO stabilisiert (LIEB und PERRY 1968, BOSE et al. 1980).

Eine VMO-Atrophie wird daher auch als ein entscheidender Faktor für die Entwicklung von Patella(sub)luxationen gesehen (SAKAl et al. 2000). Jedoch variieren die Aussagen in der Literatur bezüglich des VMO-Anteils an der stabilisierenden Wirkung während der Kniebewegung. Einige Autoren schreiben dem VMO vor allem im Bereich der terminalen Knieextension eine stabilisierende Funktion zu (LIEB und PERRY 1968, GOH et al. 1995, OLK und HENNIG 2002). Andere hingegen fanden nur geringe Effekte einer VMO-Relaxation im gestreckten Knie. SENAVONGSE et al. konnten in ihren Untersuchungen zeigen, dass eine Relaxation des $\mathrm{VMO}$ bei $20-30^{\circ}$ Flexion eine mittlere Reduktion der lateralen patellofemoralen Stabilität von etwa $30 \%$ bewirke (SENAVONGSE et al. 2003); der Effekt sei mit einer Reduktion der Stabilität von etwa 14 \% im gestreckten Knie am geringsten gewesen (SENAVONGSE und AMIS 2005). FARAHMAND et al. beschrieben 2004 in ihrer Studie sogar, dass der VMO erst ab 60 Flexion, wenn er senkrecht zur Luxationsrichtung der Patella verlaufe, das Patellofemoralgelenk stabilisiere. Außerdem sei er nur dann funktionsfähig, wenn er mit dem proximalen Anteil des MPFL anatomisch verbunden ist (FARAHMAND et al. 2004). Die Bedeutung eines insuffizienten

VMO als Prädispositionsfaktor für die Patellaluxation bedarf daher weiterer Untersuchungen (FARAHMAND et al. 1998).

\subsubsection{Der VMO im Kontext des Krankheitsbildes der Patellaluxation und des patellofemoralen Schmerzsyndroms - aktuelle Studienlage}

Während wie unter 1.5.1 erläutert der Stellenwert eines insuffizienten VMO für die Entwicklung der patellofemoralen Instabilität in der Literatur kontrovers diskutiert wird, ist die Bedeutung des VMO für das patellofemorale Schmerzsyndrom (PFPS) bereits relativ gut untersucht.

Das PFPS ist ein verbreitetes Krankheitsbild, sowohl in der Normalbevölkerung wie auch unter Sportlern. Die Inzidenz in der Normalbevölkerung beträgt ca. $25 \%$, während sie im Sportbereich sogar höher liegt (DEVEREAUX und LACHMANN 1984). Ein genauer 
Auslöser bzw. die Ursache des PFPS konnte bis heute nicht definiert werden angenommen wird eine multifaktoriell bedingte Pathophysiologie. Als entscheidender Faktor gilt ein patellofemorales Maltracking. Zahlreiche Studien konnten darüber hinaus einen Zusammenhang zwischen PFPS, patellofemoralem Maltracking und VMO-Atrophie belegen (LIEB und PERRY 1968, BOSE et al. 1980, LIN et al. 2008). Durch ein Ungleichgewicht in der Aktivität von $\mathrm{M}$. vastus medialis obliquus relativ zum M. vastus lateralis kommt es zu einem gestörten Patellagleiten (SAKAl et al. 2000). Diskutiert wird, dass es dabei infolgedessen zu einer Insuffizienz und sekundären Atrophie mit Hypoplasie des VMO kommt bzw. dass es erst durch den geschwächten Muskel zum Maltracking kommt (FOX 1975, PATTYN et al. 2011). Daher wird das PFPS auch im Zusammenhang mit einer abnehmenden VMO-Muskelmasse gesehen. Diesbezüglich wurde in verschiedenen Studien die VMO-Atrophie bei PFPS-Patienten im Vergleich zu gesunden Kontrollpatienten untersucht. Eine 1996 von POWERS et al. durchgeführte Untersuchung ergab, dass der VMO bei patellofemoralen Schmerzen stärker zur Schwächung neige, als die übrigen Komponenten des M. quadriceps (POWERS et al. 1996). JAN et al. (2009) konnten in ihrer Studie mittels Sonographie ein signifikant kleineres VMO-Volumen bei PFPS-Patienten verglichen mit gesunden Kontrollpatienten feststellen. Die MRT-gestützte Untersuchung von KAYA et al. (2011) zeigte ein vermindertes Gesamtvolumen und eine reduzierte Querschnittsfläche des M. quadriceps in der PFPS-Gruppe. Die ebenfalls mittels MRT durchgeführte Studie von PATTYN et al. (2011) ergab eine signifikant geringere VMO-Querschnittsfläche in der Gruppe der PFPS-Patienten. Im Vergleich zur gesunden Kontrollgruppe war die Querschnittsfläche hier $2 \mathrm{~cm}^{2}$ kleiner. Nicht geklärt werden konnte, ob die VMO-Atrophie der Ausbildung eines PFPS vorausgeht, oder ob die VMO-Atrophie bedingt durch das PFPS entsteht.

Leroux et al. (1995) vermuten diesbezüglich, dass die spezifische Atrophie des VMO eher durch den funktionellen Unterschied, beschrieben durch LIEB und PERRY (1968), zwischen den einzelnen Quadrizepskomponenten zustande komme. Der VMO setze direkt an der Patella an und bewirke so eine mediale Patellabewegung ohne Einfluss auf die Kniestreckung zu haben. Der M. vastus lateralis und die proximalen Fasern des M. vastus medialis hingegen inserierten in der Quadrizepssehne und würden so zusammen mit dem M. rectus femoris aktiv auf die Knieextension wirken.

Des Weiteren wurde durch mehrere Autoren beschrieben, dass der VMO bei Patienten mit PFPS nicht, wie normalerweise, im Bereich der oberen Patellahälfte inseriere, sondern sich proximaler befinde und der Faserverlauf verändert sei (BOSE et al. 1980, HUBBARD et al. 1997, LIN et al. 2010). Angenommen wird außerdem, dass der VMO durch Kontraktion aufgrund seiner anatomischen Lage den lateralen Shift und Tilt der Patella begrenze $(\mathrm{GOH}$ et al. 1995, SAKAl et al. 2000). LIN et al. stellten diesbezüglich 2008 in ihren 
Untersuchungen ebenfalls einen Zusammenhang zwischen VMO und Patella-Malalignement bei PFPS-Patienten fest. Die Querschnittsfläche des VMO korrelierte signifikant mit den Parametern für Patella-Malalignement. Diese Korrelation war in ihrer Studie bei Patienten mit verstärktem Patella-Tilt und lateralem Patella-Shift am größten (LIN et al. 2008).

Die Assoziation von patellofemoralem Maltracking und VMO-Schwäche wird durch die Entwicklung des Quadrizeps unterstützt. Der M. vastus medialis ist der letzte Teil des M. quadriceps, der sich entwickelt und der Erste, der bei „Nichtnutzen“ atrophiert bzw. der letzte, der rehabilitiert (FOX 1975). Daher neigt der VMO bei Schmerzen im Patellofemoralgelenk zur Atrophie und regeneriert bei Rehabilitation zuletzt.

Bezüglich des Krankheitsbildes der Patellaluxation scheint die anatomische Verbindung von MPFL und VMO ein entscheidender Faktor zu sein. Lagebedingt sind beide Strukturen häufig zusammen verletzt. Studien zeigten, dass der VMO bei Patellaluxationen dabei häufig fortschreitend nach proximal in Richtung der Sehne des M. adductor magnus einreißt (AHMAD et al. 2000). Vor allem für akute Erstluxationen postulieren verschiedene Autoren, dass die Verletzung des MPFL häufig in einer Ablösung der distalen VMO-Fasern bzw. des VMO-Ansatzes an der Patella resultiere. Dies habe dann eine Verlagerung des VMO nach superior und lateral und damit eine Veränderung der Zugrichtung bzw. des Kraftvektors der VMO-Fasern zur Folge (SALLAY et al. 1996, DESIO et al. 1998, AHMAD et al. 2000).

\subsubsection{Prinzipien der konservativen Patellaerstluxationstherapie und die Rolle des VMO}

Die Therapie der unkomplizierten Erstluxation (ohne osteochondrale Flake-Fraktur) ist in der Literatur weiterhin kontroverser Diskussionspunkt (BALCAREK und FROSCH 2012).

Nach aktueller Lehrmeinung wird derzeit zunächst eine konservative Therapie empfohlen. Dies ist durch Studienergebnisse der letzten Jahre begründet, die keinen Vorteil einer frühen operativen Versorgung gegenüber einer konservativen Therapie fanden (NIKKU et al. 1997, NIKKU et al. 2005, PALMU et al. 2008). Die Indikation zur konservativen Therapie stellt sich hierbei unter Berücksichtigung der individuellen Wahrscheinlichkeit für eine Rezidivluxation nach Analyse der Prädispositionsfaktoren und des Verletzungsmusters.

Die konservative Therapie umfasst symptomorientierte Maßnahmen (Kühlung, Analgesie), sowie die stufenweise limitierte Flexion für einen Zeitraum von insgesamt 6 Wochen mittels 4-Punkte-Orthese. Außerdem solle ein gezieltes Muskelaufbau- und Koordinationstraining erfolgen (SCHÖTTLE et al. 2009)

Aufgrund der vermuteten besonderen Funktion des VMO für die patellofemorale Stabilität wird durch verschiedene Autoren der Fokus bei der Therapie der patellofemoralen 
Instabilität auf die spezielle Kräftigung des VMO gelegt. Die Therapie der M. vastus medialis Insuffizienz soll dabei isometrische, isotonische sowie isokinetische Muskelkräftigungsübungen umfassen. DOUCETTE und GOBLE (1992) untersuchten den klinischen und radiographischen Effekt dieser gezielten VMO-Kräftigung und schlussfolgerten, dass entsprechende Übungen das laterale Patellatracking verbessern könnten.

ATKIN et al. (2000) empfehlen zunächst Übungen in geschlossener Kette und heben dabei ebenfalls die Kräftigung des VMO hervor.

Außerdem wird zum Teil das Tapen der Patella empfohlen. GILLEARD et al. (1998) untersuchten den Effekt des Patella-Tapings bzgl. der VMO-Aktivität bei PFPS. Dies solle bewirken, dass der VMO früher als der VL aktiviert werde. Dies habe dann günstige Effekte auf die patellofemorale Mechanik. Das Tapen unterstütze dabei das frühe Einrücken der Patella in die Trochleagrube (GILLEARD et al. 1998).

\subsection{Zielsetzung dieser Arbeit}

Charakteristisch für das Entstehen einer Patellaluxation ist ein Ungleichgewicht zwischen den aktiven, passiven und statischen Stabilisatoren des Patellofemoralgelenks (FITHIAN et al. 2004, SENAVONGSE und AMIS 2005, BALCAREK et al. 2010 b). In dieser Arbeit sollen die morphologischen Eigenschaften des VMO im Kontext der patellofemoralen Instabilität und seine Bedeutung als prädisponierender Faktor für eine Patellaluxation weiter untersucht werden.

Verschiedene Studien schlussfolgerten, dass der VMO-Muskel einen besonderen Wert für die patellofemorale Stabilität hat (LIEB und PERRY 1968, FARAHMAND et al. 1998, HINTON und SHARMA 2003, RHEE et al. 2012). Vor allem in Bezug auf Patella-Shift, - Tilt und die aufzubringende Kraft, um die Patella zu lateralisieren, habe der VMO eine entscheidende antagonistische Funktion (SAKAI et al. 2000, SENAVONGSE und AMIS 2005).

Während die Auswirkungen einer VMO-Atrophie, eines Ungleichgewichts zwischen VMO und VL sowie eines veränderten neuromuskulären Ansprechens der verschiedenen Quadrizepskomponenten im Hinblick auf das Patellofemorale Schmerzsyndrom bereits relativ gut verstanden sind, fehlen vergleichbare Daten für die patellofemorale Instabilität. Insbesondere der stabilisierende Effekt des VMO bei Existenz einer typischen anatomisch-instabilen Patella mit Trochleadysplasie, Patella alta und vergrößertem TT-TG-Abstand wurde bisher nicht beschrieben. 
Besonders unter Berücksichtigung der konservativen Therapiekonzepte, die nach primärer Luxation besonderen Fokus auf die Rehabilitation und Kräftigung des Quadrizepsmuskels legen (HING et al. 2011, VAVKEN et al. 2013), bedarf es einer genaueren Analyse der Bedeutung des VMO für die patellofemorale Instabilität, nicht nur vor dem Hintergrund der anatomisch prädisponierenden Eigenschaft, sondern auch im Hinblick auf die Beurteilung dieser Therapieansätze.

Der Muskelquerschnitt korreliert mit der möglichen Kraft, die ein Muskel aufzubringen vermag und kann reliabel mittels MRT bestimmt werden (MITSIOPOULOS et al. 1998, BERRY et al. 2008, PATTYN et al. 2011). Weitere morphologische Faktoren, die den stabilisierenden Effekt des schräg verlaufenden VMO auf die Patella bestimmen, sind der Muskelfaserwinkel und die kraniokaudale Ausdehnung des Muskels in Relation zur Patella.

Es ergibt sich daher folgende Fragestellung für diese Arbeit:

Unterscheiden sich die morphologischen Parameter des VMO (Querschnittsfläche, Muskelfaserwinkel und kraniokaudale Ausdehnung) bei Patienten mit einer patellofemoralen Instabilität von asymptomatischen Kontrollpatienten?

Geht eine mögliche VMO-Schwäche einer Erstluxation voraus oder entwickelt diese sich sekundär als Folge einer schmerzbedingten physischen Inaktivität nach rezidivierenden Luxationen?

Unterscheiden sich die morphologischen Parameter des VMO unter Berücksichtigung der prädisponierenden Pathoanatomie der Patellaluxation (Trochleadysplasie, Patella alta, TT-TG-Abstand)? 


\section{Material und Methoden}

\subsection{Patientenkollektiv}

Im Rahmen dieser Studie wurden Patienten untersucht, die sich in ambulanter oder stationärer Behandlung in der Universitätsmedizin Göttingen (UMG) befanden. Die Untersuchungen fanden im Zeitraum von September 2010 bis Mai 2013 im Rahmen der Kniesprechstunde der UMG statt. Es wurden insgesamt 82 Patienten, die die Einschlusskriterien erfüllten, eingeschlossen.

Die durchgeführte Studie besteht aus drei Patientengruppen:

Gruppe $\boldsymbol{A E}$ schließt 30 Patienten mit einer stattgehabten akuten Patellaerstluxation ein.

Gruppe $C L$ besteht aus 30 Patienten mit chronischer patellofemoraler Instabilität und rezidivierenden Luxationsereignissen in der Anamnese.

Gruppe $K$ bildet die Kontrollgruppe. Sie setzt sich aus 22 Patienten ohne Luxationsereignis oder Erkrankungen des Patellofemoralgelenks zusammen, die kurz zuvor ein anderes Kniegelenkstrauma erlitten haben.

Da diese Studie auf der Auswertung von MRT-Bildern basiert, war für die Auswahl der Patienten das Vorhandensein einer MRT-Untersuchung des betroffenen Kniegelenks entscheidend. Die MRT-Bildgebung wurde sowohl in der Gruppe AE (Median 6 Tage zwischen Verletzung und MRT) wie auch der Kontrollgruppe (Median 7 Tage zwischen Verletzung und MRT) innerhalb von 10 Tagen nach Trauma durchgeführt.

Gegenstand dieser Arbeit ist die Untersuchung der muskulären Situation der Patienten. Um die Auswirkungen einer Muskelatrophie als Folge der physischen Inaktivität nach der Verletzung so gering wie möglich zu halten, wurde diese kurze Zeitspanne zwischen MRT und Trauma festgelegt. Die MRT-Bildgebung der Gruppe CL wurde innerhalb eines beschwerdefreien Intervalls zeitlich unbegrenzt nach Luxation durchgeführt.

Ausschlusskriterien stellen Alter über 45 Jahre, jegliche Vorerkrankung des Patellofemoralgelenks (außer vorherige Luxationsereignisse in der Gruppe CL), Frakturen des distalen Femurs oder Tibiakopfes, multiligamentäre Kniegelenks-Instabilität, sonstige Erkrankungen des Patellofemoralgelenks und starkes Übergewicht (BMI >35) dar. Weiterhin von der Studie ausgenommen sind Patienten mit bestimmten Vor-Operationen wie z.B. Trochleaplastiken oder femorale Umstellungsosteotomien. Außerdem ausgeschlossen sind Patienten mit Patellaerstluxation, bei denen die Zeitspanne zwischen Verletzung und MRT-Untersuchung mehr als 10 Tage betrug.

Ebenfalls ausgeschlossen sind Patienten mit einer traumatischen Patellaluxation, die sich nach einem adäquaten Trauma von medial oder nach einem direktem Sturz auf die Patella bzw. durch starke Gewalteinwirkung von außen ereignet haben. 


\subsection{Anamnestische Daten}

Erfragt werden Verletzungsursache, Unfalltag und die Umstände des Luxationsereignisses. Des Weiteren wird bei den Patienten erfasst, ob es sich um eine Erst- oder um eine Rezidivluxation handelt. Bei chronischer Instabilität werden die Anzahl der stattgehabten Patellaluxationen, eventuelle Subluxationsereignisse und Instabilitätsepisoden der Gegenseite anamnestisch erhoben. Zusätzlich wird der genaue Verletzungsmechanismus dieser und insbesondere der Mechanismus der Erstluxation erfragt.

Außerdem werden sonstige Verletzungen sowohl des betroffenen wie auch des Kniegelenks der Gegenseite sowie weitere relevante medizinische Vorerkrankungen aufgenommen.

Die Diagnose der lateralen Patellaluxation basiert auf der Anamnese, einer eingehenden klinischen Untersuchung sowie den definierten MRT-Kriterien für laterale Patellaluxationen (BALCAREK et al. 2010).

Die Unfallursache kann in vier Kategorien eingeteilt werden. Nach der „Innsbrucker Knie-Sportfähigkeitsklassifikation“ (Fink et al. 1993) werden High-Risk-Pivoting-, Low-Risk-Pivoting- und No-Risk-Pivoting-Sportarten unterschieden. Zu den High-risk-Pivoting Sportarten zählen u.a. Fußball und Basketball. Sportarten wie z.B. Klettern, Joggen oder Tanzen hingegen gehören in die Gruppe der Low-risk-Pivoting Aktivitäten. Zu den No-risk-Pivoting Sportarten zählen z.B. Schwimmen und Radfahren. Die vierte Kategorie wird durch das direkte Anpralltrauma gebildet. Aus dieser Einteilung ergibt sich die Differenzierung in traumatische und atraumatische Patellaluxationen.

In die Anamnese gehört außerdem die Aufnahme weiterer Prädispositionsfaktoren der Patienten, wie z.B. Achsfehlstellungen (Genu valgum oder recurvatum), Patella- oder Trochleaanomalien (Patella alta, Trochleadysplasien, erhöhter TT-TG-Abstand). Auch eine eventuelle familiäre Vorbelastung wird erfasst.

Um die untersuchten morphologischen Parameter des VMO (Querschnittsfläche, Muskelfaserwinkel und kraniokaudale Ausdehnung) unter den Gruppen vergleichbar zu machen, wurden notwendige demographische Daten der Patienten erhoben. Dazu zählen die Berücksichtigung des Alters, des Body-Mass-Index (BMI) (ermittelt über das Körpergewicht und die Körpergröße) sowie der körperlichen Aktivität der Studienteilnehmer nach BAECKE et al. (1982) (BAECKE-Score). Zur Erhebung der individuellen physischen Aktivität mittels des BAECKE-Scores (siehe Anhang 6.4) wurden zusätzlich Daten wie Beruf, Aktivitätslevel im Alltag und sportliche Aktivität erfragt. Die Gruppen wurden untereinander in Bezug auf die Parameter Geschlecht, Alter und Body-Mass-Index (BMI) sowie ihrer physischen Aktivität (BAECKE-Score) angeglichen. 


\subsection{MRT-Bildgebung}

\subsubsection{MRT-Technik}

Die MRT-Untersuchungen wurden mit einem 1.5-T oder 3.0-T-Magnetresonanztomographen (Magnetom TrioTim Syngo MR B15 und Magnetom Symphony Syngo MR A30, Siemens, Erlangen, Deutschland) durchgeführt.

Das zu untersuchende Kniegelenk der Patienten befand sich während der MRT-Untersuchung in Extension mit entspannter Muskulatur.

Die folgenden Sequenzen werden routinemäßig durchgeführt und wurden für diese Studie genutzt.

Magnetom TrioTim Syngo MR B15: eine transversale fett-gesättigte protonen-dichte-gewichtete Fast-Spin-Echo-Sequenz (Repetitionszeit/Echozeit, 4000/53 Millisekunden; Flipangle, 150; Auflösung / FOW, 200x200 mm; Schichtdicke, $4.0 \mathrm{~mm}$ ) und eine sagittale T1-gewichtete Spin-Echo-Sequenz (Repetitionszeit/Echozeit, 983/17 Millisekunden; Flipangle, 150; Auflösung /FOW, 200x200 mm; Schichtdicke, 3.5 mm).

Magnetom Symphony Syngo MR A30: eine transversale fett-gesättigte protonen-dichte-gewichtete Fast-Spin-Echo-Sequenz (Repetitionszeit/Echozeit, 4500/33 Millisekunden; Flipangle, 150; Auflösung / FOW, 160×160 mm; Schichtdicke, $4.0 \mathrm{~mm}$ ) und eine sagittale T1-gewichtete Spin-Echo-Sequenz (Repetitionszeit/Echozeit, 490/14 Millisekunden; Flipangle, 90; Auflösung / FOW, 220x220 mm; Schichtdicke, 3.0 mm).

\subsubsection{Centricity PACS}

Für die Messungen in dieser Studie wurde das Centricity picture archiving and communication system (PACS) Programm der Firma GE Healthcare, St. Giles, United Kingdom verwendet.

Dieses ermöglicht, auf zwei Bildschirmen die Verbindung zwischen den verschiedenen MRT-Serien mittels Querverweis herzustellen. Des Weiteren lassen sich über verschiedene Funktionen sowohl die unterschiedlichen Winkel wie auch die Querschnittsflächen bestimmen.

\subsection{Erhebung der VMO-Parameter}

\subsubsection{VMO-Querschnittsfläche}

Um die Querschnittsfläche des M. vastus medialis obliquus (VMO) zu bestimmen, wurden die sagittale und transversale Serie der MRT-Untersuchungen verwendet. 
Zunächst wird in der sagittalen Serie die Ebene aufgesucht, in der die Patella vollständig und in ihrer längsten Ausbreitung zu sehen ist (Abb. 2, Bild A). Der obere Patellapol dient als Standardreferenzpunkt $(R)$. Von diesem gelangt man über den Querverweis zum entsprechenden Bild in der transversalen Serie (Abb. 2, Bild C). Im transversalen Schnittbild wird der VMO aufgesucht und mittels der Flächenmessfunktion die VMO-Querschnittsfläche bestimmt. Dazu wird der Umriss des Muskels mit dem Flächenmesser umfahren und durch Punkte markiert. Nach vollständigem Markieren der Außenränder ermittelt das Centricity PACS-Programm automatisch die durchschnittliche Querschnittsfläche des VMO (rote Linie in Bild C, Abb. 2). Diese wird in $\mathrm{mm}^{2}$ angegeben. Zusätzlich wird die VMO-Querschnittsfläche der direkt ober- bzw. unterhalb liegenden Schichten $\left(R_{1}\right.$ und $R_{2}$ ) in Bezug zur Referenzschicht $(R)$ ermittelt (weiße Linien in Bild $B$ und $D$, Abb. 2) (MRT Schichtdicke 3,5 mm). Die drei Werte der VMO-Querschnittsfläche werden summiert und sollen so die dreidimensionale Struktur des Muskels widerspiegeln. 

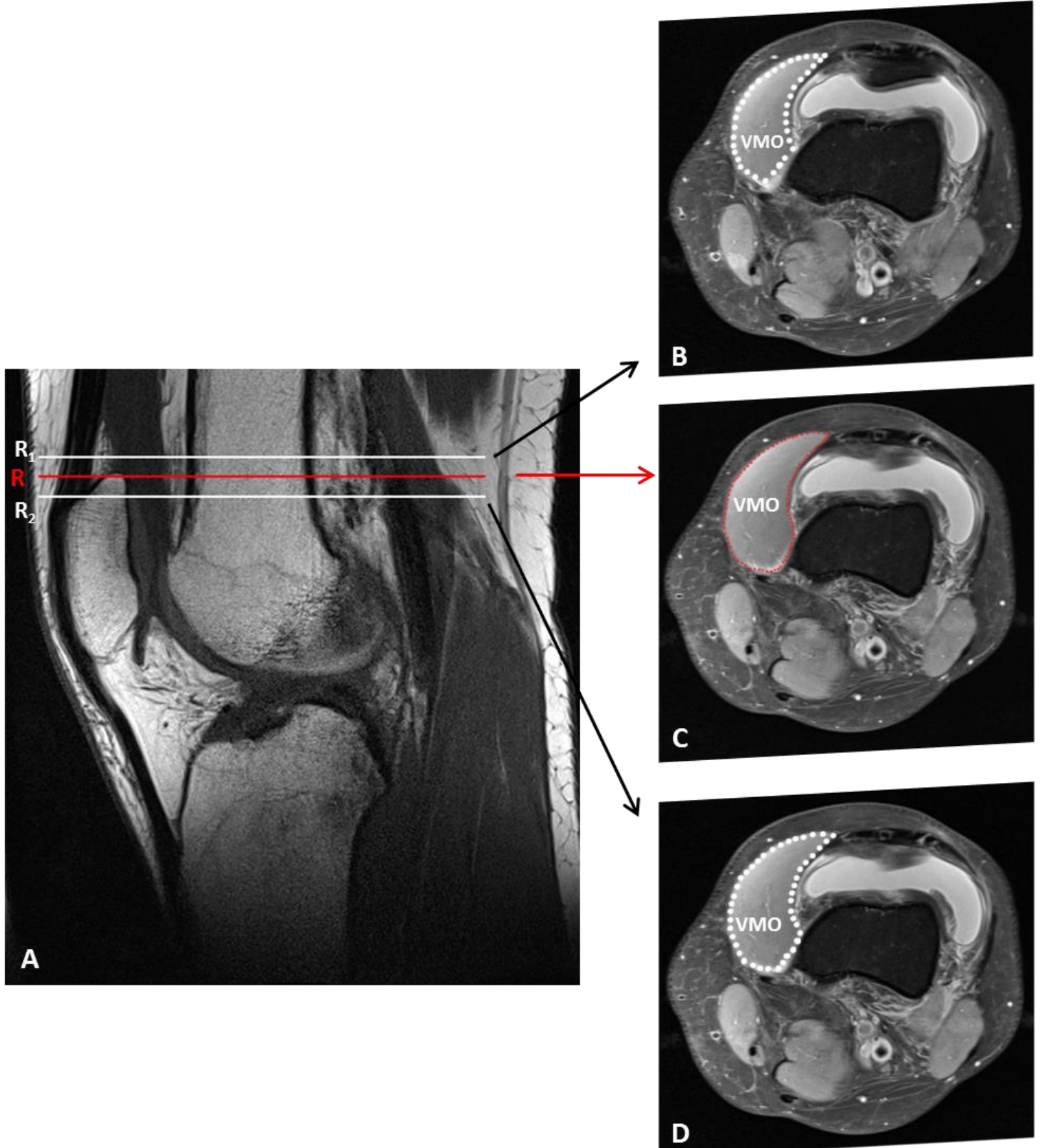

Abbildung 2: Messung der VMO-Querschnittsfläche

$\boldsymbol{A}$ zeigt ein Bild der sagittalen Serie. Über den Querverweis (Referenzlinie R) gelangt man zum entsprechenden Bild $(\boldsymbol{C})$ der transversalen Serie. In $\boldsymbol{C}$ erfolgt mit Hilfe der Flächenmessfunktion über das Markieren des VMOUmrisses die Berechnung der VMO-Querschnittsfläche. Zusätzlich erfolgt die Berechnung der VMO-Querschnittsfläche der angrenzenden Schichten $R_{1}$ und $R_{2}$ über den Querverweis in Bild (B) und (D). (VMO: M. vastus medialis obliquus)

\subsubsection{VMO-Faserverlaufswinkel}

Um den Faserverlaufswinkel des VMO zu bestimmen wurde die sagittale Serie der MRT-Bildgebung verwendet. Es wird die Ebene aufgesucht, in der die Patella vollständig und in ihrer längsten Ausbreitung zu sehen ist.

In diesem Bild (Abb. 3, Bild A) wird zunächst mittels der Winkelfunktion des Centricity PACS-Programm eine Gerade (a) mittig durch den Femur entlang der Längsachse 
gezogen. Danach wird eine zweite Gerade (b) horizontal zur Bildebene gezogen. Der Winkel $(\alpha)$, den diese beiden Geraden einschließen, wird notiert. Nun wird das sagittale Schnittbild aufgesucht, in dem die schräg verlaufenden VMO-Fasern zu sehen sind. In diesem (Abb. 3, Bild B) wird wiederum die horizontal zur Bildebene verlaufende Gerade (d) eingezeichnet. Im nächsten Schritt wird eine zweite Gerade (c), die den zuvor bestimmten Winkel ( $\alpha$ ) einschließt, in entsprechend gleicher Richtung zu (a), also parallel zu Femurlängsachse, erstellt.

Nun erfolgt die Messung des VMO-Faserverlaufswinkels (Abb. 3, Bild B). Dafür wird eine dritte Gerade (e) parallel zum Faserverlauf des VMO in Richtung der Geraden (c) eingezeichnet. Der Winkel ( $\beta$ ), der von Gerade (c) und (e) eingeschlossen wird, repräsentiert den VMO-Faserverlaufswinkel.

Je größer der Winkel $(\beta)$, desto horizontaler ist der Faserverlauf des VMO in Richtung des patellaren Ansatzes.
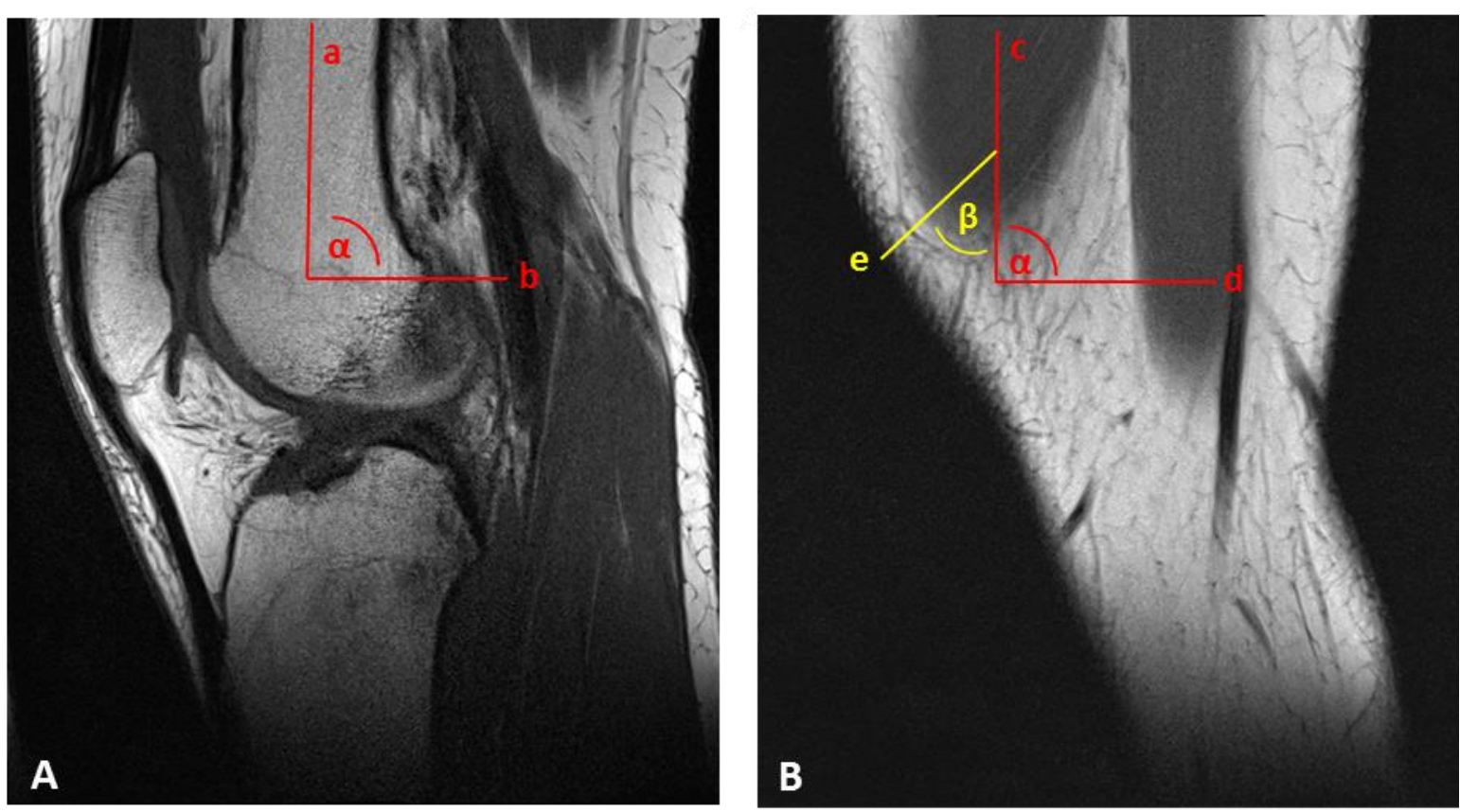

Abbildung 3: Messung des VMO-Faserverlaufwinkels

$\boldsymbol{A}$ zeigt ein Bild der sagittalen Serie. In $\boldsymbol{A}$ wird zunächst eine Gerade a parallel zur Femurlängsachse gezogen. Die Gerade $\boldsymbol{b}$ liegt horizontal zur Bildebene. $\boldsymbol{a}$ und $\boldsymbol{b}$ schließen den Winkel $\boldsymbol{\alpha}$ ein.

$\boldsymbol{B}$ gehört ebenfalls zur sagittalen Serie und zeigt ein Bild des medialen Kniegelenkes und den VMO-Ansatzbereich. Nun wird $\boldsymbol{\alpha}$ auf Bild $\boldsymbol{B}$ übertragen und anschließend die Gerade $\boldsymbol{e}$ parallel zum Faserverlauf des VMO eingezeichnet. $\boldsymbol{c}$ und $\boldsymbol{e}$ schließen den Winkel $\boldsymbol{\beta}$ ein; dieser repräsentiert den VMO-Faserverlaufswinkel. (VMO: M. vastus medialis obliquus)

\subsubsection{Kraniokaudale VMO-Ausdehnung in Relation zur Patella}

Um die Ausdehnung des VMO kraniokaudal im Verhältnis zur Patella zu untersuchen, wird ebenfalls die sagittale Serie der MRT-Untersuchungen verwendet. In der sagittalen Serie wird das Bild genutzt, in dem sich die schrägverlaufenden VMO-Fasern noch ausmachen 
lassen (Abb. 4, Bild A). Dieser kaudalste VMO-Anteil wird markiert $\left(^{*}\right)$, während das sagittale Bild aufgesucht wird, in dem die Patella in voller Länge zu sehen ist (Abb. 4, Bild B). In diesem wird nun vom markierten Punkt $\left(^{*}\right)$ mittels Längenmessfunktion die Strecke (s) zum oberen Patellapol ermittelt. Diese Strecke (s) entspricht der kraniokaudalen Ausdehnung des VMO in Relation zur Patella und somit dem direkten Wirkungsbereich des VMO auf die Patella (Abb. 4, Bild B).
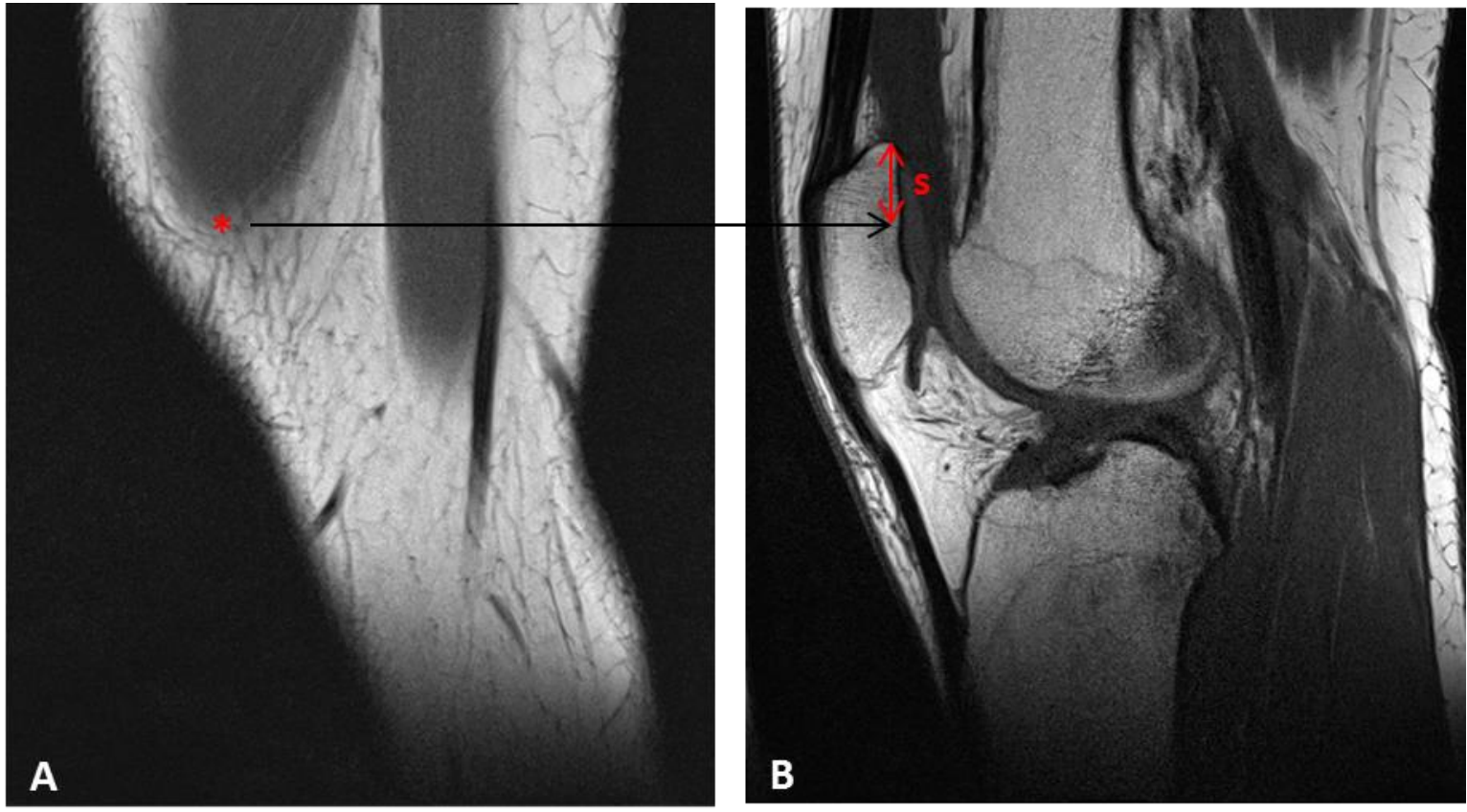

Abbildung 4: Messung der kraniokaudalen VMO-Ausdehnung in Relation zur Patella.

$\boldsymbol{A}$ zeigt ein sagittales Schnittbild des medialen Kniegelenkes in welchem die schrägverlaufenden VMO-Fasern noch zu sehen sind. Der distalste Teil wird durch * markiert. Mit Hilfe des Mauszeigers wird dieser beibehalten, während das sagittale Bild aufgesucht wird, in dem die Patella in voller Länge zu sehen ist (B). Von * wird nun die Strecke s zum oberen Patellapol ermittelt. s repräsentiert die kraniokaudale VMO-Ausdehnung in Relation zur Patella. (VMO: M. vastus medialis obliquus)

\subsection{BAECKE-Score}

Zur Erhebung des individuellen Aktivitätsgrades der einzelnen Patienten wird der BAECKE-Score verwendet.

Dieser besteht aus einem Fragebogen zur physischen Aktivität und ermittelt den Aktivitätsgrad über einen Aktivitäts-Score. Dabei werden drei Indices berechnet: der Arbeits-Index, der Sport-Index und der Freizeit-Index.

Der Fragebogen wurde von BAECKE et al. erstellt und in der Zeitschrift „The American Journal of Clinical Nutriation“ 1982 veröffentlicht.

Für diese Studie wurden die Fragen ins Deutsche übersetzt und in ein patientengerechtes Format gebracht (siehe Anhang 6.4). 


\subsubsection{Aufbau des Fragebogens}

Der BAECKE-Score enthält 16 Parameter, die mittels 16 Fragen erhoben werden. Die Fragen 1-8 (Item $\left.I_{1}-I_{8}\right)$ beziehen sich auf das Arbeitsleben der Einzelnen. Gefragt wird nach dem Berufsbild und nach den physischen Anstrengungen, die die entsprechende Tätigkeit mit sich bringt. Den verschiedenen Berufsgruppen werden dabei Intensitätswerte zwischen 0.76 und $1.76 \mathrm{MJ} / \mathrm{h}$ (1 $\mathrm{MJ}=106$ Joule) zugeordnet. Das niedrigste Intensitätslevel (0.76 MJ/h) haben dabei Berufe, die überwiegend in sitzender Tätigkeit ausgeführt werden (u.a. Lehrer, Sekretärin, Taxifahrer, Studenten). Das mittlere Level (1.26 MJ/h) wird Berufen zugeschrieben, die körperliche Tätigkeiten einschließen (u.a. Handwerker, Fabrikarbeiter, Farmer). Die höchste Intensität (1.76 MJ/h) haben Berufe, bei denen die körperliche Arbeit im Mittelpunkt steht (u.a. Bauarbeiter, Berufe im Sportbereich) Aus $I_{1}-I_{8}$ wird der Arbeits-Index berechnet.

Die Fragen 9-12 ( $\left(I_{9}-I_{12}\right)$ betreffen die sportliche Aktivität. Dabei werden den einzelnen Sportarten wiederum Intensitätswerte zwischen 0.76 und $1.76 \mathrm{MJ} / \mathrm{h}$ zugewiesen. Das niedrigste Intensitätslevel (0.76 MJ/h) wird Aktivitäten wie Segeln, Bowling, Billard und Golf zugeschrieben. Eine mittlere Intensität $(1.26 \mathrm{MJ} / \mathrm{h})$ haben Sportarten wie Radfahren, Badminton, Tanzen, Schwimmen oder Tennis. Sportarten wie Fußball, Boxen, Basketball und andere Ballsportarten entsprechen dem höchsten Intensitätlevel (1.76 MJ/h). Des Weiteren werden die Frequenz und die Regelmäßigkeit des ausgeführten Sports erfragt. Aus den Unterpunkten der Frage 9 ergibt sich der Sport Score $\left(l_{9}\right)$ und aus allen Antworten der Fragen 9-12 berechnete sich der Sport-Index.

Die Fragen 13-16 $\left(\mathrm{I}_{13}-\mathrm{I}_{16}\right)$ ermitteln die Aktivität während der Freizeit. Gefragt wird nach Tätigkeiten im Alltag wie u.a. Fernsehen, Spazierengehen oder Fahrradfahren. Aus diesen Fragen wird der Freizeit-Index bestimmt.

Jede der 16 Fragen ergibt einen bestimmten Punktwert. Aus den einzelnen Werten wiederum berechnen sich die einzelnen Indices.

Die Fragebögen wurden an die Patienten der Fall- wie auch an die der Kontrollgruppe ausgegeben und selbstständig durch die Patienten ausgefüllt.

\subsubsection{Auswertung}

Die Auswertung erfolgte mit Hilfe einer entsprechend erstellten Excel Tabelle.

Kalkulation des Sport-Scores ( $\left.\underline{l}_{9}\right)$ :

$$
\mathbf{I}_{9}=\sum_{i=1}^{2}(\text { Intensität } \times \text { Zeit } \times \text { Verhältnis })
$$


$I_{9}$ wird wiederum ein Wert zwischen 1-5 zugeordnet:

$\begin{array}{ll}I_{9}=\quad & 0 \\ 0,01-<4 & \rightarrow 1 \\ 4-<8 & \rightarrow 2 \\ 8-<12 & \rightarrow 4 \\ >12 & \rightarrow 5\end{array}$

$I_{9}=0 \rightarrow$ Patienten, die keinen Sport machen

\section{Kalkulation der Aktivitäts-Indices:}

$$
\begin{aligned}
& \text { Arbeits-Index }=\left[I_{1}+\left(6-I_{2}\right)+I_{3}+I_{4}+I_{5}+I_{6}+I_{7}+I_{8}\right] \div 8 \\
& \text { Sport-Index }=\left[I_{9}+I_{10}+I_{11}+I_{12}\right] \div 4 \\
& \text { Freizeit-Index }=\left[\left(6-I_{13}\right)+I_{14}+I_{15}+I_{16}\right] \div 4
\end{aligned}
$$

Um den Gesamt-Aktivitäts-Score der einzelnen Patienten zu erhalten, werden die drei Indices addiert. Sie gehen zu gleichen Anteilen in den Aktivitäts-Score ein. Der niedrigste zu erreichende Wert ist dabei 3 (Arbeits- Sport- und Freizeitindex jeweils von 1). Der höchstmögliche Aktivitäts-Score ist 15 (Arbeits-, Sport- und Freizeitindex von jeweils 5). Um die erhobenen Werte bezüglich der VMO-Morphologie zwischen den Patienten vergleichen zu können, muss dieser Parameter für die Aktivität berücksichtigt und im Zusammenhang mit der im MRT gemessenen VMO-Querschnittsfläche und der entsprechenden muskulären Konstitution gesehen werden.

\subsection{Erhebung der anatomischen Hauptparameter der Patellaluxation}

\subsubsection{Bestimmung des TT-TG-Abstandes}

Zur Bestimmung des TT-TG-Abstandes (Tuberositas tibiae-trochlear groove) wurde die Messmethode nach SCHOETTLE et al. (2006) verwendet.

Die TT-TG-Messung erfolgte mittels der sagittalen und der transversalen Serien der MRT-Untersuchung.

Standardisiert wurde zunächst in der sagittalen Serie der Gelenkspalt aufgesucht. Von dort wurde eine $3 \mathrm{~cm}$ lange Linie senkrecht nach kranial gezogen. So erhält man einen einheitlichen Messpunkt $3 \mathrm{~cm}$ oberhalb des Gelenkspalts. Über den Querverweis zur transversalen Serie gelangt man zum entsprechenden axialen Bild. In diesem wird eine erste Gerade senkrecht zur posterioren Kondylenlinie durch den tiefsten Punkt der 
Trochlea gezogen. Danach wird in der transversalen Serie die Tuberositas tibiae aufgesucht und eine zweite Gerade vom vordersten Teil der Tuberositas tibiae parallel zur Trochlealinie gezogen. Die Distanz zwischen diesen beiden Linien entspricht dem TT-TG-Abstand in mm (Abb. 5).

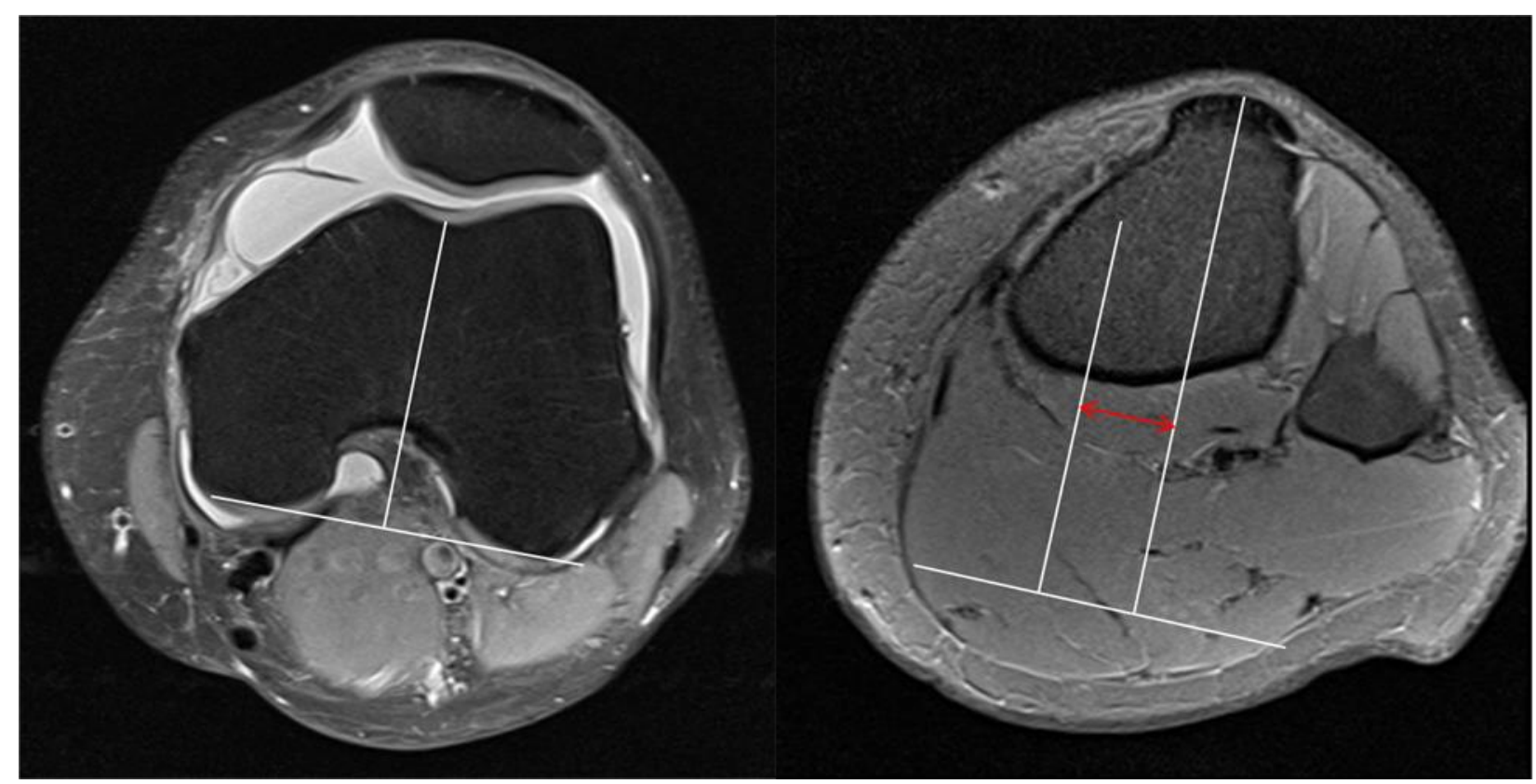

Abbildung 5: Messung des TT-TG-Abstandes.

Eine erste Gerade verbindet die beiden posterioren Kondylen (posteriore Kondylenlinie). Senkrecht zu dieser wird durch den tiefsten Punkt der Trochleagrube eine zweite Linie gezogen. Parallel zu dieser wird eine dritte Linie durch den anteriorsten Punkt der Tuberositas tibiae gezogen. Der Abstand zwischen diesen beiden Linien repräsentiert den TT-TG-Abstand (Pfeil). (TT-TG: Tuberositas tibiae-Trochlea groove)

\subsubsection{Beurteilung der Trochleamorphologie}

Die Beurteilung der Trochlea wurde in dieser Arbeit anhand der Klassifikation der Trochleadysplasie nach DEJOUR (1998) vorgenommen. Dabei erfolgt die Unterteilung entsprechend der Dysplasieschwere in die Typen A bis $D$ (Abb. 6).

Für die Bestimmung des Trochlea-Typs wurde die transversale Bildserie verwendet. In dieser wurde die Form der Trochlea im kranialen Bereich nach DEJOUR (1998) beurteilt und der entsprechende Dysplasie-Typ zugeordnet. 
A

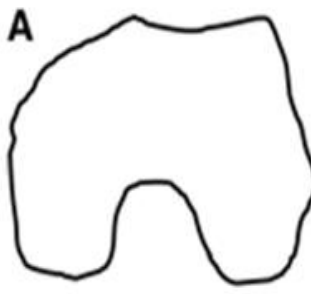

B

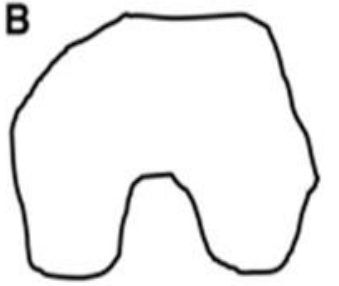

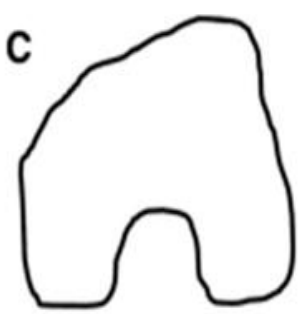

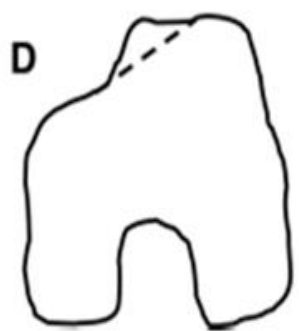

Abbildung 6: Klassifikation der Trochleadysplasie (modifiziert nach DEJOUR et al. 1998, S.1466-1471).

(A) Typ A: Erhaltene Morphologie mit Abflachung der Trochlea

(B) Typ B: Trochlea flach oder beginnend konvex

(C) Typ C: Asymmetrie der Trochlea mit konvexer lateraler Facette und hypoplastischer medialer Facette

(D) Typ D: Asymmetrie der Trochleafacette und „cliff pattern“

Um eine bessere Reliabilität zu erreichen wurde diese 4-Grad-Klassifikation für die vorliegende Studie in eine 2-Grad-Klassifikation umgewandelt (LIPPACHER et al. 2012). Dabei wird zwischen einer low-grade-Dysplasie (Typ A der Dejour-Klassifikation) und einer high-grade-Dysplasie (Typ B-D der Dejour-Klassifikation) differenziert.

\subsubsection{Bestimmung der Patella alta}

Für die Messung der Patellahöhe wurde der Index von INSALL und SALVATI (1971) verwendet (Abb. 7). Die Bestimmung erfolgt mit Hilfe der sagittalen MRT-Serie. In dieser wird das Bild aufgesucht, in dem die Patella in ihrer größten vertikalen Ausdehnung zu sehen ist. In diesem wird zunächst die Länge des Lig. patellae gemessen. Dafür wird mit Hilfe der Längenmessfunktion eine Gerade vom kranialsten Punkt des Lig. patellae am Unterrand der Patella zum tibialen Ansatzpunkt gezogen (Abb. 7, B). Eine weitere Gerade misst diagonal die Länge der Patella (Abb. 7, A) Die Länge der Patellasehne wird durch die Patellalänge dividiert $(B \div A, A b b .7)$. Das Verhältnis von Patellalänge zur Länge des Lig. patellae entspricht dem Index für die Patellahöhe. 


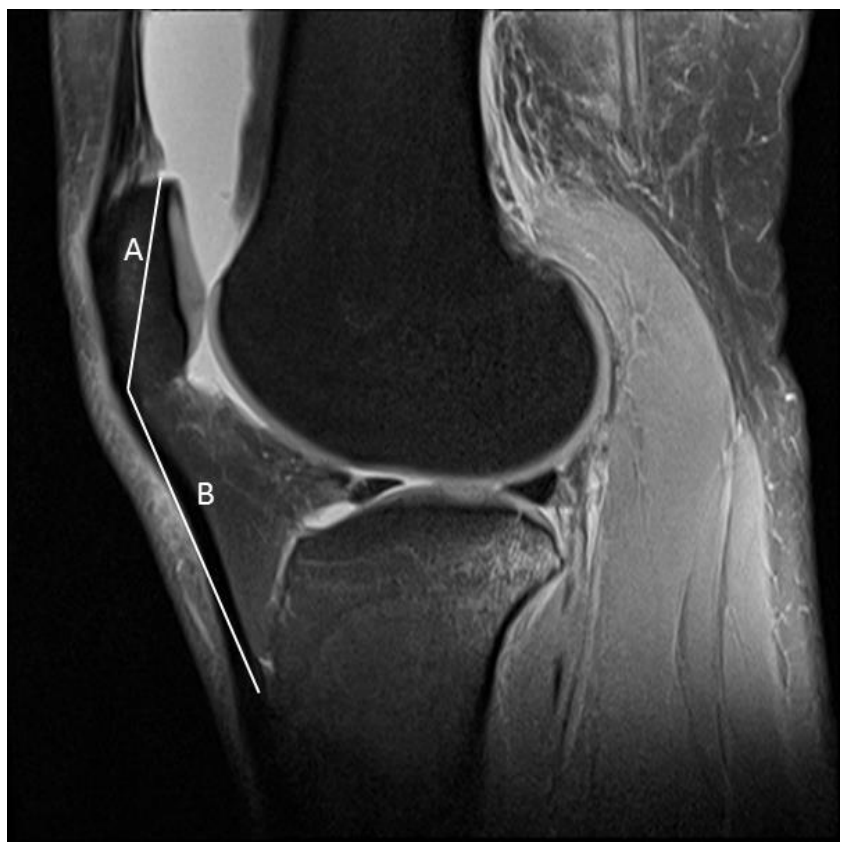

Abbildung 7: Messung der Patellahöhe nach INSALL und SALVATI (1971), S101-104

Eine erste Gerade $\boldsymbol{A}$ entspricht der Patellalänge. Mittels der Gerade $\boldsymbol{B}$ wird die Länge des Lig. patellae ermittelt. Die Länge von $\boldsymbol{B}$ wird dividiert durch die Länge von $\boldsymbol{A}(\boldsymbol{B} \div \boldsymbol{A})$. Der Wert entspricht dem Index nach INSALL und SALVATI.

\subsection{Statistische Auswertung}

Die Daten sind dargestellt als Mittelwert mit Standardabweichung bzw. Median mit Minimum und Maximum. Für die Beurteilung des kategorischen Werts wurde Fisher's exact test genutzt. Der ungepaarte T-Test wurde für den Vergleich der Mittelwerte verwendet.

Eine Power-Analyse der ersten 8 Patienten der Studien- und der Kontrollgruppe (Frauen/Männer jeweils 4/4) wurde durchgeführt. Dabei zeigte sich, dass, um eine Teststärke (Power) (1- $\beta$ Fehler Wahrscheinlichkeit) von $90 \%$ zu erreichen, ein Minimum von 81 Probanden erforderlich ist. Folglich wurden 82 Studienpatienten in die Untersuchungen eingeschlossen. Eine one-way analysis of variance (ANOVA) gefolgt von Dunnett's-Test wurde anschließend für den Vergleich der Studiengruppe und der Kontrollgruppe genutzt.

Um die Intra- und Intertester Reliabilität zu prüfen, wurde bei 15 zufällig ausgewählten MRT-Untersuchungen von Patienten der Studie eine zweite Bestimmung der Parameter durchgeführt. Diese erfolgte entweder durch wiederholte Messung durch denselben Untersucher mit einem mindestens zweiwöchigen Abstand zwischen den Messungen oder durch einen zweiten unabhängigen Untersucher. Die Reliabilität wurde durch das Nutzen der Korrelation (Pearson $r$ ) zwischen den zwei Messungsserien oder der mittleren Differenz (T-Test) zwischen den Untersuchungen beurteilt. 
Alle statistischen Auswertungen erfolgten mit Hilfe des GraphPad Prism® (Version 4, GraphPad Software Inc., San Diego, CA, USA, 2003). Ein p-Wert $<0,05$ wurde als signifikant angenommen.

\subsection{Verwendete Software}

Tabelle 1: Verwendete Software

\begin{tabular}{ll}
\hline Software & Firma \\
\hline Adobe Photoshop 7.0 & Adobe Systems \\
Centricity PACS & GE Healthcare \\
GraphPad Prism ${ }_{\circledast}($ Version 4.00) & GraphPad Software, Inc. \\
Microsoft $_{\circledast}$ Office 2010 & Microsoft Corporation \\
\hline
\end{tabular}




\section{Ergebnisse}

\subsection{Demographische Daten des Patientenkollektivs}

Insgesamt nahmen 82 Patienten, darunter 41 Frauen und 41 Männer, an dieser Studie teil. Die untersuchten Patienten wurden ihrem Krankheitsbild entsprechend den zwei Studiengruppen und der Kontrollgruppe zugeordnet.

Von den 82 Patienten gehörten 30 Patienten in die Gruppe der akuten Erstluxationen (AE). Ebenfalls 30 Patienten litten unter chronischer Instabilität und bildeten somit die Gruppe der Chronischen Luxationen (CL). Die Kontrollgruppe $\mathrm{K}$ besteht aus 22 Patienten.

Bei der Auswahl der Patienten wurde auf ein ausgeglichenes Geschlechterverhältnis geachtet. Je $50 \%$ des Patientenkollektivs waren weiblich. Gruppe AE und CL unterschieden sich demnach im Vergleich zur Kontrollgruppe nicht signifikant in Bezug auf das Geschlecht.

Im Mittel betrug das Alter der Patienten, die an der Studie teilnahmen, 21 Jahre (11-34 Jahre), wobei sich die Gruppen AE und K signifikant ( $p<0,01$ - siehe Tab.2), die Gruppen $C L$ und $K$ nicht signifikant $(p>0,05)$ auf das Alter bezogen unterschieden.

Der Altersdurchschnitt der Gruppe AE betrug 19 Jahre, der in Gruppe CL 21 Jahre und der in Gruppe K 23 Jahre.

Der erhobene Body Mass Index (BMI) lag in der Gruppe AE bei 23,4 kg/m². Im Vergleich dazu war der ermittelte BMI bei einem Wert von $22,7 \mathrm{~kg} / \mathrm{m}^{2}$ in der Studiengruppe CL etwa $3 \%$ kleiner. Der Vergleich von Studiengruppe AE und der Kontrollgruppe ergab in der Kontrollgruppe mit 24,6 kg/m² einen im Mittel ca. 5 \% höheren BMI.

Dies ergab einen nicht signifikanten Unterschied im Vergleich der Gruppen untereinander (AE vers. $K$; $C L$ vers. $K$ mit $p>0,05$ - siehe Tab. 2).

Der erhobene BAECKE-Score ergab im Mittel einen Wert von 8,25, wobei sich die Gruppen untereinander nicht signifikant unterschieden. Der durchschnittliche BAECKE-Score in der Gruppe AE lag bei 8 und in der Gruppe CL 8,13. In der Kontrollgruppe ergab sich ein Wert von 8,63 (Tab. 2). 
Tabelle 2: Demographische Daten des Patientenkollektivs. Die Daten sind präsentiert als Median mit Minimum und Maximum. (AE: Akute Erstluxation, CL: Chronische Luxation, K: Kontrollgruppe, Min: Minimum, Max: Maximum)

\begin{tabular}{lcccc|c}
\hline & Gruppe AE & Gruppe CL & Gruppe K & p-Wert \\
\hline Anzahl & & $\mathrm{n}=30$ & $\mathrm{n}=30$ & $\mathrm{n}=22$ & \\
weiblich & 15 & 15 & 11 & \\
männlich & 15 & 15 & 11 & \\
\hline \multirow{3}{*}{ Alter (Jahre) } & Median & 19 & 21 & 23 & 0,007 \\
& Min - Max & $14-28$ & $11-33$ & $13-34$ & \\
\hline \multirow{3}{*}{ BMI (kg/m ${ }^{2}$ ) } & Median & 23,4 & 22,7 & 24,6 & 0,175 \\
& Min - Max & $18,4-32,1$ & $17,9-28,4$ & $19,9-35$ & \\
\hline & & & & & \\
BAECKE-Score & Median & 8 & 8,13 & 8,63 & 0,411 \\
& Min - Max & $5,5-11,5$ & $6,5-11,75$ & $5,75-11,0$ & \\
\hline
\end{tabular}

\subsection{VMO-Querschnittsfläche}

Der Vergleich des VMO-Parameters Querschnittsfläche ergab keinen signifikanten Unterschied zwischen den akuten Erstluxationen, den chronischen Luxationspatienten und den Kontrollpatienten (AE vs. K und CL vs. K, Tab. 3 und Abb. 8).

In der Studiengruppe AE ergab sich als Median eine summierte Querschnittsfläche von $1697 \mathrm{~mm}^{2}$. Der Unterschied zur Querschnittsfläche der Kontrollgruppe (1944 mm²) betrug damit etwa $13 \%$ (Tab. 3)

Im Vergleich dazu war die Querschnittsfläche der Studiengruppe CL bei einem mittleren summierten Querschnitt von $1671 \mathrm{~mm}^{2}$ etwa $15 \%$ kleiner als die der Kontrollgruppe.

Es zeigte sich somit kein signifikanter Unterschied zwischen Kontrollgruppe und Studiengruppe $A E$ sowie zwischen Kontrollgruppe und Studiengruppe $C L(p>0,1)$. Das Minimum aller gemessenen summierten Werte fand sich mit einer Fläche von $649 \mathrm{~mm}^{2}$ in der Gruppe CL; das Maximum mit einer Fläche von 3460 mm² in der Gruppe AE.

In Gruppe CL ist demnach verglichen mit Gruppe AE und der Kontrollgruppe nur ein leichter Trend zur kleineren Querschnittsfläche zu beobachten. 
Tabelle 3: VMO-Querschnittsfläche. Die Daten sind präsentiert als Median mit Minimum und Maximum. (VMO-QF: VMO-Querschnittsfläche, $95 \%$ Cl: $95 \%$ Konfidenzintervall, AE: Akute Erstluxation, CL: Chronische Luxation, K: Kontrollgruppe)

\begin{tabular}{|c|c|c|c|c|c|}
\hline & $\begin{array}{c}\text { Gruppe AE } \\
n=30\end{array}$ & $\begin{array}{c}\text { Gruppe } \mathrm{CL} \\
\mathrm{n}=30\end{array}$ & $\begin{array}{c}\text { Gruppe } K \\
n=22\end{array}$ & p-Wert & $95 \% \mathrm{Cl}$ \\
\hline VMO-QF $\left(\mathrm{mm}^{2}\right)$ & & & & & $\begin{array}{c}\text { AE vs } \mathrm{K} \\
-64,76-208,4\end{array}$ \\
\hline Median & 674 & 589,5 & 670 & 0,094 & CL vs K \\
\hline Min - Max & $303-1200$ & $251-960$ & $262-1126$ & & $-3,296-269,9$ \\
\hline \multicolumn{6}{|l|}{ VMO-QF $\left(\mathrm{mm}^{2}\right)$ - summiert } \\
\hline Median & 1697 & 1671 & 1944 & $>0,1$ & \\
\hline Min - Max & $842-3460$ & $649-2724$ & $687-3227$ & & \\
\hline
\end{tabular}

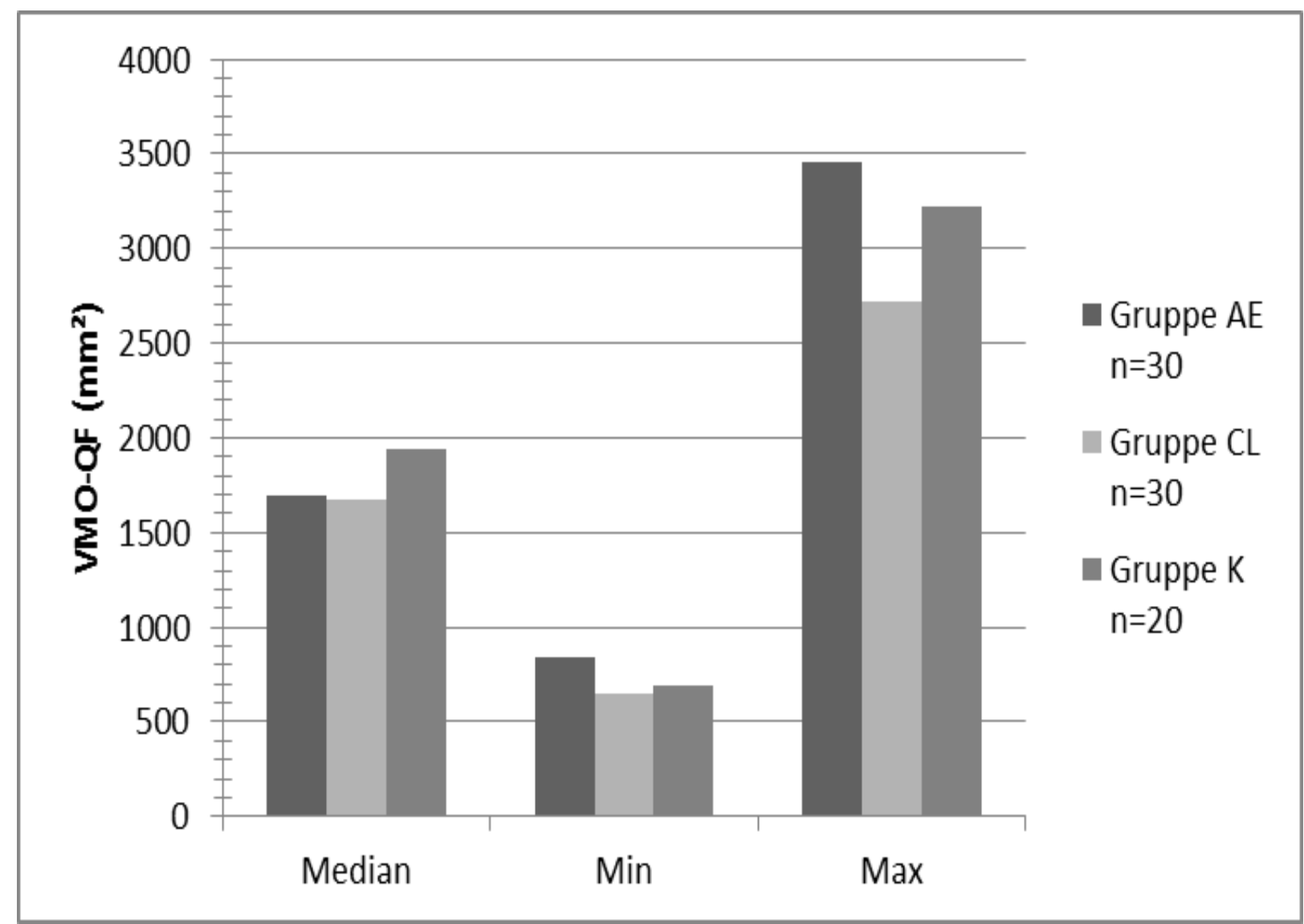

Abbildung 8: Darstellung der VMO-Querschnittsfläche. Die Daten sind präsentiert als Median mit Minimum und Maximum. (VMO-QF: VMO-Querschnittsfläche, AE: Akute Erstluxation, CL: Chronische Luxation, K: Kontrollgruppe, Min: Minimum, Max: Maximum) 


\subsection{VMO-Faserverlaufswinkel}

Bei der Auswertung des VMO-Faserverlaufswinkels und dem Vergleich der Gruppen ergab sich eine nicht signifikante Differenz der Gruppen (AE vs. K und CL vs. K; Tab. 4 und Abb. 9) untereinander ( $p>0,186)$. Lediglich in Gruppe $C L$ ist ein geringer Trend zu einem kleineren Winkel zu beobachten.

Der Faserwinkel betrug in der Studiengruppe AE im Median $47^{\circ}$. Die Kontrollgruppe wies hingegen einen um $1^{\circ}$ horizontaler verlaufenden Faserwinkel auf. In Studiengruppe CL lag der Faserverlaufswinkel im Mittel bei $43,5^{\circ}$. Dies ergibt einen etwa $4,5^{\circ}$ steileren Faserverlauf im Vergleich zur Kontrollgruppe.

Tabelle 4: VMO-Faserverlaufswinkel. Die Daten sind präsentiert als Median mit Minimum und Maximum. (VMO-FW: VMO-Faserverlaufswinkel, $95 \% \mathrm{Cl}$ : $95 \%$ Konfidenzintervall, AE: Akute Erstluxation, CL: Chronische Luxation, K: Kontrollgruppe, Min: Minimum, Max: Maximum)

\begin{tabular}{cccc|c|c}
\hline & Gruppe AE & Gruppe CL & Gruppe K & p-Wert & 95\% Cl \\
\hline VMO-FW ( $\left.{ }^{\circ}\right)$ & $\mathrm{n}=30$ & $\mathrm{n}=30$ & $\mathrm{n}=22$ & & AE vs K \\
Median & 47 & 43,5 & 48 & 0,186 & CL vs K \\
Min-Max & $30-59$ & $32-60$ & $36-64$ & & $-0,9585-8,858$ \\
\hline
\end{tabular}

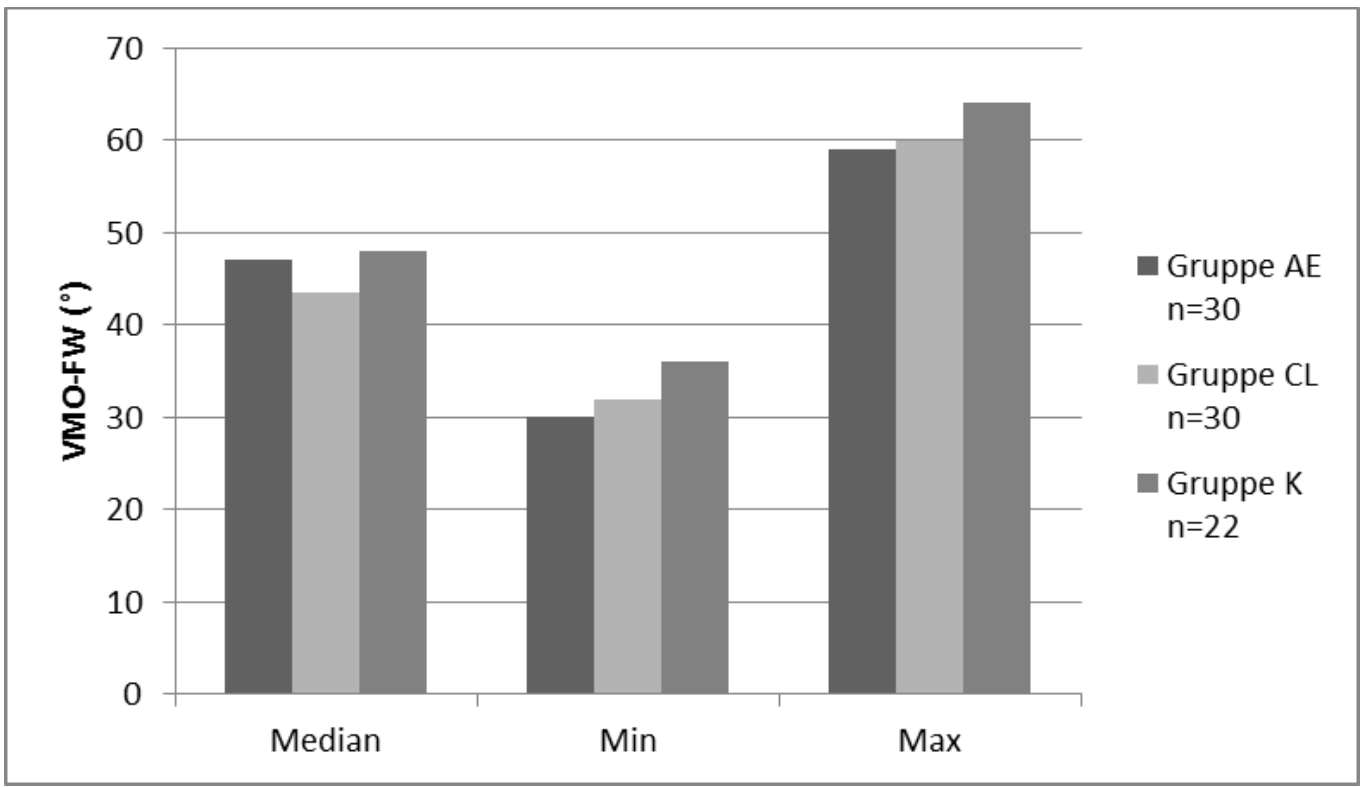

Abbildung 9: Darstellung des VMO-Faserverlaufswinkels. Die Daten sind präsentiert als Median mit Minimum und Maximum. (VMO-FW: VMO-Faserverlaufswinkel, AE: Akute Erstluxation, CL: Chronische Luxation, K: Kontrollgruppe, Min: Minimum, Max: Maximum) 


\subsection{Kraniokaudale VMO-Ausdehnung in Relation zur Patella}

Der Vergleich der Studiengruppen untereinander in Bezug auf die kraniokaudale VMO-Ausdehnung ergab keinen signifikanten Unterschied ( $p>0,05$ für AE vs. K; CL vs. K; Tab. 5 und Abb. 10). In allen Studiengruppen lag der Wert bei ungefähr 13,5 mm.

Der Vergleich der Studiengruppe AE und der Kontrollgruppe ergab eine Differenz von im Mittel 0,5 mm und war somit in der Kontrollgruppe 3,8\% kleiner als in der Studiengruppe $\mathrm{AE}$.

Die Studiengruppe CL unterschied sich von der Kontrollgruppe bezüglich ihrer kraniokaudalen VMO-Ausdehnung nur um 1,5\%.

Tabelle 5: Kraniokaudale Ausdehnung des VMO in Relation zur Patella. Die Daten sind präsentiert und als Median mit Minimum und Maximum. (VMO-KkA: VMO-Kraniokaudale Ausdehnung, $95 \% \mathrm{Cl}$ : $95 \%$ Konfidenzintervall, AE: Akute Erstluxation, CL: Chronische Luxation, K: Kontrollgruppe).

\begin{tabular}{rccc|c|c}
\hline & Gruppe AE & Gruppe CL & Gruppe K & p-Wert & 95\% Cl \\
\hline & $\mathrm{n}=30$ & $\mathrm{n}=30$ & $\mathrm{n}=22$ & & AE vs K \\
VMO-KkA (mm) & & & & & $-2,46-3,197$ \\
Median & 13,8 & 13,5 & 13,3 & 0,957 & CL vs K \\
Min - Max & $6-30$ & $5-22,5$ & $9,8-20$ & & $-2,576-3,08$ \\
\hline
\end{tabular}

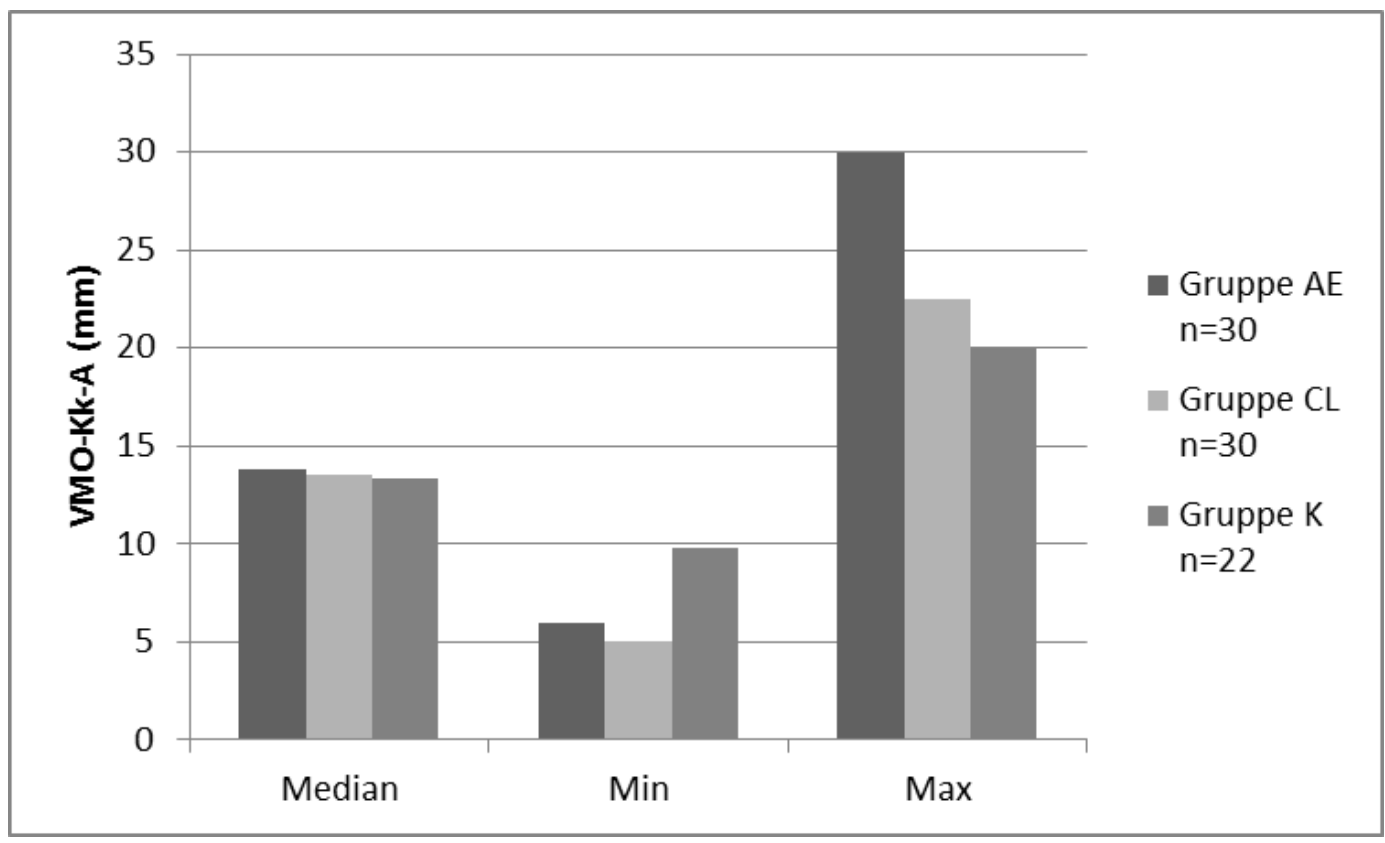

Abbildung 10: Darstellung der kraniokaudalen VMO-Ausdehnung. Die Daten sind präsentiert als Median mit Minimum und Maximum. (VMO-KkA: VMO-Kraniokaudale Ausdehnung, AE: Akute Erstluxation, CL: Chronische Luxation, K: Kontrollgruppe, Min: Minimum, Max: Maximum) 


\subsection{Anatomische Hauptparameter der Patellaluxation}

Die anatomischen Hauptprädispositionsfaktoren der patellofemoralen Instabilität umfassen die Trochleadysplasie, die Patella alta und einen veränderten TT-TG-Abstand. Im Vergleich zur Kontrollgruppe zeigten sowohl Patienten der Gruppe AE mit akuten Erstluxationen wie auch die Gruppe CL der chronischen Luxationspatienten das typische Risikoprofil der lateralen Patellaluxation mit einem vergrößerten TT-TG-Abstand, einer dysplastischen Trochlea und einer Patella alta (Tab. 6).

Tabelle 6: Vergleich der anatomischen Hauptparameter Trochleadysplasie, TT-TG-Abstand und Patellahöhe. Darstellung dieser Faktoren bei akuter Erstluxation, rezidivierenden Luxationen und Kontrollpatienten. Die Daten sind präsentiert als Häufigkeit und als Mittelwert mit Standardabweichung. (AE: Akute Erstluxation, CL: Chronische Luxation, K: Kontrollgruppe, TT-TG: Tuberositas tibiae-Trochlea groove, SD: Standardabweichung - standard deviation).

\begin{tabular}{rccc|c}
\hline & $\begin{array}{c}\text { Gruppe AE } \\
\mathrm{n}=30\end{array}$ & $\begin{array}{c}\text { Gruppe CL } \\
\mathrm{n}=30\end{array}$ & $\begin{array}{c}\text { Gruppe K } \\
\mathrm{n}=22\end{array}$ & p-Wert \\
\hline Trochleadysplasie & 1 & & & \\
keine & 9 & 0 & 16 & \\
high-grade & 20 & 4 & 5 & $<0,001$ \\
\hline TT-TG-Abstand (mm) & 26 & 0 & $<0,01$ \\
Mittelwert mit SD & $13,6 \pm 3,3$ & $16,1 \pm 4,1$ & $9,0 \pm 3,7$ & \\
\hline Patellahöhe & & & $1,15 \pm 0,11$ & $<0,0053$ \\
Mittelwert mit SD & $1,27 \pm 0,17$ & $1,29 \pm 0,17$ & &
\end{tabular}

\subsubsection{Trochleadysplasie}

In der Gruppe der akuten Erstluxationen besteht bei etwa $67 \%$ der Patienten eine high-grade-Trochleadysplasie. Knapp ein weiteres Drittel weist eine low-grade-Dysplasie auf.

In der Gruppe CL haben 87 \% der Patienten eine high-grade-Dysplasie, während bei etwa $76 \%$ der Patienten der Kontrollgruppe eine normale Trochleaanatomie besteht. Dies ergibt ein Signifikanzniveau von $p<0,001$ (Abb. 11). 


\section{Trochleadysplasie Häufigkeitsverteilung}
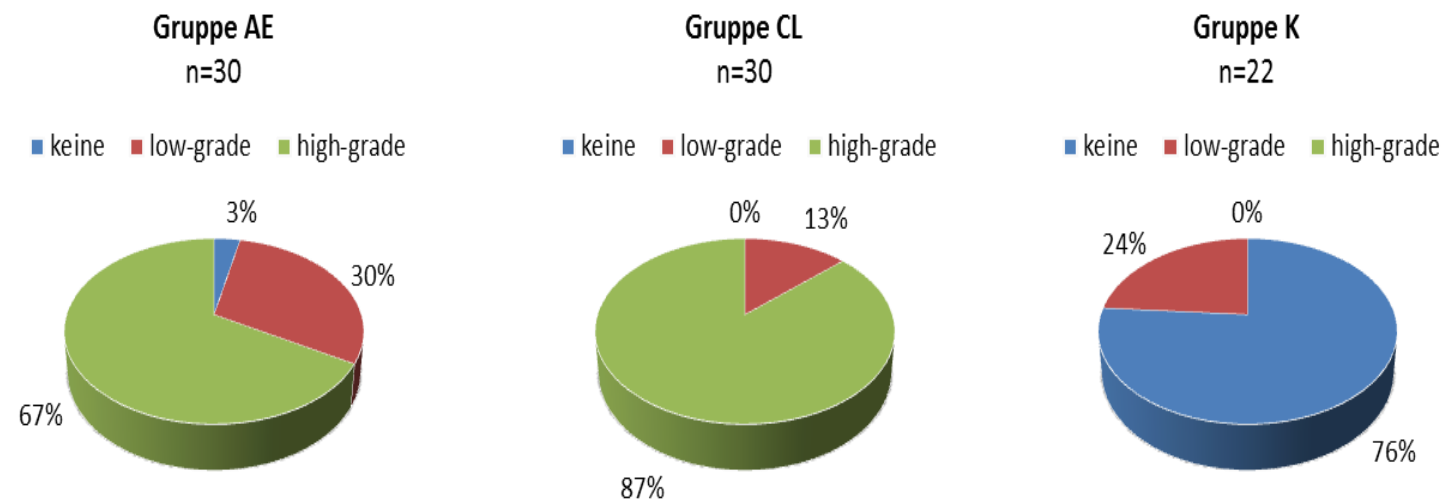

Abbildung 11: Darstellung der Häufigkeit der Trochleadysplasie in den Studiengruppen. Die Daten sind präsentiert als Häufigkeit in \%. (AE: Akute Erstluxation, CL: Chronische Luxation, K: Kontrollgruppe)

\subsubsection{TT-TG-Abstand}

Die Studiengruppen AE und CL weisen im Vergleich zu der Kontrollgruppe einen signifikant größeren TT-TG-Abstand auf $(p<0,01)$. Während in der Kontrollgruppe der Mittelwert bei 9 mm liegt, ist der Abstand in Gruppe AE etwa 34 \% und in Gruppe CL 45 \% größer (Abb. 12).

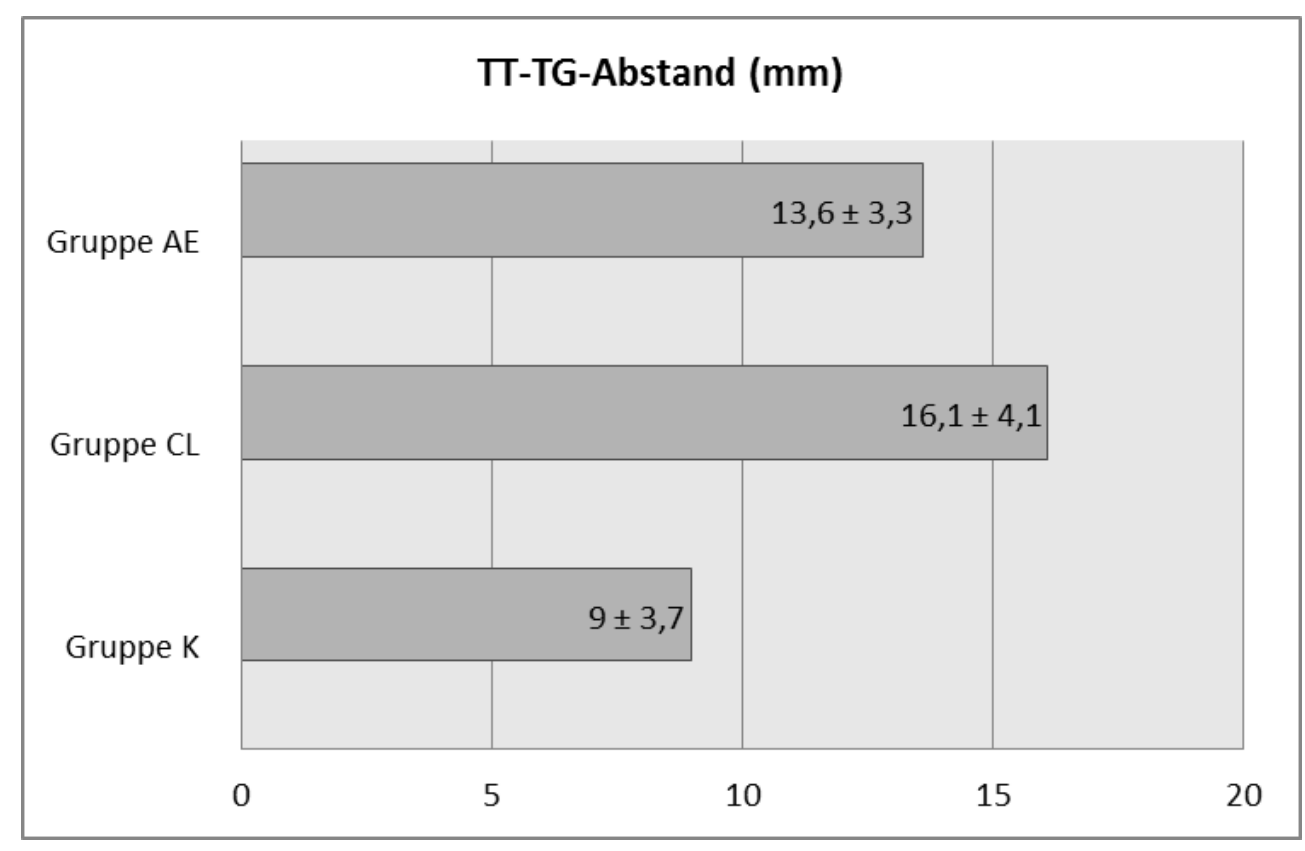

Abbildung 12: Darstellung des TT-TG-Abstandes $(\mathrm{mm})$ der Studiengruppen im Vergleich zu der Kontrollgruppe. Die Daten sind präsentiert als Mittelwerte mit Standardabweichung. (AE: Akute Erstluxation, $\mathrm{CL}$ : Chronische Luxation, K: Kontrollgruppe) 


\subsubsection{Patellahöhe}

Beim Vergleich der Kontrollgruppe mit den Studiengruppen AE und CL zeigt sich in Bezug auf die Patellahöhe ebenfalls eine signifikante Differenz $(p<0,0053)$. In der Kontrollgruppe lag der Index für die Patellahöhe durchschnittlich bei 1,15 $\pm 0,11$, während sich in der Gruppe AE im Mittel $10 \%$ und in der Gruppe CL $12 \%$ höhere Werte ergaben (Abb. 13).

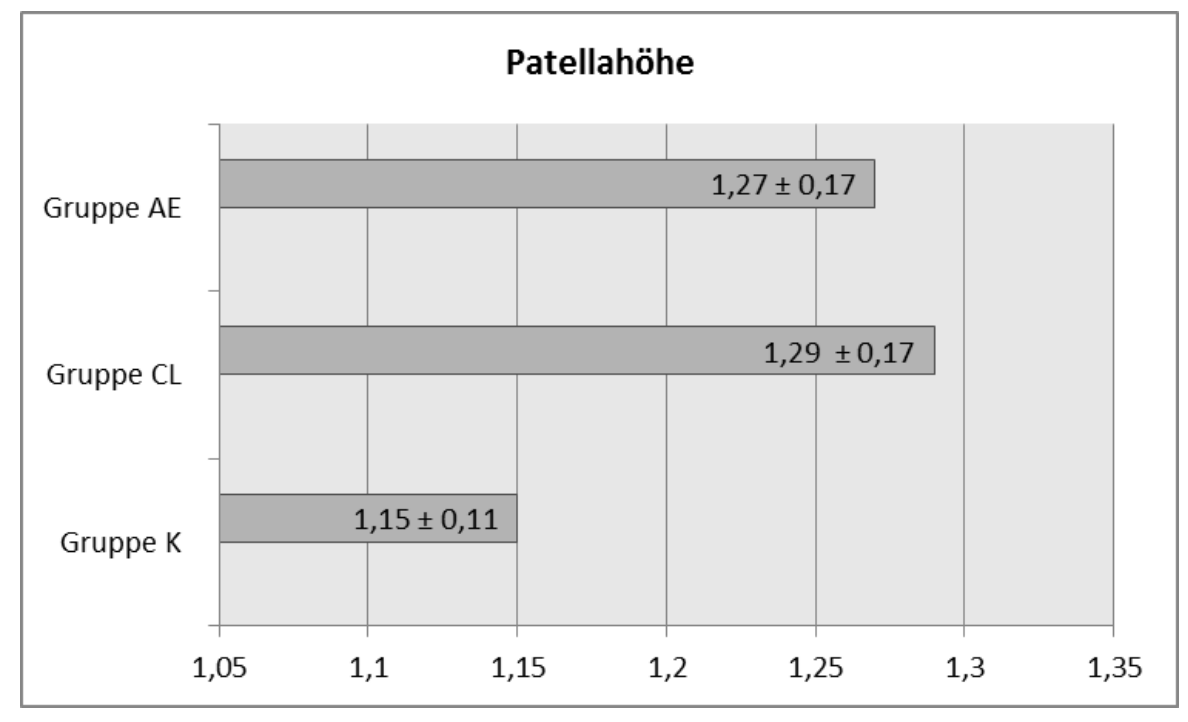

Abbildung 13: Darstellung der Patellahöhe. Die Daten sind präsentiert als Mittelwerte mit Standardabweichung. (AE: Akute Erstluxation, CL: Chronische Luxation, K: Kontrollgruppe) 


\subsection{Intra- und Intertester-Reliabilität}

Die Intra- und Intertester-Reliabilität dieser Studie zeigt eine hohe Korrelation in Bezug auf die drei gemessenen Parameter. Es wurde keine signifikante Differenz zwischen den Messungsserien beobachtet (Tab. 7).

Tabelle 7: Intra- und Intertester-Reliabilität. Korrelation und Durchschnitt der Messungsdifferenzen zwischen den zwei Messungsserien derselben 15 Patienten; durchgeführt von einem Untersucher und zwei verschiedenen Untersuchern. (VMO-QF: VMO-Querschnittsfläche, VMO-FW: VMO-Faserverlaufswinkel, VMO-KkA: VMO-Kraniokaudale Ausdehnung)

\begin{tabular}{lcccc}
\hline & Pearson $\mathbf{r}$ & p-Wert & Mittelwert & p-Wert \\
\hline Intratester-Reliabilität & & & & \\
VMO-QF & 0,99 & $<0,0001$ & 5,58 & 0,36 \\
VMO-FW & 0,97 & $<0,0001$ & 0,1 & 0,9 \\
VMO-KkA & 0,97 & $<0,0001$ & 0,17 & 0,71 \\
\hline Intertester-Reliabilität & & & & \\
VMO-QF & 0,99 & $<0,0001$ & 18,17 & 0,1 \\
VMO-FW & 0,84 & 0,0003 & 0,56 & 0,72 \\
VMO-KkA & 0,92 & 0,0001 & $-0,33$ & 0,44 \\
\hline
\end{tabular}




\section{Diskussion}

Die patellofemorale Instabilität ist ein im klinischen Alltag stark präsentes Krankheitsbild und daher in den letzten Jahren verstärkt ins wissenschaftliche Interesse gerückt. Verschiedene Prädispositionsfaktoren wurden bereits genau untersucht und beschrieben. Die Bedeutung der muskulären dynamischen Stabilisatoren, insbesondere die Rolle des VMO, blieb dabei unklar und wurde in der Literatur kontrovers diskutiert.

Ziel der vorliegenden Studie war die Untersuchung der morphologischen Parameter des VMO und deren Wert für die Aufrechterhaltung der patellofemoralen Stabilität im Kontext der klinischen und anatomischen Situation der primären und chronischen Patellaluxation.

\subsection{Demographische Daten des Patientenkollektivs}

Um die Studiengruppen untereinander vergleichbar machen zu können, wurden die Parameter Alter, Geschlecht, BMI und physische Aktivität erhoben und die Gruppen untereinander in ihren Werten angeglichen.

Die Studiengruppe AE und die Kontrollgruppe unterschieden sich zwar signifikant in Bezug auf das Alter, jedoch gehe ich davon aus, dass dieser Unterschied keinen relevanten Einfluss auf die Ergebnisse gehabt haben dürfe. Verschiedene Studien zeigen zwar, dass es zu einer Reduktion der Muskelmasse mit zunehmendem Alter kommt (FRONTERA et al. 1991, COHN et al. 1980, BERRY et al. 2008), jedoch lag die mittlere Differenz unserer Gruppen bei lediglich 4 Jahren. Im Hinblick auf unsere Studie und die erhobenen VMO-Parameter, v.a. die VMO-Querschnittsfläche, ist dieser Aspekt bezogen auf die Vergleichbarkeit der Gruppen daher eher zu vernachlässigen. Das Altersmaximum in dieser Studie lag bei 34 Jahren. Das durchschnittliche Alter betrug 21 Jahre. In dieser Altersspanne kommt es eher nicht zu gravierenden altersbedingten Veränderungen der muskulären Konstitution. Alle Studienpatienten befinden sich in einem Alter, in dem die muskuläre Situation vergleichbar sein sollte. Daher ist für die Studie diesbezüglich nicht von einer Beeinflussung der Ergebnisse bedingt durch den Altersunterschied zwischen der Studiengruppe AE und der Kontrollgruppe auszugehen.

BERRY et al. (2008) konnten außerdem zeigen, dass das weibliche Geschlecht mit einer kleineren Querschnittsfläche des M. vastus medialis und M. vastus lateralis assoziiert ist. Weitere Studien stützen dies und zeigten, dass Frauen im Mittel weniger Muskelmasse im Verhältnis zu Männern haben (FRONTERA et al. 1991, VANDERVOORT und McCOMAS 1986).

In der vorliegenden Untersuchung wurde daher auf ein exakt ausgeglichenes Geschlechterverhältnis geachtet. 
Der Body Mass Index (BMI) korreliert ebenfalls mit der Muskelquerschnittsfläche. Ein kleiner BMl ist assoziiert mit einer geringeren muskulären Querschnittsfläche (BERRY et al. 2008). Ein hoher BMl ist bedingt durch die größere Körpermasse mit einer größeren Muskelquerschnittsfläche vergesellschaftet.

Die physische Aktivität des Menschen spielt ebenso eine entscheidende Rolle für die muskuläre Konstitution. BERRY et al. (2008) konnten in ihren Untersuchungen nachweisen, dass regelmäßige sportliche Aktivität positiv mit der Querschnittsfläche korreliert. Die physische Aktivität der Patienten dieser Studie wurde mittels BAECKE-Score objektiviert und somit in den Gruppen untereinander angeglichen.

Da es in dieser Studie keine signifikanten Unterschiede in Bezug auf die erhobenen demographischen Daten, mit Ausnahme des Alters, zwischen den Studiengruppen AE und $\mathrm{CL}$ sowie der Kontrollgruppe untereinander gab, ist die Vergleichbarkeit für die untersuchten VMO-Parameter gegeben.

\subsection{VMO-Parameter}

Das Hauptergebnis dieser Studie ist, dass sich im Hinblick auf die Morphologie des VMO kein signifikanter Unterschied bei Patienten mit akuten Erstluxationen und chronischen Luxationen verglichen mit einer asymptomatischen Kontrollgruppe gezeigt hat. Gleichzeitig lassen die Ergebnisse vermuten, dass ein normal konstituierter VMO-Muskel nicht in der Lage ist, die patellofemorale Stabilität zu erhalten bzw. bei zugrundeliegenden prädisponierenden anatomischen Faktoren akute und chronische Patellaluxationen zu verhindern. Interessant ist dies, da dem VMO in der Literatur mehrfach eine wichtige Rolle in der Pathophysiologie der patellofemoralen Instabilität zugeschrieben wurde (FAHRAMAND et al. 1998, GILLEARD et al. 1998). Parallel dazu wurde häufig die Rehabilitation der Quadrizepskraft und insbesondere die Kräftigung des VMO als zwingendes Ziel angesehen, um einem patellofemoralem Maltracking entgegenzuwirken (LIN et al. 2008, PAL et al. 2012).

Auch wenn in mehreren anatomischen und biomechanischen In-Vitro-Studien der Stellenwert des VMO-Muskels als dynamischer Stabilisator des Patellofemoralgelenks hervorgehoben wurde (FARAHMAND et al. 1998, SAKAI et al. 2000, SENAVONGSE und AMIS 2005, PHILIPPOT et al. 2012), fehlt weiterhin eine evidenzbasierte Aussage bezüglich seines eigentlichen stabilisierenden Effekts unter klinischen Verhältnissen.

SAKAI et al. (2000) konnten in ihrer Studie mit Hilfe von Kadaverknien nachweisen, dass eine simulierte VMO-Schwächung einen signifikanten lateralen Patellashift zwischen 0-15 Knieflexion bewirkt. Weitere Kadaverstudien konnten zeigen, dass die Patella sich bei 
Reduktion der VMO-Spannung nach lateral verschiebt (BOSE et al. 1980, LIEB und PERRY 1968).

ELIAS und WHITE veröffentlichten 2004, dass es die wichtigste Aufgabe des VMO sei, einen dynamischen Widerstand zu bilden, um so der natürlichen Tendenz der Patella nach lateral entgegenzuwirken. Gleichzeitig konnten SENAVONGSE und AMIS (2005) in ihren Untersuchungen zeigen, dass eine Relaxation des $\mathrm{VMO}$ zwischen $20^{\circ}$ und $90^{\circ}$ Flexion eine mittlere Reduktion der lateralen patellofemoralen Stabilität von etwa $30 \%$ verursacht und obwohl die Patella im gestreckten Knie am wenigsten stabil ist, dokumentierten sie in diesem Bereich nur eine Reduktion der Stabilität von ca. $14 \%$. Durch SENAVONGSE und AMIS konnte der VMO daher in vitro nicht als wichtigster Stabilisator der Patella identifiziert werden. Da sich in unserer Untersuchung bei allen drei gemessenen VMO-Parametern kein signifikanter Unterschied zwischen den asymptomatischen Kontrollpatienten und den Luxationspatienten zeigte, unterstützen die klinischen Ergebnisse der vorliegenden Studie erstmals die oben genannten und in vitro gewonnenen Erkenntnisse.

Bezogen auf die gemessene VMO-Querschnittsfläche konnte lediglich ein Trend zum kleineren Querschnitt bei den Studiengruppen beobachtet werden.

Grundsätzlich ist die Querschnittsfläche eines Muskels von verschiedenen Faktoren abhängig. Körperliche Schonung, ein niedriger BMI und hohes Alter sind mit einer kleineren Querschnittsfläche assoziiert, während physische Aktivität und ein hoher BMI mit einer größeren Querschnittsfläche verbunden sind.

BERRY et al. (2008) konnten diese positive Korrelation von körperlicher Aktivität und Querschnittsfläche des VMO nachweisen und schlussfolgerten, dass eine große VMO-Querschnittsfläche der Lateralisierung der Patella entgegenwirke und daher günstige Auswirkungen auf die biomechanische Situation des Patellofemoralgelenks habe. BAMMAN et al. (2000) untersuchten außerdem den Zusammenhang zwischen Querschnittsfläche und Muskelkraft. Sie konnten dabei belegen, dass die Muskel-Querschnittsfläche proportional zur Muskelkraft ist. Anzunehmen ist, dass somit ein Zusammenhang zwischen physischer Aktivität, Querschnittsfläche und Kraftentwicklung besteht und letztlich von der Ausprägung des Muskels auch die muskelgeführte gelenkstabilisierende Wirkung beeinflusst wird (MITSIOPOULOS et al. 1998, BERRY et al. 2008, PATTYN et al. 2011).

In einer Kadaverstudie von RAIMONDO et al. (1998) ergab sich durchschnittlich eine VMO-Querschnittsfläche von $310( \pm 130) \mathrm{mm}^{2}$. In unseren Untersuchungen zeigten sich hingegen mit einer mittleren Querschnittsfläche am Referenzpunkt $R$ in der Gruppe AE von $674 \mathrm{~mm}^{2}$, in der Gruppe CL von 589,5 $\mathrm{mm}^{2}$ und in der Kontrollgruppe von $670 \mathrm{~mm}^{2}$ insgesamt höhere Werte. Dieser relativ große Unterschied ist wahrscheinlich durch 
unterschiedliche Messpunkte begründet. Als Referenzpunkt R diente in der vorliegenden Studie der obere Patellapol. Je distaler die Messung der Querschnittsfläche erfolgt, desto kleinere Werte ergeben sich für die VMO-Querschnittsfläche.

Die Patienten mit chronischer Luxationssymptomatik wiesen in unserer Studie verglichen mit der asymptomatischen Kontrollgruppe im Mittel eine um etwa $15 \%$ reduzierte Querschnittsfläche auf. Die Patienten mit akuter Erstluxation verfügten über eine um etwa $13 \%$ kleinere Querschnittsfläche.

Fraglich ist, ob diese Tendenz zum kleineren Querschnitt bei den Patienten mit Luxationsereignis bereits als $\mathrm{VMO}-$ Atrophie zu werten ist und ob dies bereits nach der Erstluxation, also durch eine Verletzung des Muskels, zustande kommt oder ob die chronische Instabilität und damit verbunden die körperliche Schonung und veränderte Belastung des betroffenen Beines dem zugrunde liegen.

FOX stellte 1975 in seiner Studie einen Zusammenhang zwischen patellofemoralem Maltracking und VMO-Atrophie beim Patellofemoralen Schmerzsyndrom her. Als Folge des gestörten Patellagleitens wird angenommen, dass es zur Insuffizienz und sekundären Atrophie mit Hypoplasie des VMO kommt. Bezieht man diese Erkenntnisse auf das Krankheitsbild der patellofemoralen Instabilität, so wäre dies eine mögliche Erklärung für den Trend zur kleineren Querschnittsfläche bei den Luxationspatienten. Bei diesen Patienten liegt meist ein Fehlgleiten der Patella bedingt durch u.a. Trochleadysplasie, Patella alta oder einen erhöhten TT-TG-Abstand vor. Dies führt zu der Annahme, dass es infolgedessen zu einer sekundären VMO-Schwäche bedingt durch die Instabilitätsproblematik kommt. Begründet auf der Tatsache, dass der VMO als Teil des M. quadriceps femoris als erstes Kompartiment bei Inaktivität zu Schwächung und Atrophie neigt und als letzter regeneriert (FOX 1975), darf jedoch angenommen werden, dass der beobachtete Trend zur kleineren Querschnittsfläche durch die Bewegungseinschränkung bei rezidivierenden Schmerzepisoden sowie durch die physische Inaktivität nach Luxationsereignissen zustande kommt.

Der VMO ist durch schräg verlaufende Muskelfaserzüge charakterisiert. Von verschiedenen Autoren wird postuliert, dass der VMO aufgrund seiner schräg ziehenden Muskelfasern als medial dynamisch wirkender Stabilisator eine Rolle für das patellofemorale Tracking und die patellofemorale Stabilität spielt (LIEB und PERRY 1968, GOH et al. 1995, HINTON und SHARMA 2003). Je größer der VMO-Faserwinkel, desto horizontaler der Faserverlauf und die mediodorsale Zugrichtung des VMO. Daher ergibt sich für einen größeren Winkel auch eine horizontaler ausgerichtete und von mediodorsal auf die Patella wirkende Zugkraft des VMO. Je nach Autor variieren dabei die Angaben zu den VMO-Faserverlaufswinkeln zwischen $42,5^{\circ}$ und $60^{\circ}$ (FARAHMAND et al. 1998, 
RAIMONDO et al. 1998, SAKAl et al. 2000, HINTON und SHARMA 2003). Diese Daten korrelieren mit den durchschnittlich ermittelten $48^{\circ}$ VMO-Faserwinkel der Kontrollgruppe bzw. den $47^{\circ}$ der Gruppe AE und den $43,5^{\circ}$ der Gruppe CL dieser Studie, ohne dass ein signifikanter Unterschied zwischen den Studiengruppen und der Kontrollgruppe nachgewiesen werden konnte. In der Gruppe der chronischen Luxationspatienten konnte im Vergleich zur Kontrollgruppe ein etwa $4,5^{\circ}$ steilerer Faserverlauf beobachtet werden. Hier ist folglich eine Tendenz zu einem geringeren Faserwinkel in Gruppe CL zu vermerken. Unklar bleibt, ob dieser Unterschied vorbestehend und anlagebedingt ist, oder ob sich der Winkel posttraumatisch verändert hat. Da die Patienten mit akuter Erstluxation und die asymptomatische Kontrollgruppe nahezu identische Faserwinkel aufweisen, kann vermutet werden, dass es erst nach der Luxation, also sekundär, zur Änderung des Winkels kommt. Ausschlaggebend ist diesbezüglich die anatomische und funktionelle Beziehung zwischen MPFL und VMO. Von verschiedenen Autoren wurde diskutiert, dass eine Verletzung des MPFL an seinem femoralen Ursprung von einer Schädigung des VMO begleitet sein kann (SALLAY et al. 1996, DESIO et al. 1998, AHMAD et al. 2000). Es wird geschlussfolgert, dass es durch eine stattgehabte Patellaluxation und die Mitverletzung des VMO zu einem veränderten Faserwinkel und dadurch zu einer veränderten VMO-Zugrichtung und Orientierung der Muskelfasern kommt (SALLAY et al. 1996, ELIAS et al. 2002).

Von AMIS et al. (2003) wurde beschrieben, dass zwischen VMO und MPFL häufig eine Verbindung bestehe und dadurch ein gemeinsamer Ansatzpunkt am medialen Patellarand entstehe. Durch diese Verbindung seien beide Strukturen lagebedingt häufig zusammen verletzt. Bei Ruptur des MPFLs als Folge einer Luxation komme es gleichzeitig zur Schädigung des VMO und zum Zerreißen der Faserverbindung beider Strukturen. Der VMO reiße dabei fortschreitend in proximaler Richtung ein und verliere dadurch seine ursprüngliche Faserorientierung (AHMAD et al. 2000, ELIAS et al. 2002).

Insbesondere für akute Erstluxationen wurde beschrieben, dass die MPFL-Verletzung häufig aus einer Ablösung der distalen VMO-Fasern bzw. des VMO-Ansatzes am Femur resultiere. Daraus ergebe sich dann eine Verlagerung des VMO nach proximal und lateral. Als Konsequenz ergibt sich eine Veränderung der Zugrichtung bzw. des Kraftvektors der VMO-Fasern. Die dorsomediale Zugkraft des VMO auf die Patella kann dadurch bedingt schwächer ausfallen (SALLAY et al. 1996, DESIO et al. 1998, AHMAD et al. 2000).

Festzuhalten ist, dass der schräge Verlauf des VMO aufgrund seiner Biomechanik die mediale Stabilität des Patellofemoralgelenks günstig beeinflussen kann (LIEB und PERRY 1968, GOH et al. 1995, HINTON und SHARMA 2003). Gleichzeitig konnte jedoch in der vorliegenden Studie nur eine leichte Tendenz zum kleineren Winkel und damit weniger horizontal orientierten VMO-Muskelfasern bei chronischen Luxationspatienten gezeigt 
werden, sodass ein sicherer Zusammenhang zwischen patellofemoraler Instabilität und pathologisch veränderten Faserwinkelwerten nicht hergestellt werden kann.

Dritter Parameter, der die Kraftentwicklung des VMO auf die Patella beeinflusst, ist die kraniokaudale VMO-Ausdehnung in Relation zur Patella.

Die Insertionsstelle der Sehne des VMO befindet sich in der oberen Hälfte der Patella (LIN et al. 2008). Der Ansatzpunkt des VMO hat wiederum Einfluss auf den VMO-Patella-Wirkbereich. Je proximaler der VMO inseriert, desto weniger Zugkraft kann auf die Patella wirken.

Verschiedene Autoren haben in ihren Studien bei Patienten mit PFPS festgestellt, dass der VMO proximaler und abnormal orientiert ist (BOSE et al. 1980, HUBBARD et al. 1997, LIN et al. 2010). KOSKINEN und KUJALA (1992) konnten außerdem in ihrer Untersuchung zeigen, dass sich das Insertionslevel des $M$. vastus medialis signifikant proximaler bei Patienten mit Patellaluxation befand. Daraus ergab sich die Vermutung, dass die mediale Zugkraft des M. quadriceps weniger effektiv sein könnte als bei normalem Ansatzbereich. Sie schlussfolgerten, dass bei Instabilitätsproblematik der $M$. vastus medialis bereits proximal des oberen Patellapols ende und daher die Patella nicht schräg von dorsomedial, sondern vielmehr von proximal erreicht werde und sich dadurch ein veränderter VMO-Winkel sowie ein veränderter Wirkbereich ergebe (KOSKINEN und KUJALA 1992).

In der vorliegenden Studie wurde die kraniokaudale Ausdehnung des VMO in Relation zur Patella gemessen und so auf die VMO-Patella-Kraftwirkung geschlossen. Es konnte kein signifikanter Unterschied zwischen beiden Studiengruppen und der Kontrollgruppe festgestellt werden. In allen Gruppen ergaben sich ähnliche Werte für die kraniokaudale VMO-Ausdehnung. Folglich ergab sich kein Hinweis darauf, dass der VMO bereits vor der Erstluxation einen proximaler gelegenen Insertionsbereich hat bzw. dass sich der Ansatzbereich bei chronischen Luxationspatienten nach proximal verschiebt.

Insgesamt sind die Ergebnisse der Untersuchung der VMO-Parameter mit neueren Studien vereinbar, die in der Therapie des PFPS langsam den Therapieansatz verlassen, die Rehabilitation und Kräftigung des Quadrizeps als zwingende Notwendigkeit für eine erfolgreiche Regeneration zu sehen (PATTYN et al. 2013). Im Einklang dazu steht auch die Aussage von GRABINER et al. (1994), dass der VMO im Gegensatz zu den anderen Komponenten des $M$. quadriceps nicht selektiv und willkürlich aktiviert werden könne und dies somit mit entsprechenden physiotherapeutischen Therapiekonzepten, die auf die selektive VMO-Kräftigung abzielen, kollidiere.

Gleichzeitig ergibt sich hieraus eine Erklärung, weshalb operative Therapieverfahren, die mittels proximaler Weichteil-Rekonstruktion auf die Stärkung des stabilisierenden Effekts des VMO abzielen (OSTERMEIER et al. 2007), relativ häufig fehlschlagen. Mutmaßlich 
adressieren diese nicht die Hauptpathologie der Patellaluxation. OSTERMEIER et al. (2007) haben den Effekt dieser Operationen untersucht und festgestellt, dass diese Verfahren den medialen Anpressdruck der Patella erhöhen. Die Patienten der vorliegenden Studie zeigten anatomische Prädispositionsfaktoren (Trochleadysplasie, Patella alta und erhöhter TT-TG-Abstand) bei gleichzeitig relativ normal konstituierter VMO-Morphologie, sodass der VMO als Ziel der operativen Therapie die falsche Grundlage sein könnte.

Für die Auswertung dieser Studie wurde zwischen akuter Patella-Erstluxation und Rezidivluxationen unterschieden.

Die Patienten der Studiengruppe AE und CL der vorliegenden Untersuchung präsentieren das typische anatomische Risikoprofil für Patellaluxationen mit Patella alta, erhöhtem TT-TG-Abstand und einer Trochleadysplasie. In der Kontrollgruppe fehlen entsprechende Risikomerkmale, sodass sich hier eine signifikante Differenz mit $p<0,0001$ (Trochleadysplasie), $p<0,01$ (TT-TG-Abstand) und $p<0,0053$ (Patellahöhe) ergibt. Im Zusammenhang mit dem Ergebnis des nicht signifikanten Unterschieds in der VMO-Morphologie der Studiengruppe und der Kontrollgruppe deutet dies darauf hin, dass ein augenscheinlich normal konstituierter VMO-Muskel bei Bestehen anderer anatomischer Prädispositionen nicht in der Lage ist, die Stabilität des Patellofemoralgelenks zu erhalten bzw. eine Luxation zu verhindern. Entscheidendes Ergebnis dieser Studie ist daher, dass der VMO vor dem Hintergrund der klinischen und anatomischen Situation der patellofemoralen Instabilität im komplexen Zusammenspiel der verschiedenen Stabilisationsfaktoren nur eine untergeordnete Rolle spielt. Der VMO scheint als Prädispositionsfaktor für Erstluxationen - aber auch für chronische Luxationen - von eingeschränkter Bedeutung zu sein. Einzig der beobachtete Trend zu einem kleineren VMO-Faserwinkel und einer geringeren Querschnittsfläche in der Gruppe der chronischen Luxationspatienten lässt die Annahme zu, dass es eventuell durch die bestehende Instabilität und veränderte körperliche Belastung zur sekundären VMO-Atrophie und posttraumatisch zum veränderten VMO-Faserverlauf gekommen ist. Fraglich ist, ob dies tatsächlich Auswirkungen auf die Rezidivluxationen des Patellofemoralgelenks hat. Sicher ist hingegen, dass für die patellofemorale Stabilität andere Prädispositionsfaktoren eine entscheidendere Bedeutung haben (DEJOUR et al 1994, NIKKU et al. 2009, BALCAREK et al. $2010 \mathrm{~b}$, LEITLINIE Patellaluxation 2014).

Bei der Diskussion der dargestellten Ergebnisse sollten jedoch auch die Grenzen und Schwächen dieser Studie berücksichtigt werden. Zunächst wurden in dieser Arbeit drei morphologische Parameter des VMO bestimmt. Angenommen wurde, dass diese die Konstitution und die Fähigkeit des Muskels repräsentieren und aktiv stabilisierend auf das Patellofemoralgelenk einwirken. Andererseits kann eine VMO-Insuffizienz auch durch eine 
neuromuskuläre Dysfunktion oder durch ein Ungleichgewicht in der Aktivierung des VMO und des VL bedingt sein. Daher ist nicht auszuschließen, dass diese Faktoren für die patellofemorale Instabilität eine Rolle spielen können. Da die Patienten generell nicht ahnen, dass sie eine Patellaluxation erleiden werden, ist es allerdings kaum möglich, diese Faktoren bereits vor der Erstluxation, z.B. mittels Elektromyographie, zu erfassen.

Des Weiteren waren die Patienten der Kontrollgruppe nicht in optimaler gesundheitlicher Verfassung. Die MRT-Untersuchung wurde aufgrund akuter Verletzungen durchgeführt. Dabei wiesen vier Patienten eine Meniskusläsion, 14 Patienten eine vordere und ein Patient eine hintere Kreuzbandruptur auf. Keine relevanten Verletzungen fanden sich bei drei Patienten. Allerdings hat keiner der Kontrollpatienten vor dem entsprechenden Trauma Beschwerden im Kniegelenk, insbesondere nicht im Patellofemoralgelenk, gehabt.

Außerdem konnte in dieser Studie nicht das exakte VMO-Muskelvolumen berechnet werden. Daher wurden die Messungen der VMO-Querschnittsfläche auf drei Höhen bzw. angrenzenden Schichten durchgeführt und die einzelnen Werte summiert. Dieses Vorgehen soll die Dreidimensionalität des Muskels imitieren. Es repräsentiert dabei natürlich trotzdem nicht das genaue Muskelvolumen.

Abschließend ist zu erwähnen, dass die Gruppen in Bezug auf das Geschlecht, den BMI und die physische Aktivität untereinander vergleichbar waren. Das mittlere Alter der Patienten hingegen lag bei 23,9, 19,4 und 21,3 Jahren $(p=0,007)$ in der Kontrollgruppe, der Gruppe AE und CL, und der Unterschied erreichte somit eine statistische Signifikanz. Aufgrund der zufriedenstellenden Übereinstimmung für die Parameter BMI, Geschlecht und physische Aktivität darf jedoch angenommen werden, dass die geringe Altersdifferenz keine relevante Verzerrung der Ergebnisse bewirkt hat. 


\subsection{Fazit}

Diese Arbeit hat sich mit der Untersuchung der Bedeutung des VMO für die Entstehung akuter und chronischer Patellaluxationen beschäftigt. Untersuchungsziel war die Analyse der morphologischen Charakteristiken des VMO im Kontext der lateralen Patellainstabilität. In dieser Studie wurden die Ergebnisse der VMO-Morphologie mit der typischen klinischen und anatomischen Situation eines instabilen Patellofemoralgelenks korreliert.

Es konnten dabei keine signifikanten Unterschiede in der VMO-Morphologie zwischen den Patienten mit akuter Erstluxation, den chronischen Luxationspatienten und der asymptomatischen Kontrollgruppe nachgewiesen werden. Insbesondere die akute Erstluxation ist daher höchstwahrscheinlich nicht vorrangig durch muskuläre Defizite bedingt.

Gleichzeitig lassen diese Ergebnisse vermuten, dass ein normal konstituierter VMO-Muskel demnach auch nicht in der Lage ist, bei Präsenz des typischen Risikoprofils der patellofemoralen Instabilität mit den beschriebenen anatomischen Prädispositionsfaktoren (Patella alta, Trochleadysplasie und erhöhter TT-TG-Abstand) die Stabilität im Patellofemoralgelenk zu erhalten bzw. eine Luxation sogar zu verhindern.

Es ergibt sich als Konsequenz daraus, dass der VMO-Muskel vor dem Hintergrund der klinischen und anatomischen Situation der lateralen Patellainstabilität im komplexen Zusammenspiel der verschiedenen Stabilisatoren des Patellofemoralgelenks eine untergeordnete Rolle zu spielen scheint. Somit ist seine Bedeutung als Prädispositionsfaktor insbesondere für die akute Patella-Erstluxation, aber auch für die Entwicklung von Rezidivluxationen, einzuschränken. 


\section{Zusammenfassung}

Die Luxation der Patella ist ein häufiges Krankheitsbild in der Unfallchirurgie und Orthopädie

und macht ca. 2-3\% aller Knieverletzungen aus. $\mathrm{Da}$ an der Stabilität des Patellofemoralgelenks knöcherne, ligamentäre und muskuläre Strukturen beteiligt sind, gestaltet sich die Ätiopathogenese vielfältig. Zudem ist vor dem Hintergrund des individuellen Instabilitätsrisikos und der Wahl der adäquaten Therapie die genaue Kenntnis der relevanten Stabilitätsfaktoren im Kontext der individuellen Anatomie entscheidend. Trotz vieler Fortschritte in vergangenen Studien bleiben Fragen bezüglich einiger Prädispositionsfaktoren und der entsprechenden Therapie ungeklärt.

In dieser Arbeit wurden die morphologischen Eigenschaften des VMO und seine Bedeutung als prädisponierender Faktor für die patellofemorale Instabilität weiter untersucht. Mit Hilfe von MRT-Schnittbildern wurden die VMO-Parameter (Querschnittsfläche, Muskelfaserwinkel und kraniokaudale Ausdehnung) bei Patienten mit einer patellofemoralen Instabilität bestimmt und mit einer asymptomatischen Kontrollgruppe verglichen. Parallel wurden die Prädispositionsfaktoren Trochleadysplasie, Patella alta und TT-TG-Abstand erhoben.

Insgesamt wurden 82 Patienten (41 Frauen, 41 Männer), die die Einschlusskriterien erfüllten, in die Studie eingeschlossen und in drei Gruppen unterteilt: 30 Patienten mit akuter Patellaerstluxation, 30 Patienten mit chronischer patellofemoraler Instabilität und 22 Kontrollpatienten. Um die untersuchten VMO-Parameter unter den Gruppen vergleichbar zu machen, wurden das Alter, der BMI sowie die körperliche Aktivität mittels des BAECKE-Scores der Patienten erhoben und die Gruppen in Bezug auf diese Parameter angeglichen.

Die Ergebnisse dieser Studie zeigten in Bezug auf die untersuchten VMO-Parameter keine signifikanten Unterschiede zwischen den Patienten mit akuter Erstluxation, den chronischen Luxationspatienten und der asymptomatischen Kontrollgruppe. Lediglich in der Gruppe der chronischen Luxationen konnte ein Trend zur kleineren VMO-Querschnittsfläche beobachtet werden. Die Patienten der Studiengruppen präsentierten außerdem das typische anatomische Risikoprofil für Patellaluxationen mit einer Patella alta, einem erhöhtem TT-TG-Abstand und einer Trochleadysplasie, während in der Kontrollgruppe entsprechende Risikomerkmale fehlten, sodass sich hier eine signifikante Differenz ergab.

Die Ergebnisse lassen vermuten, dass ein normal konstituierter VMO-Muskel demnach wahrscheinlich nicht in der Lage ist, bei Präsenz des typischen Risikoprofils der patellofemoralen Instabilität mit den beschriebenen anatomischen Prädispositionsfaktoren 
(Patella alta, Trochleadysplasie und erhöhter TT-TG-Abstand) die Stabilität im Patellofemoralgelenk zu erhalten bzw. eine Luxation zu verhindern.

Das entscheidende Ergebnis dieser Studie ist daher, dass der VMO vor dem Hintergrund der klinischen und anatomischen Situation der patellofemoralen Instabilität im komplexen Zusammenspiel der verschiedenen Stabilisationsfaktoren nur eine untergeordnete Rolle zu spielen scheint und als Prädispositionsfaktor für Erstluxationen, aber auch für die Entwicklung von Rezidivluxationen von eingeschränkter Bedeutung zu sein scheint. 


\section{Anhang}

\subsection{Abbildungsverzeichnis}

AbBILDUNG 1: Topographische LAGE DES MPFL ZU SEINEN BENACHBARTEN STRUKTUREN (MODIFIZIERT NACH AMIS ET

AL. 2003)

ABBiLdUng 2: MeSSUNG DER VMO-QUERSCHNITTSFLÄCHE. 24

AbBildung 3: MESSUng des VMO-FASERVERLAUFWINKELS 25

AbBildung 4: MesSung der KRANIOKAUdALEN VMO-Ausdehnung In ReLATION ZUR PATELLA. 26

AbBiLdung 5: Messung des TT-TG-ABSTANDES. 29

AbBildung 6: KLASSifiKATION DER TROCHLEADYSPLASIE (MODIFIZIERT NACH DEJOUR ET AL. 1998). 30

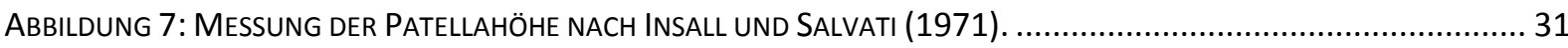

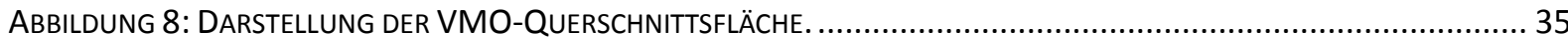

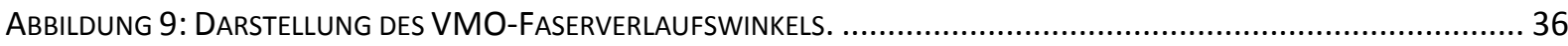

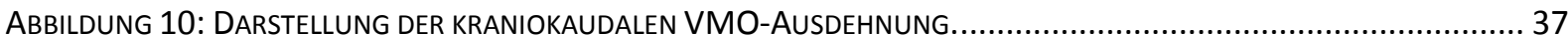

AbBILDUNG 11: DARSTELLUNG DER HÄUfigkeIT DER TROCHLEAdYSPLASIE IN DEN STUDIENGRUPPEN............................. 39

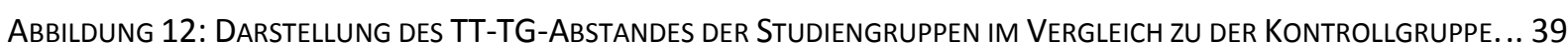
ABbildung 13: DARStellung der PATELLAHÖHE. 40

\subsection{Tabellenverzeichnis}

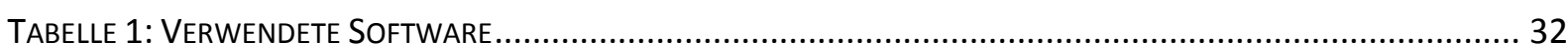

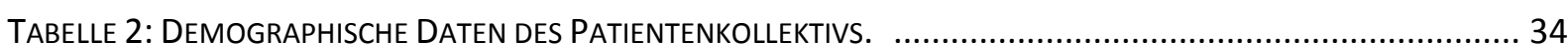

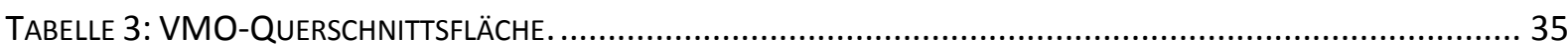

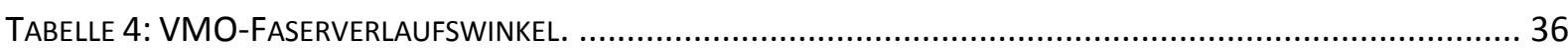

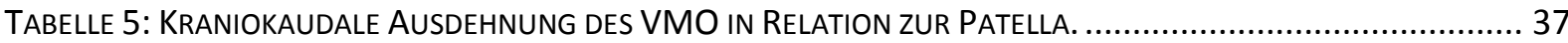

TABELLE 6: VERGLEICH DER ANATOMISCHEN HAUPTPARAMETER TROCHLEADYSPLASIE, TT-TG-ABSTAND UND

PATELLAHÖHE. 38

TABELLE 7: INTRA- UND INTERTESTER-RELIABILITÄT. 41 


\subsection{Abkürzungsverzeichnis}

Abb.

Abbildung

$\mathrm{AE}$

akute Erstluxation

AM

M. adductor magnus

$\mathrm{BMI}$

Body-Mass-Index

bzw.

beziehungsweise

ca.

circa

$95 \% \mathrm{Cl}$

$95 \%$ Konfidenzintervall

CL

chronische Luxation

$\mathrm{cm}^{2}$

Quadratzentimeter

CT

Computertomographie

FW

Faserverlaufswinkel

K

Kontrollgruppe

KkA

kraniokaudale Ausdehnung

Lig.

Ligamentum

M.

Musculus

$\operatorname{Max}$

Maximum

Min

Minimum

$\mathrm{MJ} / \mathrm{h}$

Megajoule / Stunde

$\mathrm{mm}$

Millimeter

MPFL

Lig. patellofemorale mediale

MRT

Magnetresonanztomographie

PACS

Picture Archiving and Communication System

PFG

Patellofemoralgelenk

PFPS

patellofemorales Schmerzsyndrom (patellofemoral pain syndrom)

QF

Querschnittsfläche

S.

siehe

SD

standard deviation (Standardabweichung) 
TT-TG

u.a.

VLO

VML

VMO

vs.

z.B.
Tuberositas tibiae-trochlear groove

unter anderem

M. vastus lateralis

M. vastus medialis longus

M. vastus medialis obliquus

versus

zum Beispiel

\subsection{Baecke-Score Fragebogen}

\section{Fragebogen zur körperlichen Aktivität der Patienten}

1) Was machen Sie beruflich?

2) Bei der Arbeit sitze ich

3) Bei der Arbeit stehe ich

4) Bei der Arbeit gehe ich

5) Bei der Arbeit hebe ich schwere Gegenstände

6) Nach der Arbeit bin ich ... müde.

7) Auf der Arbeit schwitze ich

8) Im Vergleich zu anderen in meinem Alter, denke ich, dass meine Arbeit ... ist.

9) Machen Sie Sport?

Wenn ja:

Welchen Sport üben Sie am häufigsten aus? Wie viele Stunden pro Woche? Wie viele Monate im Jahr?

Wenn 2. Sportart:

Welche Sportart?

Wie viele Stunden pro Woche?

Wie viele Monate im Jahr?

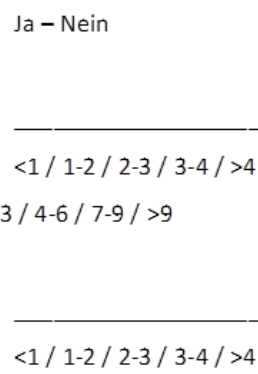

$\mathrm{Ja}-$ Nein

$<1 / 1-2 / 2-3 / 3-4 />4$

$<1 / 1-2 / 2-3 / 3-4 />4$

$1-3-5$

$1-2-3-4-5$

$1-2-3-4-5$

$1-2-3-4-5$

$1-2-3-4-5$

$5-4-3-2-1$

$5-4-3-2-1$

$5-4-3-2-1$ $<1 / 1-3 / 4-6 / 7-9 />9$ $<1 / 1-3 / 4-6 / 7-9 />9$
Intensität:

Zeit:

Anteil:

$0.5-1.5-2.5-3.5-4.5$

Intensität: $\quad 0.76-1.26-1.76$

Zeit: $\quad 0.5-1.5-2.5-3.5-4.5$

Anteil:

10) Im Vergleich zu anderen in meinem Alter, denke ich, dass die sportliche Aktivität in meiner Freizeit ... ist.

Viel mehr - mehr - gleich - weniger - viel weniger

$5-4-3-2-1$

11) Während meiner Freizeit schwitze ich ...

12) In meiner Freizeit mache ich ... Sport.

sehr oft - oft - manchmal - selten - nie

$5-4-3-2-1$

Nie - selten - manchmal - oft - sehr oft

$1-2-3-4-5$

13) In meiner Freizeit gucke ich ... Fernsehen

Nie - selten - manchmal - oft - sehr oft

$1-2-3-4-5$

14) In meiner Freizeit gehe ich ... spazieren.

Nie - selten - manchmal - oft - sehr oft

$1-2-3-4-5$

15) In meiner Freizeit fahre ich ... Fahrrad.

Nie - selten - manchmal - oft - sehr oft

$1-2-3-4-5$

16) Wie viele Minuten am Tag gehen und/ ode fahren Sie Fahrrad (zur Arbeit, Schule oder zum Einkaufen)? 


\section{Literaturverzeichnis}

Ahmad CS, Stein BE, Matuz D, Henry JH (2000): Immediate surgical repair of the medial patellar stabilisers for acute patellar dislocation. A review of eight cases. Am J Sports Med. $\underline{28}$, 804-810

Amis AA, Firer P, Mountney J, Senavongse W, Thomas NP (2003): Anatomy and biomechanics of the medial patellofemoral ligament. The Knee $\underline{10}, 215-220$

Amis AA, Senavongse W, Bull AM (2006): Patellofemoral kinematics during knee flexionextension: an in vitro study. J Orthop Res $\underline{24}, 2201-2211$

Arendt EA, Fithian DC, Cohen E (2002): Current concepts of lateral patella dislocation. Clin Sports Med 21, 499-519

Atkin DM, Fithian DC, Marangi KS, Stone ML, Dobson BE, Mendelsohn C (2000): Characteristics of patients with primary acute lateral patellar dislocation and their recovery within the first 6 months of injury. Am J Sports Med 28, 472-479

Bachmann M und Pagenstert G (2010): Arthroskopische Biomechanik bei patellofemoralen Beschwerden. Arthroskopie 23, 170-175

Baecke JA, Burema J, Frijters JE (1982): A short questionnaire for the measurement of habitual physical activity in epidemiological studies. Am J Clin Nutr $\underline{36}, 936-942$

Balcarek P und Frosch K-H (2012): Die Patellaluxation im Kindes- und Jugendalter. Arthroskopie 25, 266-274

Balcarek P, Jung K, Ammon J, Walde TA, Frosch S, Schüttrumpf JP, Stürmer KM, Frosch K-H (2010 a). Anatomy of lateral patellar instability: trochlear dysplasia and tibial tubercle-trochlear groove distance is more pronounced in women who dislocate the patella. Am J Sports Med $\underline{38}, 2320-2327$

Balcarek P, Ammon J, Frosch S, Walde TA, Schüttrumpf JP, Ferlemann KG, Lill H, Stürmer $\mathrm{KM}$, Frosch K-H (2010 b): Magnetic resonance imaging characteristics of the medial patellofemoral ligament lesion in acute lateral patellar dislocations considering trochlear dysplasia, patella alta, and tibial tuberosity-trochlear groove distance. Arthroscopy 26, 926-935

Balcarek P, Walde TA, Frosch S, Schüttrumpf JP, Wachowski MM, Stürmer KM, Frosch $\mathrm{K}-\mathrm{H}$ (2011): Patellar dislocations in children, adolescent and adults: A comparative MRI study of medial patellofemoral ligament injury patterns and trochlear groove anatomy. Eur J Radiol $\underline{79}$, 415-420 
Bamman MM, Newcomer BR, Larson-Meyer DE, Weinsier RL, Hunter GR (2000):

Evaluation of the strength-size relationship in vivo using various muscle size indices. Med Sci Sports Exerc 32, 1307-1313

Bennet WF, Doherty N, Hallisey MJ (1993): Insertion orientation of terminal vastus lateralis obliquus and vastus medialis obliquus muscle fibers in human knees. Clin Anat $\underline{6}$, 129-134

Berry PA, Teichtahl AJ, Galevska-Dimitrovska A, Hanna FS, Wluka AE, Wang Y, Urquhart DM, English DR, Giles GG, Cicuttini FM (2008): Vastus medialis cross-sectional area is positively associated with patella cartilage and bone volumes in a pain-free community-based population. Arthritis Res Ther $\underline{10}, 143$

Boden BP, Pearsall AW, Garrett WJ Jr, Feagin JJ Jr (1997): Patellofemoral Instability: Evaluation and Management. J Am Acad Orthop Surg $\underline{5}$, 47-57

Bose K, Kanagasuntheran R, Osman MB (1980): Vastus medialis oblique: an anatomic and physiologic study. Orthopedics $\underline{3}, 880-883$

Bull AM und Amis AA: Biomechanik. In Orthopädie und orthopädische Chirurgie: Knie. 67 Tabellen; hrsg. v. Kohn D u.a.; Thieme Verlag, Stuttgart 2005, 35-51

Bull AM, Katchburian M, Shih Y, Amis AA (2002): Standardisation of the description of patellofemoral motion and comparison between different techniques. Knee Surg Sports Traumatol Arthrosc $\underline{10}, 184-193$

Busch MT: Sports medicine in children and adolescents; in: Lovell and Winter's pediatric orthopedics, $5^{\text {th }}$ ed., Vol. II; hrsg. v. Morrissy RT, Weinstein SL u.a.; Williams \& Wilkins, Baltimore, 2001, 1306-1308

Cohn SH, Vartsk D, Yasumura S, Sawitsky A, Zanzi I, Vaswani A, Ellis KJ (1980): Compartmental body composition based on total-body nitrogen, potassium, and calcium. Am J Physiol 239, 524-530

Conlan T, Garth WP, Lemons JE (1993): Evaluation of the medial soft-tissue restraints of the extensor mechanism of the knee. J Bone Joint Surg Am $\underline{75}, 682-693$

Dejour D, Reynaud P, Lecoultre B (1998): Douleurs et instabilité rotulienne. Essai de classification. Méd et Hyg $\underline{56}, 1466-1471$

Dejour H, Walch G, Nove-Josserand L, Guier C (1994): Factors of patellar instability: an anatomic radiographic study. Knee Surg Sports Traumatol Arthrosc 2

Desio SM, Burks RT, Bachus KN (1998): Soft Tissue Restraints to Lateral Patellar Translation in the Human Knee. Am J Sports Med 26, 59-65 
Devereaux MD und Lachmann SM (1984): Patello-femoral arthralgia in athletes attending a Sports Injury Clinic. Br J Sports Med $\underline{18}, 18-21$

Diehl L und Garrett WE: Acute dislocation of the patella in the adult. In Delee and Drez's Orthopaedic Sports Medicine, 2nd ed.; hrsg. v. Delee J, Drez D, Miller M u.a.; Saunders, Philadelphia 2003, 1697-1710

Doucette SA und Goble EM (1992): The effect of exercise on patellar tracking in lateral patellar compression syndrome. Am J Sports Med 20, 434-440

Elias DA und White LM (2004): Imaging of patellofemoral disorders. Clin Radiol $\underline{59}, 543-$ 557

Elias DA, White LM, Fithian DC (2002): Acute lateral patellar dislocation at MR imaging: Injury patterns of medial patellar soft-tissue restraints and osteochondral injuries of the inferomedial patella. Radiology $\underline{225}, 736-743$

Farahmand F, Senavongse W, Amis AA (1998): Quantitative study of the quadriceps muscles and trochlear groove geometry related to instability of the patellofemoral joint. J Orthop Res $\underline{16}, 136-143$

Fahramand F, Naghi Tahmasbi M, Amis A (2004): The contribution of the medial retinaculum and quadriceps muscles to patellar lateral stability - an in vitro study. Knee 11, 89-94

Fink C, Hoser C, Benedetto KP (1993): Sports capacity after rupture of the anterior cruciate ligament--surgical versus non-surgical therapy. Akt Traumatol 23, 371-375

Fithian DC, Paxton EW, Stone ML, Silva P, Davis DK, Elias DA, White LM (2004): Epidemiology and natural history of acute patellar dislocation. Am J Sports Med $\underline{32}$, 1114-1121

Fox T (1975): Dysplasia of the quadriceps mechanism: hypoplasia of the vastus medialis muscle as related to the hypermobile patella syndrome. Surg Clin North Am $\underline{55}$, $199-226$

Frontera WR, Hughes VA, Lutz KJ, Evans WJ (1991): A cross-sectional study of muscle strength and mass in 45- to 78-yr-old men and women. J Appl Physiol 71, 644-650

Fulkerson JP: Patellar subluxation; in: Disorders of the Patellofemoral Joint; $4^{\text {th }}$ ed.; hrsg. v. Fulkerson JP; Lippincott Williams \& Wilkins, Philadelphia, 2004, 185

Gardner E und O'Rahilly R (1968): The early development of the knee joint in staged human embryos. J Anat 102, 289-299 
Gilleard W, McConnell J, Parsons D (1998): The effect of patellar taping on the onset of vastus medialis obliquus and vastus lateralis muscle activity in persons with patellofemoral pain. Phys Ther $\underline{78}, 25-32$

Glard Y, Jouve JL, Panuel M, Adalian P, Tardieu C, Bollini G (2005): An anatomical and biometrical study of the femoral trochlear groove in the human fetus. J Anat 206, 411-413

Goh JC, Lee PY, Bose K (1995): A cadaver study of the function of the oblique part of vastus medialis. J Bone Joint Surg $\mathrm{Br} \underline{77}, 225-231$

Goodfellow J, Hungerford DS, Zindel M (1976): Patellofemoral joint mechanics and pathology. J Bone Joint Surg $\underline{58}, 291-299$

Grabiner MD, Koh TJ, Draganich LF (1994): Neuromechanics of the patellofemoral joint. Med Sci Sports Exerc 26, 10-21

Hawkins RJ, Bell RH, Anisette G (1986): Acute patellar dislocations. The natural history. Am J Sports Med 14, 117-120

Hensler D und Schöttle PB (2010): Erweiterte Indikationen für die Rekonstruktion des medialen patellofemoralen Ligaments. Arthroskopie $\underline{23}$, 184-190

Hing CB, Smith TO, Donell S, Song F (2011): Surgical versus non-surgical interventions for treating patellar dislocation. Cochrane Database Syst Rev $\underline{9}$, (11)

Hinton RY und Sharma KM (2003): Acute and recurrent patellar instability in the young athlete. Orthop Clin North Am $\underline{34}$, 385-396

Hubbard JK, Sampson HW, Elledge JR (1997): Prevalence and morphology of the vastus medialis oblique muscle in human cadavers. Anat Rec $\underline{249}$, 135-142

Huberti HH, Hayes WC, Stone JL, Shybut GT (1984): Force ratios in the quadriceps tendon and ligamentum patellae. J Orthop Res 2, 49-54

Insall J und Salvati E (1971): Patella position in the normal knee joint. Radiology $\underline{101}$, 101-104

Jan MH, Lin DH, Lin JJ, Lin CH, Cheng CK, Lin YF (2009): Differences in sonographic characteristics of the vastus medialis obliquus between patients with patellofemoral pain syndrome and healthy adults. Am J Sports Med 37, 1743-1749

Kaufer H (1971): Mechanical function of the patella. J Bone Joint Surg Am $\underline{53}, 1551-1560$

Kaya D, Citaker S, Kerimoglu U, Atay OA, Nyland J, Callaghan M, Yakut Y, Yüksel Y, Doral MN (2011): Women with patellofemoral pain syndrome have quadriceps femoris volume and strength deficiency. Knee Surg Sports Traumatol Arthrosc 19, 242-247 
Koeter S, Diks MJ, Anderson PG, Wymenga AB (2007): A modified tibial tubercle osteotomy for patellar maltracking: results at two years. J Bone Joint $\mathrm{Surg} \mathrm{Br} \underline{89}$, 180-185

Koskinen SK und Kujala UM (1992): Patellofemoral Relationships and Distal Insertion of the Vastus Medialis Muscle: A Magnetic Resonance Imaging Study in Nonsymptomatic Subjects and in Patients with Patellar Dislocation. Arthroscopy $\underline{8}$, 465-468

Leitlinie Patellaluxation. Jagodzinski M, Niemeyer P, Zeichen J, Balcarek P (2014): S1Leitlinie 012/024: Patellaluxation. http://www.awmf.org/uploads/tx_szleitlinien/012024I_S1_Patellaluxation_2014-06.pdf

Leroux A, Belanger M, Boucher JP (1995): Pain effect on monosynaptic and polysynaptic reflex inhibition. Arch Phys Med Rehabil 76, 576-582

Lieb FJ und Perry J (1968): Quadriceps function. An anatomical and mechanical study using amputated limbs. J Bone Joint Surg $\underline{50}, 1535-1548$

Lin F, Wilson NA, Makhsous M, Press JM, Koh JL, Nuber GW, Zhang LQ (2010): In vivo patellar tracking induced by individual quadriceps components in individuals with patellofemoral pain. J Biomech $\underline{43}, 235-241$

Lin YF, Lin JJ, Jan MH, Wei TC, Shih HY, Cheng CK (2008): Role of the vastus medialis obliquus in repositioning the patella - a dynamic computed tomography study. Am J Sports Med $\underline{36}, 741-746$

Lippacher S, Dejour D, Elsharkawi M, Dornacher D, Ring C, Dreyhaupt J, Reichel H, Nelitz M (2012): Observer agreement on the Dejour trochlear dysplasia classification: a comparison of true lateral radiographs and axial magnetic resonance images. Am J Sports Med 40, 837-843

Mariani PP und Caruso I (1979): An electromyographic investigation of subluxation of the patella. J Bone Joint Surg $\mathrm{Br} \underline{61}, 169-171$

Merican AM, Sanghavi S, Iranpour F, Amis AA (2009): The structural properties of the lateral retinaculum and capsular. J Biomech $\underline{42}, 2323-2329$

Mitsiopoulos N, Baumgartner RN, Heymsfield SB, Lyons W, Gallagher D, Ross R (1998): Cadaver validation of skeletal muscle measurement by magnetic resonance imaging and computerized tomography. J Appl Physiol 도, 115-122

Mochizuki T, Nimura A, Tateishi T, Yamaguchi K, Muneta T, Akita K (2013): Anatomic study of the attachment of the medial patellofemoral ligament and its characteristic 
relationships to the vastus intermedius. Knee Surg Sports Traumatol Arthrosc 21, 305-310

Mutschler W: Anatomie und Biomechanik des Kniegelenks. In: Das Knie: Verletzungen, Verletzungsfolgen, Erkrankungen. Band I; hrsg. v. Burri C, Mutschler W u.a.; Hippokrates Verlag Stuttgart 1982, 10-19

Nietosvaara Y (1994): The femoral sulcus in children. An ultrasonographic study. J Bone Joint Surg $\underline{76}$, 807-809

Nikku R, Nietosvaara Y, Aalto K, Kallio PE, Michelsson JE (1997): Operative versus closed treatment of primary dislocation of the patella. Similar 2-year results in 125 randomized patients. Acta Orthop Scand $\underline{68}, 419-423$

Nikku R, Nietosvaara Y, Aalto K, Kallio PE (2005): Operative treatment of primary patellar dislocation does not improve medium-term outcome: A 7-year follow-up report and risk analysis of 127 randomized patients. Acta Orthop $\underline{76}, 699-704$

Nikku R, Nietosvaara Y, Aalto K, Kallio P (2009): The mechanism of primary patellar dislocation. Trauma history of 126 patients. Acta Orthop $\underline{80}, 432-434$

Nomura $E$ (1999): Classification of lesions of the medial patello-femoral ligament in patellar dislocations. Int Orthop 르, 260-263.

Nomura E, Inoue M, Osada N (2005): Anatomical analysis of the medial patellofemoral ligament of the knee, especially the femoral attachment. Knee Surg Sports Traumatol Arthrosc $\underline{13}$, 510-515

Olk A und Hennig F (2002): Anatomische Voraussetzungen der Patellaluxation. Trauma und Berufskrankh 4, 201-205

Ostermeier S und Becher C (2010). Einfluss des Tuberositastransfers auf das patellofemorale Gelenk. Arthroskopie 3, S. 208-213.

Ostermeier S, Holst M, Bohnsack M, Hurschler C, Stukenborg-Colsman C, Wirth CJ (2007): In vitro measurement of patellar kinematics following recontruction of the medial patellofemoral ligament. Knee Surg Sports Traumatol Arthrosc 15, 276-285

Pal S, Besier TF, Draper CE, Fredericson M, Gold GE, Beaupre G, Delp SL (2012): Patellar tilt correlates with vastus lateralis: vastus medialis activation ratio in maltracking patellofemoral pain patients. J Orthop Res $\underline{30,}$ 927-933

Palmu S, Kallio PE, Donell ST, Helenius I, Nietosvaara Y (2008): Acute patellar dislocation in children and adolescents: a randomized clinical trial. J Bone Joint Surg $\underline{90}$, 463470 
Pattyn E, Verdonk P, Steyaert A, Vanden Bossche L, Van den Broecke W, Thijs Y, Witvrouw E (2011): Vastus Medialis Obliquus Atrophy: Does it exist in Patellofemoral Pain Syndrome? Am J Sports Med 39, 1450-1455

Pattyn E, Verdonk P, Steyaert A, Van Tiggelen D, Witvrouw E (2013): Muscle functional MRI to evaluate quadriceps dysfunction in patellofemoral pain. Med Sci Sports Exerc 45, 1023-1029

Pfirrmann CW, Zanetti M, Romero J, Hodler J (2000): Femoral trochlear dysplasia: MR findings. Radiology $\underline{216}$, 858-864

Philippot R, Boyer B, Testa R, Farizon F, Moyen B (2012): The role of the medial ligamentous structures on patellar tracking during knee flexion. Knee Surg Sports Traumatol Arthrosc 므, 331-336.

Powers CM, Landel R, Perry J (1996): Timing and intensity of vastus muscle activity during functional activities in subjects with and without patellofemoral pain. Phys Ther $\underline{76}$, 946-955

Raimondo RA, Ahmad CS, Blankevoort L, April EW, Grelsamer RP, Henry JH (1998): Patellar stabilisation: a quantitative evaluation of the vastus medialis obliquus muscle. Orthopedics $\underline{21}, 791-795$

Rhee SJ, Pavlou G, Oakley J, Barlow D, Haddad F (2012): Modern management of patellar instability. Int Orthop $\underline{36}, 2447-2456$

Sakai N, Luo ZP, Rand JA, An KN (2000): The influence of weakness in the vastus medialis oblique muscle on the patellofemoral joint: an in vitro biomechanical study. Clin Biomech 15, 335-339

Sallay PI, Poggi J, Speer KP, Garrett WE (1996): Acute dislocation of the patella. A correlative pathoanatomic study. Am J Sports Med 24, 52-60

Schoettle PB, Zanetti M, Seifert B, Pfirrmann CW, Fucentese SF und Romero J (2006): The tibial tuberosity-trochlear groove distance; a comparative study between CT and MRI scanning. Knee $\underline{13}$, 26-31

Schöttle P, Beitzel K, Imhoff A (2009): Die kindliche Patellaluxation. Anatomie, Pathomorphologie und Behandlungsstrategien. Arthroskopie 22, 51-59

Senavongse W und Amis AA (2005): The effects of articular, retinacular or muscular deficiencies on patellofemoral joint stability: A biomechanical study in vitro. J Bone Joint Surg Br $\underline{87}, 577-582$ 
Senavongse W, Farahmand F, Jones J, Andersen H, Bull AM, Amis AA (2003):

Quantitative measurement of patellofemoral joint stability: Force-displacement behavior of the human patella in vitro. J Orthop Res $\underline{24}, 780-786$

Strobl W und Grill F (1998): Die Patellaluxation. Orthopäde 27, 197-205

Tuxøe JI, Teir M, Winge S, Nielsen PL (2002): The medial patellofemoral ligament: a dissection study. Knee Surg Sports Traumatol Arthrosc 10, 138-140

Vandervoort AA und McComas AJ (1986): Contractile changes in opposing muscles of the human ankle joint with aging. J Appl Physiol $\underline{61}, 361-370$

Vavken P, Wimmer MD, Camathias C, Quidde J, Valderrabano V, Pagenstert G ( 2013): Treating patella instability in skeletally immature patients. Arthroscopy $\underline{29}, 1410-$ 1422

Walsh W: Recurrent Dislocation of the Knee in the Adult. In: Delee and Drez's Orthopaedic Sports Medicine, 2nd ed.; hrsg. v. Delee J, Drez D, Miller M u.a.; Saunders, Philadelphia 2003, 1710-1749

Warren LF und Marshall JL (1979): The supporting structures and layers on the medial side of the knee: an anatomical analysis. J Bone Joint Surg Am 61, 56-62

White BJ und Sherman OH (2009): Patellofemoral Instability. Bull NYU Hosp Jt Dis. $\underline{67}$, 22-29 


\section{Lebenslauf}

Ich, Swantje Oberthür, wurde am 9. Dezember 1986 als Tochter der Lehrerin Rosemarie Hoberg-Oberthür und des Arztes Dr. Bernd Oberthür in Wilhelmshaven geboren.

Im Sommer 1993 wurde ich eingeschult und besuchte die Grundschule Neuengroden, die Franziskusschule (Orientierungsstufe) sowie die Cäcilienschule, ein staatlich anerkanntes freies Gymnasium in kirchlicher Trägerschaft. Im Jahr 2006 schloss ich die schulische Ausbildung mit dem Abitur und der Note 1,6 ab.

Während der Schulzeit engagierte ich mich mit viel Freude in der Schülervertretung der Cäcilienschule. In der Zeit von 2004-2006 konnte ich mich als Schulsprecherin für die Schulgemeinschaft einsetzen.

Nach meiner Schulzeit entschloss ich mich, zur Verbesserung meiner Sprachkenntnisse für drei Monate ins englischsprachige Ausland zu gehen. In der Zeit von September bis Dezember 2006 absolvierte ich zunächst einen vierwöchigen Sprachkurs in Sydney, Australien. Anschließend hatte ich die Möglichkeit, in einem zweimonatigen Krankenpflegepraktikum am Canada Bay Private Hospital in Sydney das australische Gesundheitssystem kennen zu lernen

Im Wintersemester 2007/2008 begann ich mein Studium der Humanmedizin an der Georg-August-Universität Göttingen. Den 1. Abschnitt der Ärztlichen Prüfung schloss ich im Sommer 2009 ab.

Im Sommer 2010 begann ich in der unfallchirurgischen Abteilung der Universitätsklinik meine Dissertation. Im Rahmen der unfallchirurgischen Kniesprechstunde, an der ich regelmäßig teilnahm, untersuchte ich in einer klinischen Studie das Krankheitsbild der Patellaluxation.

Während der Semesterferien absolvierte ich Famulaturen in den Abteilungen für Anästhesiologie, Gynäkologie und vor allem Unfallchirurgie.

Im Herbst 2013 schloss ich den 2. Abschnitt der Ärztlichen Prüfung an der GeorgAugust-Universität Göttingen ab.

Im Februar 2014 habe ich begonnen, in der Abteilung für Unfallchirurgie der Universitätsmedizin Göttingen als Assistenzärztin zu arbeiten.

Swantje Oberthür 